\title{
A foundation for probabilistic beliefs with or without atoms
}

Citation for published version (APA):

Mackenzie, A. (2018). A foundation for probabilistic beliefs with or without atoms. Maastricht University, Graduate School of Business and Economics. GSBE Research Memoranda No. 013 https://doi.org/10.26481/umagsb.2018013

Document status and date:

Published: 08/05/2018

DOI:

10.26481/umagsb.2018013

Document Version:

Publisher's PDF, also known as Version of record

\section{Please check the document version of this publication:}

- A submitted manuscript is the version of the article upon submission and before peer-review. There can be important differences between the submitted version and the official published version of record.

People interested in the research are advised to contact the author for the final version of the publication, or visit the DOI to the publisher's website.

- The final author version and the galley proof are versions of the publication after peer review.

- The final published version features the final layout of the paper including the volume, issue and page numbers.

Link to publication

\footnotetext{
General rights rights.

- You may freely distribute the URL identifying the publication in the public portal. please follow below link for the End User Agreement:

www.umlib.nl/taverne-license

Take down policy

If you believe that this document breaches copyright please contact us at:

repository@maastrichtuniversity.nl

providing details and we will investigate your claim.
}

Copyright and moral rights for the publications made accessible in the public portal are retained by the authors and/or other copyright owners and it is a condition of accessing publications that users recognise and abide by the legal requirements associated with these

- Users may download and print one copy of any publication from the public portal for the purpose of private study or research.

- You may not further distribute the material or use it for any profit-making activity or commercial gain

If the publication is distributed under the terms of Article $25 \mathrm{fa}$ of the Dutch Copyright Act, indicated by the "Taverne" license above, 


\section{Maastricht University}

Andrew Mackenzie

A foundation for probabilistic beliefs with or without atoms

RM/18/013

\section{GSBE}

Maastricht University School of Business and Economics

Graduate School of Business and Economics

P.O Box 616

NL- 6200 MD Maastricht

The Netherlands 


\title{
A FOUNDATION FOR PROBABILISTIC BELIEFS WITH OR WITHOUT ATOMS
}

\author{
ANDREW MACKENZIE* \\ This draft: April 2, 2017
}

\begin{abstract}
We provide sufficient conditions for a qualitative probability (Bernstein, 1917; de Finetti, 1937; Koopman, 1940; Savage, 1954) that satisfies monotone continuity (Villegas, 1964; Arrow, 1970) to have a unique countably additive measure representation, generalizing Villegas (1964) to allow atoms. Unlike previous contributions, we do so without a cancellation or solvability axiom.

First, we establish that when atoms contain singleton cores, unlikely cores - the requirement that the union of all cores is not more likely than its complement - is sufficient (Theorem 3). Second, we establish that strict third-order atom-swarmingthe requirement that for each atom $A$, the less-likely non-null events are (in an ordinal sense) more than three times as likely as $A$-is also sufficient (Theorem 5). This latter result applies to intertemporal preferences over streams of indivisible objects.
\end{abstract}

Keywords: beliefs, qualitative probability, atoms, atom-swarming.

\section{Introduction}

\section{$1.1 \quad$ Executive summary}

From the doctor's choice of treatment, to the employer's choice of job applicant, to the investor's choice of portfolio, to the mortal's choice of religion, and beyond: much behavior, including much of the economic behavior we observe and strive to model, is the selection of an action with uncertain consequences. Our standard model is founded on the postulate that such choices, when made by someone who is rational, can be decomposed into (1) beliefs about the relative likelihood of events, and (2) tastes among outcomes (Ramsey, 1931). This article revisits a classic question: when are such beliefs consistent with standard probability theory? ${ }^{1}$

*I thank David Ahn, Paulo Barelli, Marcus Berliant, David Dillenberger, Georgios Gerasimou, Itzhak Gilboa, Srihari Govindan, Edward Green, Faruk Gul, Vilmos Komornik, Igor Kopylov, Massimo Marinacci, Kemal Ozbek, Jacob Sagi, Oscar Sheynin, Tomasz Strzalecki, William Thomson, Peter Wakker, Chih-Chun Yang, participants at the 2016 Canadian Economic Theory Conference and the 2016 RUD (Research, Uncertainty, and Decision) Conference, and especially Asen Kochov, Ran Spiegler, and two anonymous referees.

${ }^{1}$ Machina and Schmeidler (1992) call this question the first of two lines of inquiry culminating in the modern theory of subjective probability. 
More precisely, suppose we are given a nonempty set of states $S$, a $\sigma$-algebra of events $\mathcal{A} \subseteq 2^{S}$ with $S \in \mathcal{A}$, and a qualitative probability $\succsim$ on $\mathcal{A}$ : a binary relation on $\mathcal{A}$, consisting of comparisons of events on the basis of relative likelihood, satisfying minimal probabilistic requirements (Bernstein, 1917; de Finetti, 1937; Koopman, 1940; Savage, 1954). When does $\succsim$ admit representation by a $\sigma$-measure ${ }^{2} \mu: \mathcal{A} \rightarrow[0,1]$ ?

A necessary condition is monotone continuity (Villegas, 1964; Arrow, 1970): if $B_{1} \supseteq$ $B_{2} \supseteq \ldots$, and for each $i \in N, B_{i} \succsim A$, then $\cap B_{i} \succsim A{ }^{3}$ On the appeal of this axiom, Arrow writes: "The assumption of Monotone Continuity seems, I believe correctly, to be the harmless simplification almost inevitable in the formalization of any real-life problem." While this continuity axiom is not sufficient on its own (Kraft, Pratt, and Seidenberg, 1959), we obtain sufficient conditions when it is paired with the requirement that there are no atoms: non-null events for which each subset is either equally-likely or null (Villegas, 1964).

Though the practice of using $\sigma$-measures to represent beliefs with atoms is prevalent throughout economic analysis (particularly when the state space is discrete), atoms present non-trivial technical challenges for our problem. In fact, the large literature dedicated to our problem can be classified according to which of the following is imposed:

- there are no atoms;

- the qualitative probability satisfies a "cancellation" axiom;

- the qualitative probability satisfies a "solvability" axiom; or

- there are additional primitives beyond $S, \mathcal{A}$, and $\succsim$.

The contribution of this article is to proceed without imposing any of the above.

First, we show that under monotone continuity, if each atom contains a core - a singleton atom - and if moreover the union of all cores is at most as likely as its complement, then unique $\sigma$-measure representation is guaranteed (Theorem 3 ). In this case, there is an infinitely-divisible event whose probability is at least one-half.

What if each infinitely-divisible event is less likely than its complement? In particular, what if there are no infinitely-divisible events at all? To address this case, we propose strict third-order atom-swarming $\left(3^{+}-A S\right)$ : if there are any atoms at all, then each is sufficiently overwhelmed by less-likely non-null events. ${ }^{4}$ Formally, for each atom $A$, there is a pairwise-disjoint collection of less-likely non-null events that can be partitioned into three $^{5}$ subcollections, with the union of each more likely than $A$ (Figure 1). Our main result is that monotone continuity and $3^{+}-A S$ are sufficient to guarantee $\sigma$-measure representation.

\footnotetext{
${ }^{2}$ In this article, a measure is a finitely-additive probability measure, $\sigma$-additivity is countable-additivity, and a $\sigma$-measure is a $\sigma$-additive measure.

${ }^{3}$ Villegas (1964) and Arrow (1970) give different statements that are logically equivalent; I owe this particularly elegant statement to a referee.

${ }^{4}$ Earlier versions of this article further required that these non-null events were in fact atoms, ruling out the possibility of a finite collection of cores. I am indebted to a referee for suggesting this generalization.

${ }^{5}$ Why three? Why not the analogous $1^{+}-A S$ or $2^{+}-A S$ ? This is addressed over the course of our proof's explanation. For now, we remark only that at least three has appeared in earlier research on a similar topic: given an ordering over a product space, if there are only two "essential" factors, an additional condition is used to guarantee an additive representation (Debreu, 1959). For rough intuition: while addition is a binary operation, its cancellation property is articulated with three elements.
} 


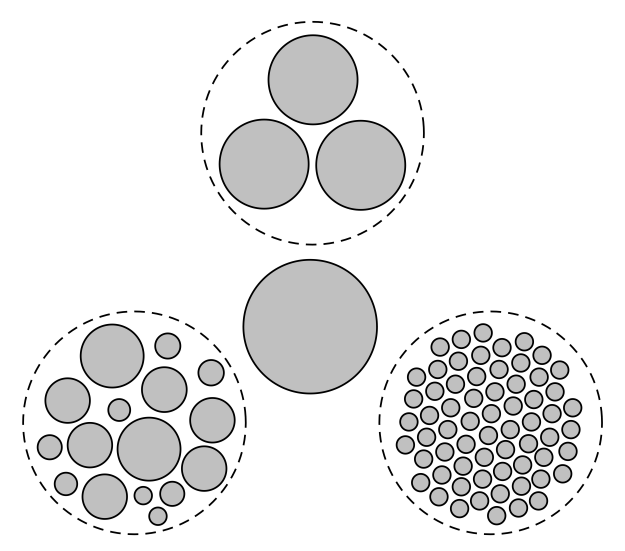

Figure 1: Strict third-order atom-swarming. In the illustration, each gray circle is a non-null event, and a more likely event has a greater area. The center event is an atom, and the smaller events are pairwise-disjoint. Each dashed circle shows a collection of these smaller events whose union is more likely than the center atom; in this way, the atom is 'sufficiently swarmed' by the smaller events. The top collection includes a few relatively large events, the rightmost collection includes many relatively small events, and the leftmost collection includes events of different sizes. Not pictured, but allowed, is an infinite collection of events. The axiom requires that there are three such collections for each atom.

\section{$1.2 \quad$ Related literature}

According to the historian of probability Oscar Sheynin (Sheynin, 2005), Boole was the first to call for an axiomatic foundation for probability, ${ }^{6}$ and this call was prominently reiterated over four decades later in Hilbert's Sixth Problem. ${ }^{7}$ It would be another three decades before Kolmogorov provided today's standard axioms for cardinal probability, explaining that this task was "a rather hopeless one before the introduction of Lebesgue's theories of measure and integration" (Kolmogoroff, 1933). Kolmogorov's axioms involve $\sigma$-algebras, which are appropriately a subclass of the logical algebras studied by Boole: anticipating Kolmogorov, Boole wrote that "the theory of probabilities is coextensive with that of logic" because "as simple events are expressed by simple propositions, so combinations of events are expressed by compound propositions" (Boole, 1851). ${ }^{8}$

${ }^{6}$ From Boole (1854): "It cannot, I think, be doubtful that the theory of probabilities belongs to that class of sciences which are termed pure sciences[...]From this idea, from the definition of the measure of probability by which it becomes associated with a number, and from the laws of thought with which it is connected through its having to do with events capable of logical expression, flow the axioms and first principles of the science[...]I go on to observe, that such principles, if truly axiomatic, lead in every pure science, and therefore in the theory of probabilities, to a developed system of truth, or of methods for the attainment of truth, which possess certain invariable characteristics never found unimpaired where error has been permitted to enter."

${ }^{7}$ From Hilbert (1900): "The investigations on the foundations of geometry suggest the problem: to treat in the same manner, by means of axioms, those physical sciences in which mathematics plays an important part; in the first rank are the theory of probabilities and mechanics." The investigations Hilbert refers to constitute the earliest axiomatic work in science that survives to this day (Euclid, circa $300 \mathrm{BC})$.

${ }^{8}$ Indeed, the modern notion of an abstract Boolean algebra (Halmos, 1963) involves 'propositions' that, by Stone's Representation Theorem, can be viewed as events of a state space without loss of generality (Birkhoff, 1935; Stone, 1936). This is not true for $\sigma$-algebras; the lost generality is made explicit by the Loomis-Sikorski Representation Theorem (Loomis, 1947; Sikorski, 1960). Interestingly, 
This intimate relationship between logic and probability does not, however, require a cardinal concept. Indeed, while a cardinal concept is natural for a frequentist interpretation of probability, in which "a probability is the relative frequency of some kind of event in a certain type of sequence of events or, according to some, in a set of events" (Savage, 1961), this interpretation is rejected by Bayesian statisticians, who advocate reaction to evidence using Bayes' Theorem (Bayes, 1763). ${ }^{9}$

An alternative to frequentist probability, favored by many Bayesians, is a subjective (or personal) interpretation of probability (Ramsey, 1931), and perhaps the most natural concept for this interpretation is not cardinal, but ordinal: when confronted with two logical propositions whose truth is not known, one is able to compare the two, as though using a balance, to form a belief about their relative likelihood. In fact, Bernstein axiomatically pursued just such a concept even before Kolmogorov (Bernstein, 1917)! ${ }^{10}$

This article's question is one of harmony: when do the concepts of cardinal probabilities and ordinal beliefs coincide? When is it as though instead of comparing two propositions with a balance, one instead compares them using a scale; first weighing the first proposition, then weighing the second, and finally comparing the two weights? What assumptions, exactly, are we making when we model someone's beliefs with a probability measure? We categorize the previous literature dedicated to this question by which of four assertions is imposed, emphasizing that our contribution is to proceed without any of these assertions.

\section{The literature with no atoms}

The seminal contributions to the qualitative probability literature (Bernstein, 1917; de Finetti, 1937; Koopman, 1940) imposed that $S$ can be partitioned into an arbitrarily large number of equally likely events. This implies that there is a unique measure that "almost represents" $\succsim$, though it may assign the same probability to two distinguished events (see Kreps, 1988).

To guarantee representation, Savage (1954) imposed a stronger axiom, fineness-andtightness, which moreover implies that the representation's range is $[0,1] .{ }^{11}$ Surprisingly,

while Stone's result relies on the Boolean Prime Ideal Theorem, which is weaker than the Axiom of Choice (Halpern and Lévy, 1964), the result of Loomis and Sikorski does not (Buskes, de Pagter, and van Rooij, 2008), nor does the fact that no generality is lost for finite algebras (Birkhoff, 1937).

${ }^{9}$ As Savage (1961) explains: "Whatever else a frequentist may do with the results of an investigation he cannot, as a frequentist, use them to calculate probabilities of the uncertain propositions that are under investigation. Technically, this means that he is cut off from most applications of Bayes' theorem, the algorithm for calculating what the new probability of a proposition is on the basis of its original probability and new relevant evidence. One's natural inclination to ask, 'To what degree of conviction does this new data entitle me?' usually must be, and has been, regarded as a nonsense question by the frequentist. The frequentist is required, therefore, to seek a concept of evidence, and of reaction to evidence, different from that of the primitive, or natural, concept that is tantamount to application of Bayes' theorem."

${ }^{10}$ At the time, Bernstein's work was not well-known outside of Russia, and today the independent works of de Finetti and Koopman remain better-known (de Finetti, 1937; Koopman, 1940). Slutsky (1922) is another important Russian contribution toward axiomatic probability from this time; citing it in his obituary of Slutsky, Kolmogorov (1948) remarked that Slutsky "was the first to draw a correct picture of the purely mathematical essence of probability theory." Slutsky (1925), in turn, remarked: "Regrettably, in 1922 I was not acquainted with Bernstein (1917) where the indicated ideas had already been realized, and more formally at that. That contribution deserves to be most seriously studied." See Sheynin (2010) for a discussion, and see Sheynin (2005) for a translation of Bernstein (1917).

${ }^{11}$ For simplicity of presentation, Savage further imposed that $\mathcal{A}=2^{S}$, but his analysis is valid if $\mathcal{A}$ is any $\sigma$-algebra (Wakker, 1981; Wakker, 1993a). If $\mathcal{A}$ is only required to be an algebra and a weaker 
Savage's axioms are in fact compatible when $|S|=|\mathbb{N}|$; they are only incompatible when $S$ is finite (Kreps, 1988; Wakker, 1993a). That said, Savage's axioms are incompatible with atoms, and they are incompatible with $\sigma$-additivity when $|S|=|\mathbb{N}|{ }^{12}$

Savage deliberately avoided any continuity axiom implying $\sigma$-additivity, explaining: "I know of no argument leading to the requirement of countable additivity[... ] it therefore seems better not to assume countable additivity outright as a postulate, but to recognize it as a special hypothesis yielding, where applicable, a large class of useful theorems." But there have since been two particularly strong such arguments: first, $\sigma$-additivity is required for avoiding money pumps (Adams, 1962; Seidenfeld and Schervish, 1983), and second, $\sigma$-additivity is required to ensure that choice always respects strict first order stochastic dominance (Wakker, 1993b). Based on these observations and others, Stinchcombe (1997) argues that a measure which is only finitely-additive indicates a misspecified state space: "One summary[... ] is that countably infinite constructions require countably additive probabilities."

Villegas (1964) identified the appropriate continuity axiom: if $\mu$ is a measure representation of $\succsim$, then $\mu$ is a $\sigma$-additive if and only if $\succsim$ is monotonely continuous (Theorem V1). Furthermore, (i) monotone continuity, and (ii) the requirement that there are no atoms, are sufficient for $\sigma$-measure representation (Theorem V2). We appeal to these important results directly in our proof. Unfortunately, these axioms are incompatible when $|S| \leq|\mathbb{N}|$.

While Kopylov (2010) does not explicitly study qualitative probabilities, it is clear from his analysis that $\sigma$-measure representation is guaranteed by imposing only strong monotone continuity when $\mathcal{A}$ is countably separated (Mackey, 1957). That there are no atoms is then implied. Because Kopylov's axioms are incompatible when $|S| \leq|\mathbb{N}|$, and because $\mathcal{A}$ can only be countably separated when $|S| \leq|\mathbb{R}|,{ }^{13}$ it follows that under the Continuum Hypothesis, this approach is custom-tailored to the case where $|S|=|\mathbb{R}|$.

Our results generalize that of Villegas (1964) by weakening the requirement that there are no atoms. Like Savage (1954), our axioms are incompatible only when $S$ is finite. Unlike the rest of this part of the literature, our results are compatible with atoms, and with $\sigma$-additivity whenever $S$ is infinite.

\section{The literature with cancellation}

Even when $S$ is finite, there are qualitative probabilities without measure representations (Kraft, Pratt, and Seidenberg, 1959). This was first demonstrated by means of

version of fineness-and-tightness is imposed, then a measure representation is still guaranteed (Wakker, 1981), but its range need only be a dense subset of [0,1] (Marinacci, 1993). While there are indeed such atomless measures without convex range (Nunke and Savage, 1952), every atomless $\sigma$-measure has convex range (see Halmos, 1947, Lemma 2).

${ }^{12}$ Savage's simplifying assumption that $\mathcal{A}=2^{S}$ seriously restricts compatibility with $\sigma$-additivity. If $|S|=|\mathbb{R}|$, then the existence of an atomless $\sigma$-measure defined on $2^{S}$ is inconsistent with the Continuum Hypothesis (Banach and Kuratowski, 1929; Ulam, 1930). Savage avoids this issue by relaxing $\sigma$-additivity, as there are well-behaved atomless measures - for example when $S=[0,1]$, there are measures that (1) agree with the Lebesgue measure on those sets where it is defined, and (2) assign the same number to any pair of congruent sets (Banach, 1932). By contrast, we avoid this issue both by allowing $\mathcal{A}$ to be any $\sigma$-algebra and by allowing atoms.

${ }^{13} \mathcal{A}$ is countable separated if there is a countable collection of events $\mathcal{A}^{*} \subseteq \mathcal{A}$ such that for each distinct pair $s, s^{\prime} \in S$, there is $A \in \mathcal{A}^{*}$ with $s \in A$ and $s^{\prime} \notin A$. That this implies $|S| \leq|\mathbb{R}|$ can be seen from the following argument of Faris (2007): index $\mathcal{A}^{*}$ by the natural numbers, so that $\mathcal{A}^{*}=\left\{A_{1}, A_{2}, \ldots\right\}$, and define the mapping $\varphi$ from $S$ to the Cantor set $\mathbb{C}$ (Cantor, 1883) by defining, for each $i \in \mathbb{N}$ and each $s \in S, \varphi_{i}(s) \equiv \mathbb{1}_{A_{i}}(s)$. Then $\varphi$ is an injection, so $|S| \leq|\mathbb{C}|=|\mathbb{R}|$. 
a qualitative probability $\succsim$ on the subsets of $\left\{s_{1}, s_{2}, s_{3}, s_{4}, s_{5}\right\}$ that includes the comparisons:

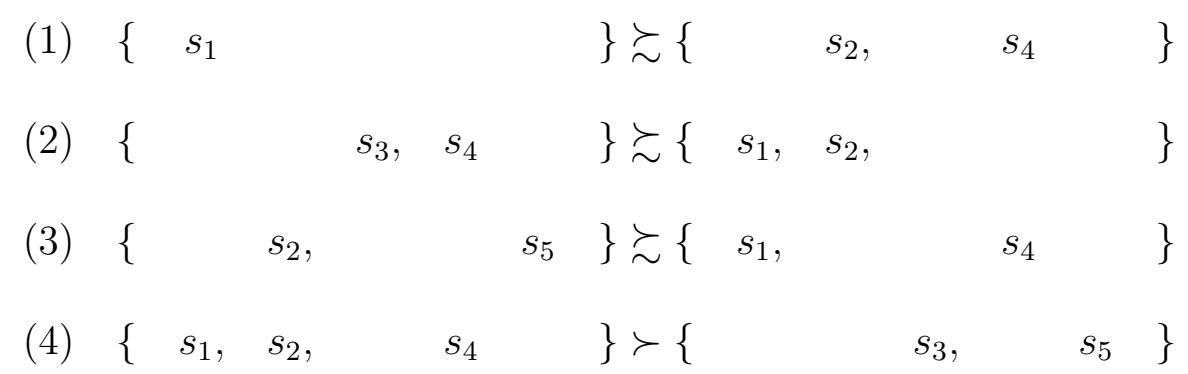

A necessary and sufficient condition for measure representation is finite cancellation (Kraft, Pratt, and Seidenberg, 1959; Scott, 1964; Fishburn, 1970; Krantz, Luce, Suppes, and Tversky, 1971), the assertion that there is no such list of comparisons. This condition is quite strong, alone necessary and sufficient for additive representation (Fishburn, 1970). Attempts to justify this axiom generally rely on enriched models - for example, preferences over multi-sets of states, where such a multi-set is interpreted as a portfolio of Arrow-Debreu-McKenzie securities - and as such it can be difficult to interpret.

Stronger and more complex conditions have been identified that guarantee measure representation while allowing atoms when $S$ is infinite (Domotor, 1969; Chateauneuf and Jaffray, 1984; Chateauneuf, 1985). Chateauneuf (1985) in fact provides necessary and sufficient conditions using an axiom that implies finite cancellation. We recommend Fishburn's survey for more information (Fishburn, 1986).

By contrast, we do not impose any cancellation axiom. For the purely discrete case, we instead follow Cantor and Debreu (Cantor, 1895; Debreu, 1954; Debreu, 1964) by constructing an order-dense family of equivalence classes. We then define a binary operation on this family and, following Peano (1889), show that this binary operation interacts appropriately with succession - that is, addition by one - from which we derive cancellation, commutativity, associativity, and all other properties of ordinary addition.

\section{The literature with solvability}

To our knowledge, there are two articles studying qualitative probabilities with atoms that do not impose cancellation (Abdellaoui and Wakker, 2005; Chew and Sagi, 2006). Both approaches involve solvability: ${ }^{14}$ for each pair of disjoint events, there is a subevent of one that is as likely as the other. Abdellaoui and Wakker (2005) allow for mosaics instead of $\sigma$-algebras and allow for measures that are not convex-ranged, while Chew and Sagi (2006) work with an ordering of events induced from preferences over acts through "exchangeability" and proceed without monotonicity. In both cases there may be atoms, provided any pairwise disjoint collection of atoms is finite with equally-likely members.

By contrast, our results are compatible with an 'exhaustive' pairwise disjoint collection of atoms which is finite or infinite, and moreover, the atoms need not be equally-likely. For the discrete case, we explain the difference in approach with a visual metaphor where size corresponds to likelihood. Given two events, one larger than the other, we would often like to find a piece of the larger that is the same size as the smaller. In the solvability approach, the larger event is made of a fabric from which the desired piece can be cleanly cut. In our approach, we cannot always create the desired piece because the larger event

\footnotetext{
${ }^{14}$ This is not quite the language used in either article. Abdellaoui and Wakker (2005) use "solvability" to refer to a stronger axiom, while Chew and Sagi (2006) use "completeness" to refer to the given axiom and use "solvability" to refer to a related property for measures.
} 
may consist of blocks that cannot be cut. Nevertheless, whenever the larger event has a rich enough composition, the desired piece can be mosaicked by iteratively adding smaller and smaller blocks to better-approximate the specified size.

\section{The literature with additional primitives}

We refer to works that use the entire Savage (1954) model: a state space $S$, an outcome space $X$, and preferences over acts $f: S \rightarrow X$. Often implicitly, $\mathcal{A}=2^{S}$. To uncover the embedded qualitative probability, first select a pair of outcomes $x^{*}$ and $x$ such that the act guaranteeing $x^{*}$ is preferred to that guaranteeing $x$. Beliefs can then be defined as preferences among simple bets: $A \succsim B$ if and only if the act returning $x^{*}$ on $A$ and $x$ otherwise is at least as desirable as the act returning $x^{*}$ on $B$ and $x$ otherwise. It is imposed that this is well-defined; $\succsim$ does depend on the choice of $x^{*}$ and $x$.

Savage used only $\succsim$ to deliver the unique measure representation of beliefs. But to handle atoms that are not equally likely without using a cancellation or solvability axiom, others have used the entire model, particularly when $S$ is discrete. Typically this involves imposing, at a minimum, that $X$ has cardinality of at least the continuum and that $X$ has a rich topological structure. For example, $X$ might be a simplex of objective "roulette lotteries" that can be mixed (as in Anscombe and Aumann, 1963 and the vast literature that followed), or an interval of dollar amounts (see Wakker, 1989; Gul, 1992; and references therein), or Euclidean commodity space (as in the literature on intertemporal preferences, for example Koopmans, 1960).

In contrast to those who use the full Savage model under the uncertainty interpretation, we do not rely on any extraneous assumptions about some space of outcomes to deliver the measure representation, keeping our analysis firmly focused on beliefs. In contrast to most studies of intertemporal preference, our approach allows for finite consumption spaces; we revisit this point in the Conclusion.

\subsection{Outline}

In Section 2, we provide the model and formally state our five theorems. We first define (ordinal) qualitative probability, (cardinal) quantitative probability, and representation of the former by the latter, then formally state our main result (Theorem 5). We then introduce catalogued spaces where each atom has a singleton core, and formally state the other four theorems.

The next four sections are dedicated to the techniques involved in the proof of Theorem 5. (Structurally, Theorem 3 is one of many lemmas in this proof.) In particular:

- Section 3 is dedicated to the proof of Theorem 1, which states that monotone continuity and 3-AS guarantee $\sigma$-measure representation for the discrete catalogued spaces, where $|S|=|\mathbb{N}|$ and each singleton is a core. The overall strategy is to construct a particular kind of order-dense family of equivalence classes called a supercabinet; some of the key techniques involve viewing the events as members of the Cantor set, constructing specified events using a greedy algorithm, and verifying addition axioms similar to Peano's for a particular binary operation.

- Section 4 is dedicated to the proof of Theorem 2, which states that monotone continuity and 3-AS guarantee $\sigma$-measure representation for catalogued spaces with a finite number of cores. The proof involves partitioning an infinitely-divisible event into 'honorary cores' and applying the techniques from the previous section. 
- Section 5 is dedicated to the proof of Theorem 4, which states that monotone continuity and $3^{+}-A S$ guarantee $\sigma$-measure representation for the remaining catalogued spaces, where there is a messy sequence of cores together with some infinitelydivisible events. One of the key lemmas is Theorem 3, which states that monotone continuity and unlikely cores guarantee $\sigma$-measure representation. Constructing the $\sigma$-measure involves repeatedly splitting the space into a left space and a right space, then applying Theorem 2 to the former and Theorem 3 to the latter. Verifying that the $\sigma$-measure is in fact a representation involves an algorithm that takes as input an arbitrary pair of events $A$ and $B$, and returns as output a finite sequence of event pairs, allowing the statement " $A \succsim B$ if and only if $\mu(A) \geq \mu(B)$ " to be directly proved.

- Section 6 briefly describes how Theorem 5 is established by the above results.

Finally, Section 7 concludes by discussing two alternative settings where our results are relevant: preferences over parts of a heterogeneous good and preferences over consumption streams.

\section{Model and main results}

\subsection{Qualitative probability}

A likelihood space $(S, \mathcal{A}, \succsim)$ is a triple consisting of

(1) a set of states $S$ with generic members $s, s^{\prime}$,

(2) a $\sigma$-algebra of events $\mathcal{A} \subseteq 2^{S}$, including $S$, with generic members $A, B, C,{ }^{15}$ and

(3) a likelihood relation $\succsim$, which is a binary relation on $\mathcal{A}$.

We interpret $\succsim$ as a collection of comparisons of events on the basis of relative likelihood: $A \succsim B$ denotes " $A$ is at least as likely as $B$." We write $A \sim B$ to denote $A \succsim B$ and $B \succsim A$, and write $A \succ B$ to denote $A \succsim B$ but not $A \sim B$. We emphasize that, for convenience, we include degenerate triples $(\emptyset,\{\emptyset\}, \succsim)$ as likelihood spaces.

Formally, all our results concern likelihood spaces. That said, we often implicitly take $S$ and $\mathcal{A}$ as the set of states and $\sigma$-algebra, respectively, abusing notation by referring to the likelihood space by its likelihood relation. In certain cases, we are instead explicit about the entire likelihood space, particularly when comparing several likelihood spaces or when imposing structure on the set of states and $\sigma$-algebra.

The following assumptions are standard:

○ ORDER: ${ }^{16}$ The relation $\succsim$ is complete and transitive.

${ }^{15}$ Savage (1954) is less general, asserting that $\mathcal{A}=2^{S}$, while others are more general, allowing $\mathcal{A}$ to be any "abstract" $\sigma$-algebra (Villegas, 1964; Villegas, 1967), or any algebra (Wakker, 1981; Marinacci, 1993), or any mosaic (Kopylov, 2007; Abdellaoui and Wakker, 2005). This last generalization is motivated by the distinction between "risk" and "ambiguity" (Knight, 1921) illustrated by the Ellsberg paradox (Ellsberg, 1961), as the collection of "subjectively risky events" (Epstein and Zhang, 2001) need only be a mosaic.

${ }^{16}$ The standard assumption of completeness - that comparisons are always possible - has been criticized on both normative and positive grounds (von Neumann and Morgenstern, 1947; Aumann, 1962; Schmeidler, 1989; Mandler, 2005). Indecisiveness can be observed and distinguished from indifference (Eliaz and Ok, 2006), and in fact has been in a recent experiment (Cettolin and Riedl, 2013). Recently, likelihood relations have been considered without completeness, and in particular, axiomatic foundations 
- Separability: ${ }^{17}$ For each triple $A, B, C \in \mathcal{A}$ such that $A \cap C=B \cap C=\emptyset$,

$$
A \succsim B \text { if and only if } A \cup C \succsim B \cup C \text {. }
$$

○ Monotonicity: For each pair $A, B \in \mathcal{A}, A \subseteq B$ implies $B \succsim A$.

- Nondegeneracy: There are $A, B \in \mathcal{A}$ such that $A \succ B$.

We gather these standard assumptions in the following definition:

Definition: A qualitative probability space is a likelihood space that satisfies order, separability, monotonicity, and nondegeneracy. A qualitative probability is the likelihood relation of a qualitative probability space. ${ }^{18}$

\subsection{Quantitative probability}

We seek conditions under which our ordinal notion of qualitative probability space coincides with the standard cardinal notion of (quantitative) probability space (Kolmogoroff, 1933). The cardinal notion is that of a probability measure:

Definition: A function $\mu: \mathcal{A} \rightarrow[0,1]$ is a measure if

(1) $\mu(S)=1$, and

(2) for each finite, pairwise disjoint collection $\left\{A_{i}\right\}_{i \in I} \subseteq \mathcal{A}$,

$$
\mu\left(\cup A_{i}\right)=\sum \mu\left(A_{i}\right) .
$$

A measure $\mu$ is moreover a $\sigma$-measure if

(3) for each countably infinite, pairwise disjoint collection $\left\{A_{i}\right\}_{i \in I} \subseteq \mathcal{A}$,

$$
\mu\left(\cup A_{i}\right)=\sum \mu\left(A_{i}\right) .
$$

Let $\mathbb{M}(\mathcal{A}) \subseteq[0,1]^{\mathcal{A}}$ denote the set of measures and let $\mathbb{M}^{\sigma}(\mathcal{A}) \subseteq \mathbb{M}(\mathcal{A})$ denote the set of $\sigma$-measures.

We say that the ordinal and cardinal notions coincide when the measure represents likelihood comparisons:

Definition: If $(S, \mathcal{A}, \succsim)$ is a qualitative probability space and $\mu \in \mathbb{M}(\mathcal{A})$, then we say $\mu$ is a representation of $(S, \mathcal{A}, \succsim)$ if for each pair $A, B \in \mathcal{A}$,

$$
A \succsim B \text { if and only if } \mu(A) \geq \mu(B) \text {. }
$$

In this case we say $\mu$ represents $\succsim$.

for representation by multiple prior probability measures - where a first event is more likely than a second if and only if each prior assigns a higher probability to the first - have been provided, both when each event can be partitioned into two equally-likely subevents (Nehring, 2009) and more generally through the use of a cancellation axiom (Alon and Lehrer, 2014).

${ }^{17}$ We note that when likelihood relations are interpreted as observable preferences among bets, the systematic violation of this axiom has been observed in an experiment where certain events are attached to probability appraisals while others are not (Ellsberg, 1961); the favoring of the appraised events is a phenomenon typically ascribed to ambiguity aversion (though not always; see Ergin and Gul, 2009).

${ }^{18}$ Savage (1954) and others give an equivalent definition using a weaker version of monotonicity and a stronger version of nondegeneracy. 


\subsection{The main result}

As argued in Section 1.1, there are strong reasons to impose that the measure representation is $\sigma$-additive (Adams, 1962; Seidenfeld and Schervish, 1983; Wakker, 1993b; Stinchcombe, 1997), and the appropriate axiom for doing so has been identified: ${ }^{19}$

○ Monotone continuity (Villegas, 1964): For each $A \in \mathcal{A}$ and each $\left(B_{i}\right) \in \mathcal{A}^{\mathbb{N}}$, if

(i) $B_{1} \supseteq B_{2} \supseteq \ldots$, and

(ii) for each $i \in \mathbb{N}, B_{i} \succsim A$,

then $\cap B_{i} \succsim A$.

Theorem V1 (Villegas, 1964): If $(S, \mathcal{A}, \succsim)$ is a qualitative probability space with representation $\mu \in \mathbb{M}(\mathcal{A})$, then $(S, \mathcal{A}, \succsim)$ satisfies monotone continuity if and only if $\mu \in \mathbb{M}^{\sigma}(\mathcal{A})$.

We therefore impose monotone continuity, whose implications are understood when there are no 'atoms,' or events whose less-likely subevents are null:

Definition: An atom is an event $A \in \mathcal{A}$ such that

(1) $A \succ \emptyset$, and

(2) for each $B \subseteq A$, either $B \sim A$ or $B \sim \emptyset$.

We write $\mathcal{A}^{\alpha} \subseteq \mathcal{A}$ for the collection of atoms.

In particular, when there are no atoms, monotone continuity guarantees a unique representation by a special kind of measure:

Definition: A measure $\mu \in \mathbb{M}(\mathcal{A})$ is infinitely-divisible if for each $A \in \mathcal{A}$ and each $\lambda \in[0,1]$, there is $B \subseteq A$ such that $\mu(B)=\lambda \mu(A)$.

Theorem V2 (VIllegas, 1964): If $(S, \mathcal{A}, \succsim)$ is a qualitative probability space satisfying monotone continuity such that $\mathcal{A}^{\alpha}=\emptyset$, then it has a unique representation $\mu \in \mathbb{M}^{\sigma}(\mathcal{A})$. Moreoever, $\mu$ is infinitely-divisible.

In this article, we generalize Theorem V2 by allowing atoms. To do so, we introduce a parametric family of atom-swarming axioms, with higher-parameter assumptions logically stronger than lower-parameter ones. Formally, for each $A \in \mathcal{A}$, let $(\downarrow A)$ denote the collection of non-null events that are less likely than $A$ :

$$
(\downarrow A) \equiv\left\{A^{\prime} \in \mathcal{A} \mid A \succ A^{\prime} \succ \emptyset\right\} .
$$

For each $k \in \mathbb{N}$, we define the axiom that for each atom $A, \cup(\downarrow A)$ is, in an ordinal sense, $k$ times as likely as $A$ :

○ $k$ TH-ORDER ATOM-SWARMing $\left(k\right.$-AS): For each $A \in \mathcal{A}^{\alpha}$, there are

(i) pairwise-disjoint $\mathcal{B} \subseteq(\downarrow A)$, and

${ }^{19}$ In fact, this is the appropriate axiom even in the multiple priors model (Gilboa and Schmeidler, 1989 ), guaranteeing that the set of priors is a relatively weak compact set of $\sigma$-measures (Chateauneuf, Maccheroni, Marinacci, and Tallon, 2005). 
(ii) $\mathcal{B}_{1}, \mathcal{B}_{2}, \ldots, \mathcal{B}_{k} \subseteq \mathcal{B}$ partitioning $\mathcal{B}$,

such that for each $i \in\{1,2, \ldots, k\},\left(\cup \mathcal{B}_{i}\right) \succsim A$.

If furthermore, for each $i \in\{1,2, \ldots, k\},\left(\cup \mathcal{B}_{i}\right) \succ A$, then strict $k$ th-order atomswarming, or $k^{+}-A S$, is satisfied.

As promised, higher-indexed axioms are logically stronger. Note that under monotone continuity, any pairwise-disjoint collection of non-null events $\mathcal{B}$ is countable, so if $\mathcal{B}_{i}$ is a subcollection of some such $\mathcal{B}$, then $\left(\cup \mathcal{B}_{i}\right)$ is an event.

Our main result is that $\sigma$-representation is guaranteed by monotone continuity and $3^{+}-A S$ :

TheOrEm 5: A triple $(S, \mathcal{A}, \succsim)$ is a qualitative probability space satisfying monotone continuity and $3^{+}-A S$ if and only if it has a unique representation $\mu \in \mathbb{M}^{\sigma}(\mathcal{A})$ such that for each $A \in \mathcal{A}$,

(i) if $A$ is an atom, $\mu(\cup(\downarrow A))>3 \mu(A)>0$, and

(ii) if $A$ contains no atoms, for each $\lambda \in[0,1]$, there is $B \subseteq A$ such that $\mu(B)=\lambda \mu(A)$.

\subsection{Catalogued spaces and other results}

Working with atoms involves some nuance: two distinct atoms can have non-empty intersection; in fact, one atom can even be contained in another. This makes it tempting to consider only 'smallest' atoms: for each atom, take the collection of its non-null subevents (which are themselves atoms), then take the intersection of this collection. The problem is that in general, this collection need not be countable, and therefore this intersection need not be an event.

We suppress the above nuance for most of the analysis by asserting that each atom contains a core, or a singleton atom, and do so by working with the following class of qualitative probability spaces:

Definition: A qualitative probability space $(S, \mathcal{A}, \succsim)$ is catalogued if there is $S^{\oplus} \subseteq S$ such that

(1) either

(i) $S^{\oplus}=\emptyset$,

(ii) there is $m \in \mathbb{N}$ such that $S^{\oplus}=\{1,2, \ldots, m\}$, or

(iii) $S^{\oplus}=\mathbb{N}$,

(2) for each $s \in S^{\oplus},\{s\} \in \mathcal{A}^{\alpha}$,

(3) for each $A \subseteq S \backslash S^{\oplus}, A \notin \mathcal{A}^{\alpha}$, and

(4) for each pair $s, s^{\prime} \in S^{\oplus}, s<s^{\prime}$ implies $\{s\} \succsim\left\{s^{\prime}\right\}$.

We let $\mathcal{A}^{\oplus}$ denote $2^{S^{\oplus}}$, we let $S^{\circ}$ denote $S \backslash S^{\oplus}$, and we let $\mathcal{A}^{\circ}$ denote $\mathcal{A} \cap 2^{S^{\circ}}$. We say that $(S, \mathcal{A}, \succsim)$ is

- purely- $\sigma$-catalogued if $S^{\oplus}=S=\mathbb{N}$,

- finitely-catalogued if $S^{\oplus} \neq \mathbb{N}$, and

- $\sigma$-catalogued if $S^{\oplus}=\mathbb{N}$. 


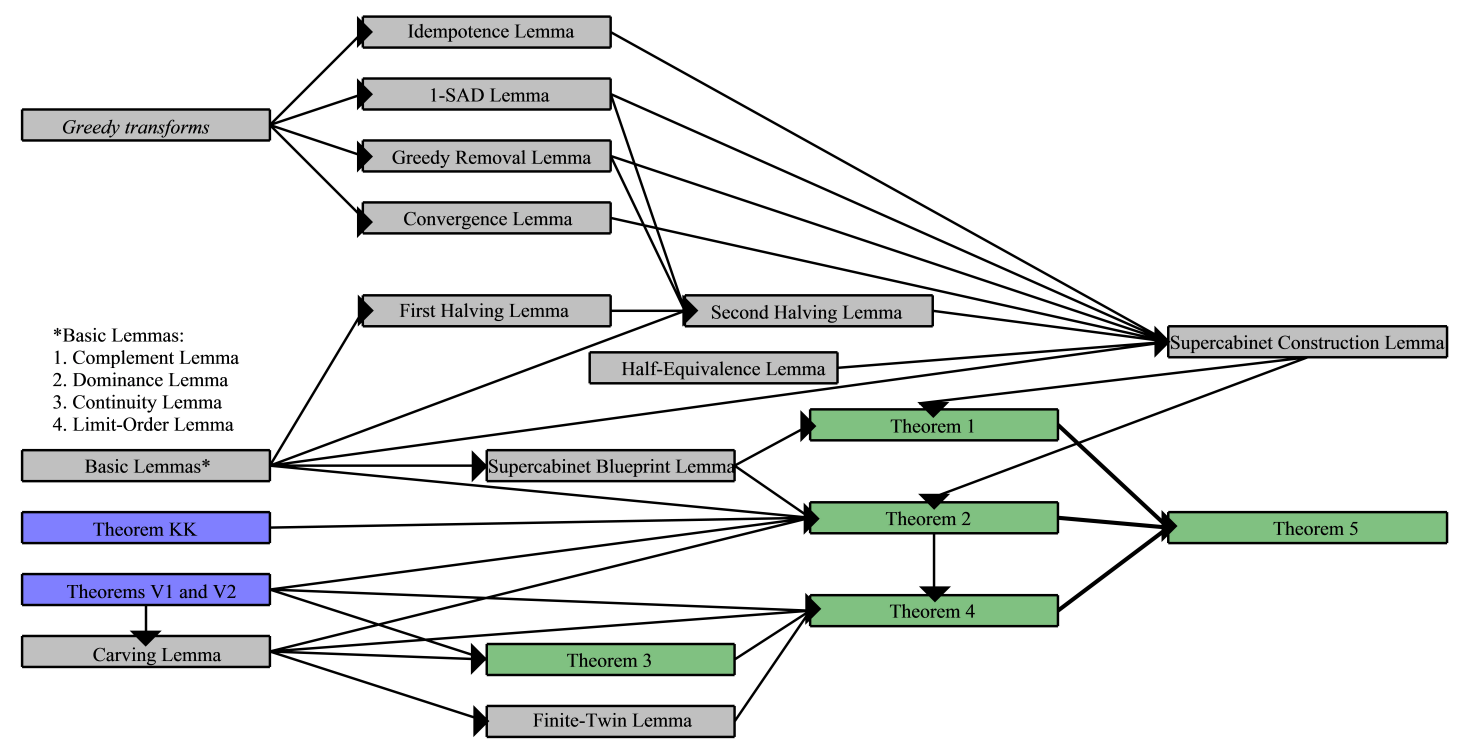

Figure 2: The results and their relationships. There is an arrow from a first result to a second if the first result is used at some point in the proof of the second. Blue results are due to others, green results are theorems, and the main result is Theorem 5 .

Thus in a catalogued space, each atom has a core $\{s\}$, where $s \in S^{\oplus}$. Moreover, these singletons are labeled by consecutive natural numbers so that $\{1\} \succsim\{2\} \succsim \ldots$, which will frequently be convenient.

We provide another generalization of Theorem V2 for catalogued spaces, using the requirements that the cores are not more likely than their complement:

○ UNLIKELY CORES: A catalogued probability space satisfies the axiom if $S^{\circ} \succsim S^{\oplus}$.

THEOREM 3: If $(S, \mathcal{A}, \succsim)$ is a catalogued qualitative probability space satisfying monotone continuity and unlikely cores, then it has a unique representation $\mu \in \mathbb{M}^{\sigma}(\mathcal{A})$.

Structurally, the other three theorems are the supporting pillars of Theorem 5, and the next three sections are dedicated to explaining their proofs. Theorem 1, Theorem 2, and Theorem 4 each provide sufficient conditions for $\sigma$-measure representation of certain catalogued spaces - in particular, for purely- $\sigma$-catalogued spaces, finitely-catalogued spaces, and $\sigma$-catalogued spaces, respectively:

THEOREM 1: If $(S, \mathcal{A}, \succsim)$ is a purely- $\sigma$-catalogued qualitative probability space satisfying monotone continuity and 3-AS, then it has a unique representation $\mu \in \mathbb{M}^{\sigma}(\mathcal{A})$.

Theorem 2: If $(S, \mathcal{A}, \succsim)$ is a finitely-catalogued qualitative probability space satisfying monotone continuity and $3-A S$, then it has a unique representation $\mu \in \mathbb{M}^{\sigma}(\mathcal{A})$.

THEOREM 4: If $(S, \mathcal{A}, \succsim)$ is a $\sigma$-catalogued qualitative probability space satisfying monotone continuity and $3^{+}-A S$, then it has a unique representation $\mu \in \mathbb{M}^{\sigma}(\mathcal{A})$.

The theorems are numbered according to the sequence they are proved in the following sections. For a schematic of the overall proof structure, see Figure 2. 


\section{Proof sketch for Theorem 1}

\subsection{Supercabinet blueprint}

We begin by investigating purely $\sigma$-catalogued probability spaces: $S$ is the natural numbers; $\mathcal{A}$ is its power set; and the singletons are the cores, which are labeled in nonincreasing order of likelihood. For both cardinal and ordinal purposes, it is fruitful to consider $\mathcal{A}$ with the topology of pointwise convergence: for measures, $\sigma$-additivity is equivalent to continuity (with this topology), while for qualitative probabilities, monotone continuity is equivalent to closure of upper and lower contour sets (with this topology). ${ }^{20}$

We can thus restate our objective: we seek to prove that there is a continuous representation of $\succsim$ that is moreover a measure. A first step might be to establish that there is some continuous representation, and toward this end it is helpful to recall a classic result from the utility function literature, Theorem CD (Cantor, 1895; Debreu, 1954; Debreu, 1964). This theorem involves a structured family of equivalence classes, which I will suggestively refer to as a cabinet. ${ }^{21}$

Definition: For each qualitative probability space $(S, \mathcal{A}, \succsim)$, a collection of equivalence classes $\left\{\mathcal{Z}_{i}\right\}_{i \in \mathbb{N}} \subseteq[\mathcal{A} / \sim]$ is a cabinet of $(S, \mathcal{A}, \succsim)$ if for each pair $A, B \in \mathcal{A}$ such that $A \succ B$, there is $Z \in \cup \mathcal{Z}_{i}$ such that $A \succsim Z \succsim B$.

A cabinet can be thought of as a collection of drawers labeled $1,2,3, \ldots$, each drawer filed with equally-likely events. The requirement is that if I give a file clerk two events $A$ and $B$, one more likely than the other, then he should be able to go to the cabinet, open one of the drawers, and retrieve some event $Z$ that is between $A$ and $B$ in likelihood. Theorem CD states that if we can construct such a cabinet, then we are promised a continuous representation:

Theorem CD (Cantor, 1895; Debreu, 1954; Debreu, 1964): ${ }^{22}$ If (i) $(S, \mathcal{A}, \succsim)$ has a cabinet, and (ii) $\succsim$ has closed upper and lower contour sets, then there is continuous $u: \mathcal{A} \rightarrow \mathbb{R}$ such that

$$
u(A) \geq u(B) \Leftrightarrow A \succsim B .
$$

For our objective, we do not want just any continuous representation; we want one that is moreover a measure, taking into account the algebraic structure of the events. We therefore do not want just any cabinet; we want one that moreover takes into account the algebraic structure of the events. What I propose is that we first repaint the cabinet, labeling the drawers not with $1,2,3, \ldots$, but rather in anticipation of probabilities: let $v$

\footnotetext{
${ }^{20}$ I was unable to find a reference for either of these observations, though I would not be surprised if they are already known, especially the former.

${ }^{21}$ This pedagogical device was inspired by a recorded lecture of physicist Richard Feynman, where he explains how a computer can be viewed as an extremely fast but extremely stupid file clerk.

${ }^{22}$ Cantor first proved the result under the stronger $A \succ Z \succ B$ assumption (Cantor, 1895). Nearly six decades later - at the suggestion of Savage, according to the acknowledgment-Debreu attempted to prove the result under the weaker $A \succsim Z \succsim B$ assumption (Debreu, 1954). Debreu then noticed an error in his own proof, which he corrected a decade later using his acclaimed Gap Lemma (Debreu, 1964). For narrative clarity, we have chosen a weaker statement than that in Debreu (1964). In fact $\mathcal{A}$ need not be a $\sigma$-algebra, and without assumption (ii), Debreu proves there is a representation $u$ which is upper semi-continuous in any topology for which upper contour sets are closed and lower semi-continuous in any topology for which lower contour sets are closed.
} 
label the drawer filed with events to which we should like to eventually assign probability $v$. Since we can only choose a countably infinite collection of anticipated probabilities from $[0,1]$ as labels, I propose we take the dyadic rationals, $\mathcal{2} \subseteq[0,1]$ :

$$
\mathcal{L} \equiv\left\{\sum_{i \in F}\left(\frac{1}{2}\right)^{i}|F \subseteq \mathbb{N},| F|<| \mathbb{N} \mid\right\} \cup\{1\} .
$$

The dyadic rationals can be thought of as the labels appearing anywhere in a sequence of progressively-finer rulers, the first ruler measuring halves of a foot, the second ruler measuring fourths, the third ruler measuring eights, and in general the $k$ th ruler measuring $\left(\frac{1}{2^{k}}\right)$ ths.

Of course, it is not enough to simply repaint the cabinet; these new labels must be operationalized:

Definition: For each qualitative probability space $(S, \mathcal{A}, \succsim)$, a collection of equivalence classes $\left\{\mathcal{Z}_{v}\right\}_{v \in \mathbb{2}} \subseteq[\mathcal{A} / \sim]$ is a supercabinet of $(S, \mathcal{A}, \succsim)$ if

[SC1] $\emptyset \in \mathcal{Z}_{0}$ and $S \in \mathcal{Z}_{1}$,

[SC2] for each pair $v, v^{\prime} \in \mathcal{Z}$ such that $v+v^{\prime} \leq 1$, there are disjoint $Z_{v} \in \mathcal{Z}_{v}$ and $Z_{v^{\prime}} \in \mathcal{Z}_{v^{\prime}}$ such that $Z_{v} \cup Z_{v^{\prime}} \in \mathcal{Z}_{v+v^{\prime}}$

[SC3] for each non-increasing $\left(v_{i}^{+}\right) \in \mathbb{2}^{\mathbb{N}}$ and non-decreasing $\left(v_{i}^{-}\right) \in 2^{\mathbb{N}}$ such that $\lim v_{i}^{+}=\lim v_{i}^{-},{ }^{23}$ there are are convergent $\left(A_{i}^{+}\right),\left(A_{i}^{-}\right) \in \mathcal{A}^{\mathbb{N}}$ such that

(i) for each $i \in \mathbb{N}, A_{i}^{+} \in \mathcal{Z}_{v_{i}^{+}}$and $A_{i}^{-} \in \mathcal{Z}_{v_{i}^{-}}$, and

(ii) $\lim A_{i}^{+}=\lim A_{i}^{-}$.

[SC4] for each monotonic pair $\left(v_{i}\right),\left(w_{i}\right) \in \mathcal{2}^{\mathbb{N}}$ such that for each $i \in \mathbb{N}, v_{i}+w_{i} \leq 1$, there are convergent $\left(A_{i}\right),\left(B_{i}\right) \in \mathcal{A}^{\mathbb{N}}$ such that for each $i \in \mathbb{N}, A_{i}$ and $B_{i}$ are disjoint with $A_{i} \in \mathcal{Z}_{v_{i}}$ and $B_{i} \in \mathcal{Z}_{w_{i}}$.

Though it is not obvious by comparing the definitions, a supercabinet is a special kind of cabinet; this follows from our next lemma. The key idea behind the additional structure is given by [SC2]: if I give a file clerk two anticipated probabilities $v$ and $v^{\prime}$, and their sum does not exceed 1 , then he should be able to take an event $Z_{v}$ from the drawer labeled $v$ and an event $Z_{v^{\prime}}$ from the drawer labeled $v^{\prime}$. Moreover, he should be able to choose this pair so that $Z_{v}$ and $Z_{v^{\prime}}$ are disjoint. Finally, he should be able to find their union $Z_{v} \cup Z_{v^{\prime}}$ filed in the drawer labeled $v+v^{\prime}$. The following lemma promises that if we can construct a supercabinet, then we are done:

Supercabinet Blueprint Lemma: If $(S, \mathcal{A}, \succsim)$ is a qualitative probability space that has a supercabinet and satisfies monotone continuity, then there is a unique $\mu \in \mathbb{M}^{\sigma}(\mathcal{A})$ such that $\mu$ represents $\succsim$.

As expected, for each $v \in \mathcal{L}$, the unique measure representation assigns to each event in $\mathcal{Z}_{v}$ the probability $v$; indeed by design, any measure representation must. A continuity argument takes care of the un-filed events; see Appendix 2 for the proof.

With the blueprint in hand, let us begin construction.

${ }^{23}$ Since both sequences are bounded, they are also convergent. 


\subsection{Constructing halves}

It is easy enough to file the empty set (and those events equally likely) into $\mathcal{Z}_{0}$, and to file the state space (and those events equally likely) into $\mathcal{Z}_{1}$. The next drawer we shall populate is $\mathcal{Z}_{\frac{1}{2}}$. This might well be expected: such "even-chance" events play a role in the approach outlined by Ramsey (1931), and their existence is sometimes directly assumed (see for example Gul, 1992). Here, however, we need not make any such assumption; we shall instead construct such an event.

We can already compare two events according to relative likelihood with $\succsim$; let us forget that for a moment. Let us instead consider comparing two events lexicographically:

$$
A>_{L E X} B \text { if and only if } \min A \backslash B<\min B \backslash A .
$$

In other words, when comparing distinct $A$ and $B$, let $s$ be the smallest-index state for which $s$ is in one event but not the other. If $s \in A$, then $A>_{L E X} B$, else $B>_{L E X} A$.

It is well-known that there is an order-preserving homeomorphism-let us call it $\Psi$ between

(i) the subsets of the natural numbers ordered lexicographically, and

(ii) the canonical Cantor set (Cantor, 1883) with the standard ordering of reals. ${ }^{24}$

This observation is important because of the intimate relationship between order and topology on the real line - after all, the standard topology is generated by upper and lower contour sets! Indeed, on the real line each compact set has a maximum, and because the Cantor set is bounded, by the Heine-Borel Theorem each of its closed subsets has a maximum. Because of $\Psi$, in our environment every closed collection of events has a lexicographic maximum.

In particular, the collection of events that are no more likely than their complements,

$$
\mathcal{A}^{-} \equiv\{B \in \mathcal{A} \mid(S \backslash B) \succsim B\}
$$

is closed, and therefore has a lexicographic maximum $H$.

Can $H$ have a finite complement? Under $1-A S$, no: if there were some $s=\max (S \backslash H)$, then the event $(H \backslash\{s+1, s+2, \ldots\},) \cup\{s\}$ would be lexicographically greater than $H$, but by $1-A S$ would also belong to $\mathcal{A}^{-}$, contradicting the definition of $H$. Thus $H$ has an infinite complement, and by construction the union of $H$ with any state in $(S \backslash H)$ is

(i) lexicographically greater than $H$, and thus

(ii) outside of $\mathcal{A}^{-}$, and thus

(iii) more likely than its complement.

It follows from a continuity argument that $H$ is just as likely as its complement, and can therefore be properly be filed into drawer $\mathcal{Z}_{\frac{1}{2}}$. In order to use the full power of this observation, we wish to state it when the event we are trying to split in halves is not necessarily all of $S$, but rather might be any collection $A$ with the required structure. We do this using the notion of subspaces:

\footnotetext{
${ }^{24}$ In fact, $\Psi$ has a simple explanation. Consider the standard construction of the Cantor set through iterative removal of middle thirds. A point in the Cantor set is identified through a sequence of 'lefts' and 'rights': at each stage of the construction, we record some smallest interval, and after middle thirds are removed, we record either its left or right subinterval; the point is the unique intersection of this sequence of recorded intervals. For each $A \subseteq \mathbb{N}, \Psi(A)$ chooses 'right' at the $k$ th step if $k \in A$; otherwise it chooses 'left' - in this way a sequence of inclusions and exclusions encodes a sequence of rights and lefts to uniquely determine a point in the Cantor set. By inspection, $\Psi$ is an order-preserving bijection, and it is routine to show that $\Psi$ and its inverse are continuous.
} 
Definition: ${ }^{25}$ For each likelihood space $(S, \mathcal{A}, \succsim)$ and each $A \in \mathcal{A}$, define the $A$-subspace of $(S, \mathcal{A}, \succsim),\left.(S, \mathcal{A}, \succsim)\right|_{A}$, to be the likelihood space $\left(A, \mathcal{A} \cap 2^{A},\left.\succsim\right|_{A}\right)$, where $\left.\succsim\right|_{A}$ is the binary relation on $\mathcal{A} \cap 2^{A}$ defined by:

$$
B \succsim \mid{ }_{A} C \text { if and only if } B, C \in \mathcal{A} \cap 2^{A} \text { and } B \succsim C .
$$

We sometimes abuse notation, writing $\left.\succsim\right|_{A}$ to denote the entire likelihood space $\left.(S, \mathcal{A}, \succsim)\right|_{A}$. Using this notion, we can talk about 'halving' events with the required structure:

First Halving Lemma: If $(S, \mathcal{A}, \succsim)$ is a purely- $\sigma$-catalogued qualitative probability space satisfying monotone continuity, then for each $A \in \mathcal{A}$ such that $A \succ \emptyset$ and $\left.\succsim\right|_{A}$ satisfies $1-A S$, there is $H \subseteq A$ such that $H \sim A \backslash H$ and $A \succ H \succ \emptyset$.

The proof, sketched throughout this section, is in Appendix 4. Thus it is through the Cantor set that we first glimpse cardinality in our ordinal relation.

\subsection{Greedy transforms}

In practice, what must come next is the population of $\mathcal{Z}_{\frac{1}{4}}$, then $\mathcal{Z}_{\frac{1}{8}}$, and so on. For the moment, however, let us set this problem aside and consider another: if we have populated drawers $\mathcal{Z}_{v}$ and $\mathcal{Z}_{v^{\prime}}$, with $v+v^{\prime} \leq 1$, how can we take one event from $\mathcal{Z}_{v}$ and another event from $\mathcal{Z}_{v^{\prime}}$ so that these two events do not intersect?

Remarkably, it turns out that regardless of $v^{\prime}$, we can always succeed if we first 'blindly' take a specific event from $\mathcal{Z}_{v}$. Moreover, this event is specifically the lexicographicmaximum of $\mathcal{Z}_{v}$. Moreover, this event can be constructed with a so-called "greedy" algorithm. This section is dedicated to that algorithm, which turns out to have further use in the construction of the supercabinet.

In order to build intuition, it is helpful to once again anticipate probabilitiesespecially, here, as numbers associated with singleton events. For the sake of analogy, let us think of singleton events as coins and the associated numbers as monetary values. Let us also think of $v$ as a bus fare, and consider the following scenario:

SCEnARIo 1: You would like to take a bus, and in order to do so, you must pay the fare with exact change. The fare is described to you with a sign specifying the number $v$. In your wallet, you have various coins in different denominations. Can you make exact change, and if so, how?

One possible approach is to use the following greedy algorithm: consider your coins, one at a time, in non-increasing order of value. If the coin under consideration puts your running total over the fare, then return it to your wallet; otherwise, place it into your palm. Once you have considered every coin, present the pile in your palm to the bus driver.

\footnotetext{
${ }^{25}$ We emphasize that because $\left.\succsim\right|_{A}$ compares only subevents of $A$, this notion is distinct from conditional qualitative probability (Savage, 1954). We do not use conditional probabilities anywhere in this article, and we postulate nothing about how beliefs change in response to new information.
} 
It is easy to see the greedy algorithm can unnecessarily fail, for example if the fare is $30 \notin$ when you have a quarter and three dimes. ${ }^{26}$ But there is a particular class of problems for which the greedy algorithm always works: when you have a countably infinite collection of coins whose value tends to zero, your total wealth exceeds the fare, and the value of each coin is less than the value of all smaller coins. ${ }^{27}$ Essentially, the algorithm works because there is always enough and never too much: if a coin is skipped, there is always enough to make up for it, and by design, there is never too much in your palm.

For our purposes, this is only the intuition: while the above logic will apply when at last we have probabilities, we do not have them yet. Right now, we only have some populated drawer $\mathcal{Z}_{v}$; there may not be any singletons at all that have yet been filed! Nevertheless, it is possible to modify the analogy to suit our situation by considering another world where coins do not feature numbers, but rather the value of a pile of coins is given by its mass:

SCEnARIO 2: You would like to take a bus, and in order to do so, you must pay the posted fare with exact change. The fare is described to you with a pile of coins $B$ on one arm of a balance. In your wallet, you have various coins. Can you make exact change, and if so, how?

As it turns out, under the analogous ordinal condition for the coins in your wallet, an analogue of the greedy algorithm works. If $A$ represents the coins in your wallet, and $B$ represents the fare, then let $\mathcal{G}^{A}(B)$ represent the outcome of the greedy algorithm:

Definition: If $(S, \mathcal{A}, \succsim)$ is purely- $\sigma$-catalogued, then for each $A \in \mathcal{A}$, we define the greedy transform $\mathcal{G}^{A}: \mathcal{A} \rightarrow \mathcal{A}$ as follows. For each $B \in \mathcal{A}$, define $\mathcal{G}^{A}(B) \subseteq A$ by:

- Define $\mathcal{G}_{0}^{A}(B) \equiv \emptyset$.

- For each $i \in \mathbb{N}$, define

$$
\mathcal{G}_{i}^{A}(B) \equiv\left\{\begin{array}{lr}
\mathcal{G}_{i-1}^{A}(B) \cup\{i\}, & i \in A \text { and } B \succsim \mathcal{G}_{i-1}^{A}(B) \cup\{i\} \\
\mathcal{G}_{i-1}^{A}(B), & \text { else. }
\end{array}\right.
$$

- Clearly $\left(G_{i}^{A}(B)\right) \in \mathcal{A}^{\mathbb{N}}$ is convergent. Define

$$
\mathcal{G}^{A}(B) \equiv \lim \mathcal{G}_{i}^{A}(B) .
$$

By construction and by monotone continuity, $B \succsim \mathcal{G}^{A}(B)$. Provided $A \succsim B$, when is equivalence guaranteed? As promised:

1-AS LEMmA: If $(S, \mathcal{A}, \succsim)$ is a purely- $\sigma$-catalogued qualitative probability space satisfying monotone continuity, then for each $A \in \mathcal{A}$ such that $\left.\succsim\right|_{A}$ satisfies $1-A S$ and each $B \in \mathcal{A}$ such that $A \succsim B, \mathcal{G}^{A}(B) \sim B$.

\footnotetext{
${ }^{26}$ This failure is an implication of a broader principle: determining whether or not you can make exact change is $N P$-complete, even if it is given that all coin values and the fare are integers! This is the subset sum problem, a special case of the knapsack problem; see for example Garey and Johnson (1979).

${ }^{27}$ This observation is due to Kakeya (Kakeya, 1914; Kakeya, 1915), who moreover showed that for any sequence $\left(\mu_{i}\right) \in \mathbb{R}^{\mathbb{N}}$ with finite sum, the collection of subsequence sums is convex if and only if for each $i \in \mathbb{N}, \sum_{j>i} \mu(j) \geq \mu(i)$.
} 
In other words, if $A$ represents your collection of coins, its subspace satisfies $1-A S$, and you have at least enough for the fare, then $\mathcal{G}^{A}(B)$ precisely pays for the fare. The intuition for why this works is the same as before - after all, "always enough" and "never too much" are ordinal statements!

Thus the file clerk can open the populated drawer $\mathcal{Z}_{v}$, remove any event $B$, and set it as the fare. Then, using the greedy algorithm, he can produce $\mathcal{G}^{S}(B)$, which by construction is necessarily the lexicographic-maximum of $\mathcal{Z}_{v}$.

\subsection{Iterative halving and disjoint pairs}

In the last section, we established that under 1- $A S$,

(i) the lexicographic-maximum of a drawer, and

(ii) the greedy transform, with $S$ as the wallet, of any event from that drawer, are equivalent. But why bother? If we simply intend to take the lexicographic-maximum, why spend time on this detour about how it can be constructed?

The reason is that the greedy transforms have further use; in fact, Appendix 3 is dedicated to its properties. To sketch our proof, we will discuss only the most fundamental of these properties: ${ }^{28}$

Greedy Removal Lemma: If $(S, \mathcal{A}, \succsim)$ is a purely- $\sigma$-catalogued qualitative probability space satisfying monotone continuity, then for each $k \in \mathbb{N}$, each $A \in \mathcal{A}$ such that $\left.\succsim\right|_{A}$ satisfies $(k+1)-A S$, and each $B \in \mathcal{A}$,

$$
\left.\succsim\right|_{A \backslash \mathcal{G}^{A}(B)} \text { satisfies } k \text {-AS. }
$$

That is, if (the subspace associated with) $A$ satisfies $(k+1)-A S$, then removing an image of its greedy transform yields a subevent that (has a subspace which) satisfies $k-A S$. As a first corollary, we have:

SeCond Halving Lemma: If $(S, \mathcal{A}, \succsim)$ is a purely- $\sigma$-catalogued qualitative probability space satisfying monotone continuity and $2-A S$, then for each $A \in \mathcal{A}$ such that $A \succ \emptyset$, there are disjoint $H(A), H^{\prime}(A) \in \mathcal{A}$ such that

(1) $A \sim H(A) \cup H^{\prime}(A)$, and

(2) $A \succ H(A) \sim H^{\prime}(A) \succ \emptyset$.

The idea is simple: remove $\mathcal{G}^{S}(S \backslash A)$, then apply the First Halving Lemma. In this way, we can make 'halves' of any event, and therefore populate $\mathcal{Z}_{\frac{1}{4}}, \mathcal{Z}_{\frac{1}{8}}$, and so on. The proof is in Appendix 4.

As a second corollary to the Greedy Removal Lemma, we can construct disjoint pairs. This can be understood in terms of a third bus scenario:

Scenario 3: You would like to take two consecutive buses: the first to the city center, and the second from there to your destination. In order to do so, for each bus, you must pay the posted fare. The first fare is described to you with a pile of coins $B_{1}$ on one arm of a balance on the first bus; the second fare is described with a pile of coins $B_{2}$ on one

${ }^{28}$ In private communication, Vilmos Komornik proved the cardinal analogue of the Greedy Removal Lemma (Komornik, 2015). Theorem KK, which we use in our proof of Theorem 2, is a joint implication of the results of Kakeya and Komornik. 
arm of a balance on the second bus. In your wallet, you have various coins. Can you make exact change, and if so, how?

Suppose you begin with $S$ in your wallet. As before, you can use the greedy transform to make exact change with the pile $\mathcal{G}^{S}\left(B_{1}\right)$. At that point, what remains in your wallet is $S \backslash \mathcal{G}^{S}\left(B_{1}\right)$. By the Greedy Removal Lemma, the required wallet structure for the greedy algorithm to work has been preserved! Thus you can make exact change for the second bus with the pile $\mathcal{G}^{S \backslash \mathcal{G}^{S}\left(B_{1}\right)}\left(B_{2}\right)$. These two payments are disjoint: physically, $\mathcal{G}^{S}\left(B_{1}\right)$ remains on the first bus, while $\mathcal{G}^{S \backslash \mathcal{G}^{S}\left(B_{1}\right)}\left(B_{2}\right)$ remains on the second; there is no intersection between the two.

Thus given populated $\mathcal{Z}_{v}$ and $\mathcal{Z}_{v^{\prime}}$, the file clerk can first take some arbitrary $A \in \mathcal{Z}_{v}$ and $B \in \mathcal{Z}_{v^{\prime}}$. These may intersect; nevertheless, the file clerk can then take $Z_{v}$ to be $\mathcal{G}^{S}(A)$ and take $Z_{v^{\prime}}$ to be $\mathcal{G}^{S \backslash Z_{v}}(B)$, and these do not intersect.

\subsection{Supercabinet construction}

We are now ready to construct the supercabinet. Let us begin with too many drawers: there is not only one labeled $\frac{1}{2}$, but also one labeled $\frac{2}{4}$, another labeled $\frac{16}{32}$, and so on. In general, for each pair $p, q \in\{0,1,2, \ldots\}$ with $p \leq 2^{q}$, there is a drawer labeled $\frac{p}{2^{q}}$. We let $\mathcal{Z}_{q}^{p}$ denote the drawer labeled $\frac{p}{2^{q}}$, and once $\mathcal{Z}_{q}^{p}$ is populated, we let $A_{q}^{p}$ denote its lexicographic-maximum.

First, we fill the drawers of the form $\frac{1}{2^{q}}$ through iterative applications of the Second Halving Lemma. Then, for each $q$, we fill the drawers of the form $\frac{p}{2^{q}}$ through iterative disjoint union involving $A_{q}^{1}$. To be more explicit about this latter operation: given $A_{q}^{p}$, we define

$$
A_{q}^{p+1} \equiv \mathcal{G}^{S}\left(A_{q}^{p} \cup \mathcal{G}^{S \backslash A_{q}^{p}}\left(A_{q}^{1}\right)\right)
$$

This process can be understood as the construction of a sequence of progressively finer rulers (Figure 3).

At this point, with $2-A S$, it is possible to show that the extra drawers are in fact redundant: if two drawers bear labels that share a reduced fraction, then their contents are the same. So should we discard all the redundant drawers - say, keeping only those whose labels are reduced fractions - to yield our supercabinet? Not yet; the problem is that while this would yield properly-painted drawers that are populated, we do not yet know that this structure would indeed be supercabinet. In particular, we know that if we give the file clerk $v$ and $v^{\prime}$ with $v+v^{\prime} \leq 1$, he can locate $Z_{v}$ in $\mathcal{Z}_{v}$ and $Z_{v^{\prime}}$ in $\mathcal{Z}_{v^{\prime}}$ such that $Z_{v}$ and $Z_{v^{\prime}}$ are disjoint, but we do not know that he can locate $Z_{v} \cup Z_{v^{\prime}}$ in $\mathcal{Z}_{v+v^{\prime}}$.

Toward this end, it turns out that it is convenient to continue with the redundant drawers in order to emphasize the different constructions that lead to the same equivalence class. In particular, for each $q \in\{0,1, \ldots\}$ and each pair $p, p^{\prime} \in\left\{0,1, \ldots, 2^{q}\right\}$ such that $p+p^{\prime} \leq 2^{q}$, define:

$$
A_{q}^{p} \biguplus A_{q}^{p^{\prime}} \equiv \mathcal{G}^{S}\left(A_{q}^{p} \cup \mathcal{G}^{S \backslash A_{q}^{p}}\left(A_{q}^{p^{\prime}}\right)\right)
$$

In order to prove that this binary operation in fact captures addition - that $A_{q}^{p} \biguplus A_{q}^{p^{\prime}}=$ $A_{q}^{p+p^{\prime}}$ —we follow Peano's axiomatization of addition (Peano, 1889). The essential step of the inductive argument is establishing that $A_{q}^{p+p^{\prime}}=A_{q}^{p} \biguplus A_{q}^{p^{\prime}}$ implies $A_{q}^{p+\left(p^{\prime}+1\right)}=$ 


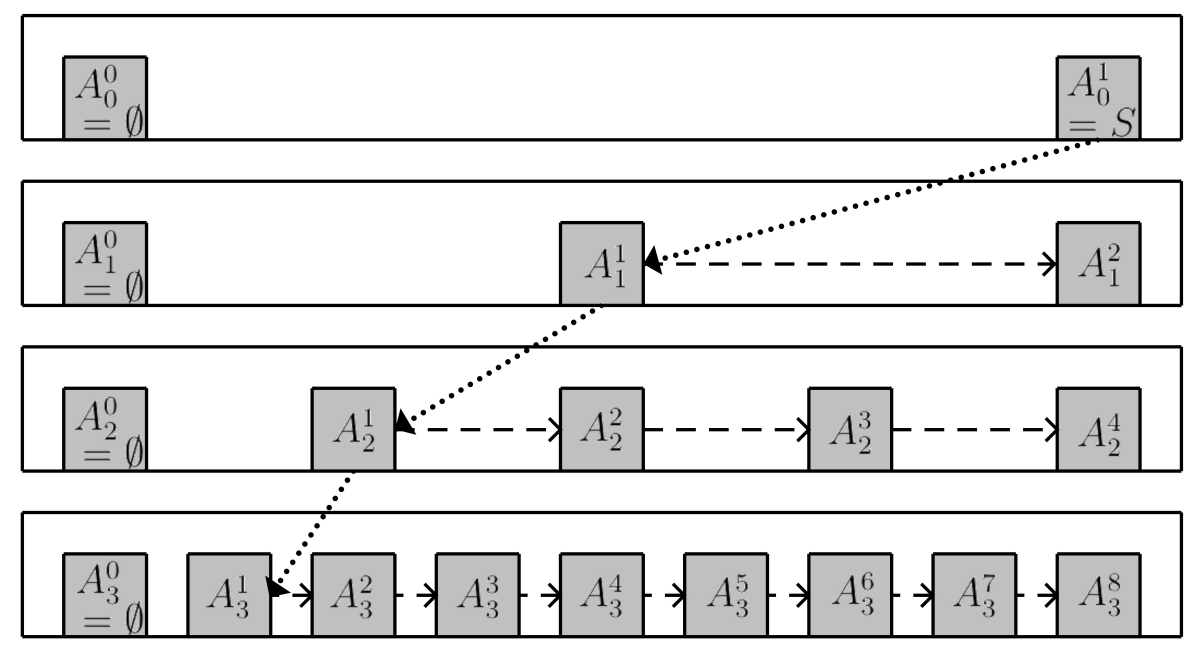

Figure 3: Each row is one member in our sequence of progressively finer rulers. The leftmost point on each is identified by $\emptyset$. The diagonal dotted lines represent construction through "halving." The horizontal dashed lines represent construction through "addition by one." It can be proved that any two events aligned vertically are in fact the same event.

$A_{q}^{p} \biguplus A_{q}^{p^{\prime}+1}$. It is here that we require 3 - $A S$ : in general, $\left|\left\{p, p^{\prime}, 1\right\}\right|=3$. It is in this way that we can deduce cancellation instead of assuming it, and prove:

Supercabinet Construction Lemma: If $(S, \mathcal{A}, \succsim)$ is purely- $\sigma$-catalogued and satisfies $3-A S$, then $(S, \mathcal{A}, \succsim)$ has a supercabinet.

For details, and to see that the other supercabinet properties can be verified, see Appendix 5. Together with the Supercabinet Blueprint Lemma, we have at once:

Theorem 1: If $(S, \mathcal{A}, \succsim)$ is a purely- $\sigma$-catalogued qualitative probability space satisfying monotone continuity and 3 - $A S$, then it has a unique representation $\mu \in \mathbb{M}^{\sigma}(\mathcal{A})$.

The formal proof is in Appendix 5.

\section{Proof sketch for Theorem 2}

Next, we turn our attention to finitely-catalogued qualitative probability spaces. As with Theorem 1, we proceed by constructing a supercabinet.

Recall that $S^{\oplus}$ gathers the states whose singletons are the cores; $S^{\circ}$ gathers the other states; and $\mathcal{A}^{\circ}$ is the collection of events contained in $S^{\circ}$, which includes no atoms. Our approach is to apply the Supercabinet Construction Lemma to a purely- $\sigma$-catalogued space which is derived from our finitely-catalogued space, essentially by partitioning $S^{\circ}$ into events that we temporarily pretend are cores.

To begin, finitely-catalogued spaces involve events that contain no atoms, which it is helpful to think of as cloth-like fabric that may be cut into smaller pieces of any specification. Indeed, by Theorem $\mathrm{V} 2,\left.\succsim\right|_{S^{\circ}}$ has an infinitely-divisible measure representation 
$\mu^{\circ}$, and moreover, this intuition is even harmonious with atoms:

Carving Lemma: If $(S, \mathcal{A}, \succsim)$ is a catalogued qualitative probability space satisfying monotone continuity, then for each pair $A, B \in \mathcal{A}$ such that $A \in \mathcal{A}^{\circ}$ and $A \succsim B$, there is $B^{\prime} \subseteq A$ such that $B^{\prime} \in \mathcal{A}$ and $B^{\prime} \sim B$.

The proof, which unsurprisingly involves retracing some arguments from Villegas (1964), is in Appendix 6.

How do we partition $S^{\circ}$ ? Before we begin, let us call $S^{\circ}$ the fabric. At each step, we cut off a piece of the fabric and reclassify it as an honorary core, and after an infinite sequence of steps we finish and are out of fabric. Because it is convenient to work with $\mu^{\circ}$, we first use the Carving Lemma and Theorem V2 to cut off an arbitrary non-null event that is less likely than the least-likely core. By $3-A S$, this first honorary core is less likely than $S^{\circ}$ as well, and we are therefore left with some (non-null) fabric. We then proceed recursively.

At the start of each step, some (non-null) fabric remains. Let $A$ be the least-likely of the actual and honorary cores, which in fact will always be honorary. We use Theorem V2 to partition what remains of the fabric into four equally likely events: $B_{1}, B_{2}, B_{3}$, and $B_{4}$. If $A \succsim B_{1}$, then we cut off $B_{1}$; otherwise, we use the Carving Lemma to cut off an event that is as likely as $A_{i}$. In both cases, fabric indeed remains after the cut.

Appealing to $\mu^{\circ}$, it is clear that we first cut off some finite number of pieces that have the same measure as the first honorary core, after which point each new honorary core is less likely than its predecessors. By construction, each of these latter honorary cores has a measure equal to three times the sum of the measures of its successors. Put another way, beyond a certain point, the measures of the honorary cores are geometric: if one has measure $v$, then the next has measure $\frac{3 v}{4}$.

We now have a coarser $\sigma$-algebra $\mathcal{A}^{\prime} \subseteq \mathcal{A}$ : the cores, the honorary cores, and their unions. By taking the restriction of $\succsim$ to $\mathcal{A}^{\prime}$ and relabeling the singletons, we construct a finitely-catalogued qualitative probability space that (we verify) satisfies $3-A S$. At this point, we apply the Supercabinet Construction Lemma to produce a supercabinet, and in doing so reveal a supercabinet for the original space. By the Supercabinet Blueprint Lemma, we have established:

TheOREM 2: If $(S, \mathcal{A}, \succsim)$ is a finitely-catalogued qualitative probability space satisfying monotone continuity and 3 - $A S$, then it has a unique representation $\mu \in \mathbb{M}^{\sigma}(\mathcal{A})$.

The formal proof is in Appendix 6.

\section{$5 \quad$ Proof sketch for Theorem 4}

\subsection{Splitting the atoms}

At last, we are ready to consider $\sigma$-catalogued qualitative probability spaces, where there is some messy sequence of cores $S^{\oplus}$ together with some infinitely-divisible fabric $S^{\circ}$. Essentially, there are two major steps: (i) construct $\mu$, and (ii) verify that it is a representation. In a way, these steps are intertwined: we do not construct $\mu$ as directly as possible, but rather take an indirect path that reveals much of what $\mu$ represents. This section is dedicated to describing this indirect path at a high level. 
The fundamental technique in this construction is splitting the atoms into a left space and a right space. In particular, imagine that the cores are arranged along a line from left to right in non-increasing order of likelihood. In order to split the atoms, simply pick an adjacent pair of cores and build a wall between them. So long as this wall is in place, the left atoms are segregated from the right: we will consider the left cores with the fabric, and separately we will consider the right cores with the fabric, but never will we consider the left and right cores together. Once we are done, we may tear down that wall and build another elsewhere, but at any given time there will always be at most one wall.

Formally, for each 'middle' $m \in S^{\oplus}$, we define two qualitative probability spaces, one for the left and the other for the right:

- Define $S^{\leftarrow m} \equiv\{1,2, \ldots, m\} \cup S^{\circ}$; define $\mathcal{A}^{\leftarrow m}$ to be the events contained in $S^{\leftarrow m}$; and let $\succsim \leftarrow m$ be the associated restriction of $\succsim$.

- Define $S^{m \rightarrow} \equiv\{m, m+1, \ldots\} \cup S^{\circ}$; define $\mathcal{A}^{m \rightarrow}$ to be the events contained in $S^{m \rightarrow}$; and let $\succsim^{m \rightarrow}$ be the associated restriction of $\succsim$.

In the informal description, building a wall means separately considering $S^{\leftarrow m}$ and $S^{m+1 \rightarrow}$ (and their associated spaces).

Our first order of business is to establish that far enough in the tail - that is, for sufficiently high $m$ - the left and right spaces each have a unique measure representation. For such a pair $S^{\leftarrow m}$ and $S^{m+1 \rightarrow}$, we can then stitch the two measures together, thus constructing a grand measure $\mu^{m}$ over the entire space $\mathcal{A}$ that represents both the left and right spaces. Finally, we establish that all of these grand measures coincide, and we take this common measure as $\mu$, completing the construction. (Note that there is then still more to be done, as it is not enough for a measure to represent all comparisons where both events come from the same left or right space deep in the tail.)

\subsection{Deep left spaces}

We first show that each sufficiently deep left space has a unique measure representation. One might view all of our work thus far as culminating in this fact: the left spaces are finitely-catalogued, and we will therefore apply Theorem 2, whose proof required the construction of a supercabinet. However, we can not apply Theorem 2 just yet: while left spaces are finitely-catalogued, they need not satisfy $3-A S$.

In fact, while it is true (and we will establish) that sufficiently deep left spaces do satisfy $3-A S$ when the grand space satisfies $3^{+}-A S$, this is not necessarily so when the grand space only satisfies $3-A S$. Indeed, suppose the most-likely core $\{1\}$ is such that $S \backslash\{1\}$ can be partitioned into three events that are each precisely as likely as $\{1\}$. In this case, each left space includes $\{1\}$, yet discards some (non-null) cores and thus cannot satisfy $3-A S$.

As promised, this is no problem when the grand space satisfies $3^{+}-A S$. For each $s \in S^{\oplus}$, let us say that $t \geq s$ is a truncation point of $s$ if, informally, even after truncating the tail beyond $t,\{s\}$ is third-order swarmed by less-likely non-null events. By a continuity argument, we can construct for each $s$ a particular truncation point $\tau(s)$. Moreover, by another continuity argument, deep enough in the tail each $s$ is its own truncation point, as eventually the cores become smaller than 'thirds' of the fabric. Letting $s^{*} \in S^{\oplus}$ denote the earliest $s$ which is its own truncation point, we define

$$
m^{\leftarrow *} \equiv \max \left\{\tau(1), \tau(2), \ldots, \tau\left(s^{*}-1\right), \tau\left(s^{*}\right)\right\} .
$$


By construction, if $m \geq m^{\leftarrow *}$, then $\left(S^{\leftarrow m}, \mathcal{A}^{\leftarrow m}, \succsim^{\leftarrow m}\right)$ satisfies $3-A S$, and thus by Theorem 2 has a unique representation $\mu^{\leftarrow m}$. Altogether, each sufficiently deep left space has a unique measure representation.

\subsection{Deep right spaces}

While each left space has a finite number of cores and some fabric, and some of them satisfy the requirements of Theorem 2, this is not the case for the right spaces. Each right space is a messy sequence of cores together with some fabric, and in this way is like the grand space.

That said, in deeper right spaces the relative likelihood of the cores is smaller, and in sufficiently deep right spaces the cores are less likely than the fabric. It turns out that this is sufficient for our purposes:

TheOREM 3: If $(S, \mathcal{A}, \succsim)$ is a catalogued qualitative probability space satisfying monotone continuity and unlikely cores, then it has a unique representation $\mu \in \mathbb{M}^{\sigma}(\mathcal{A})$.

While this result is of independent interest, for the purposes of this section Theorem 3 is simply a lemma for Theorem 4. It turns out that the smaller proof-within-a-proof has the same structure as the larger proof - construct the measure, then verify it is a representation - and moreover, the smaller proof's verification step is a simpler version of the larger proof's.

The construction of the measure is simple: use Theorem V2 to construct a representation of the fabric; use the Carving Lemma to associate each union of cores with an equally likely fabric event; assign to each union of cores the measure of its associate; complete the measure and normalize.

For verification, the idea is that for each pair of events $A$ and $B$, we use a particular algorithm to construct a list of pairs $(A, B)=\left(A_{1}, B_{1}\right),\left(A_{2}, B_{2}\right),\left(A_{3}, B_{3}\right), \ldots$ such that for each $i$, we can establish:

(i) $A_{i} \succsim B_{i}$ if and only if $A_{i+1} \succsim B_{i+1}$, and

(ii) $\mu\left(A_{i}\right) \geq \mu\left(B_{i}\right)$ if and only if $\mu\left(A_{i+1}\right) \geq \mu\left(B_{i+1}\right)$.

Moreover, the algorithm is guaranteed to terminate with a pair $\left(A_{\Omega}, B_{\Omega}\right)$ for which we can prove the statement " $A_{\Omega} \succsim B_{\Omega}$ if and only if $\mu\left(A_{\Omega}\right) \geq \mu\left(B_{\Omega}\right)$." Visually, it can be helpful to imagine a "logical ladder" whose rails and rungs are made of if-and-only-if arrows.

How does this algorithm work? At each step $i$, let $n_{i}^{\circ} \in\{0,1,2\}$ count how many of $A_{i}$ and $B_{i}$ intersect $S^{\circ}$, and similarly let $n_{i}^{\oplus} \in\{0,1,2\}$ count how many of $A_{i}$ and $B_{i}$ intersect $S^{\oplus}$. We proceed by performing the operation for the earliest of these cases which applies:

1. If $A_{i}=\emptyset$ or $B_{i}=\emptyset$, then it is easy to show that we are done.

2. If either count is zero, then we are either comparing two fabric events or two unions of cores, and it is easy to show that we are done.

3. If $n_{i}^{\circ}=2$, then we can use the Carving Lemma to replace the smaller fabric component with one that is contained within the larger, then subtract this from both. We begin the next step with $n_{i+1}^{\circ}<2$ and $n_{i+1}^{\oplus}=n_{i}^{\oplus}$. 
4. If $n_{i}^{\circ}=1$ and $n_{i}^{\oplus}=2$, then one of the events is a union of cores. Because the fabric is more likely than the cores, by the Carving Lemma, we can replace the union of cores with an equally-likely fabric event. We begin the next step with $n_{i}^{\circ}=2$ and $n_{i+1}^{\oplus}=1$.

5. If $n_{i}^{\circ}=1$ and $n_{i}^{\oplus}=1$, and neither $A_{i}$ nor $B_{i}$ is $\emptyset$, then as in the previous case, we can replace the union of cores with an equally-likely fabric event. We begin the next step with $n_{i}^{\circ}=2$ and $n_{i+1}^{\oplus}=0$.

It is clear that this process must terminate. For details, see Appendix 7.

With Theorem 3 established, we return to the deep right spaces in our proof of Theorem 4. Indeed, we simply define

$$
m^{* \rightarrow} \equiv \min \left\{s \in S^{\oplus} \mid S^{\circ} \succsim\{s, s+1, \ldots\}\right\},
$$

which is well-defined by a continuity argument. By construction, if $m \geq m^{* \rightarrow}$, then $\left(S^{m \rightarrow}, \mathcal{A}^{m \rightarrow}, \succsim^{m \rightarrow}\right)$ satisfies unlikely cores, and thus by Theorem 3 has a unique representation $\mu^{m \rightarrow}$. Altogether, each sufficiently deep right space has a unique measure representation.

\subsection{Constructing the measure}

In this section, we construct $\mu$ not once or twice, but an infinite number of times. In particular, for each sufficiently deep $m$, we use the results of the previous sections to construct a unique measure $\mu^{m}$ that represents both the associated left space and the associated right space. We then show that all of these measures are in fact the same measure, which we call $\mu$.

To begin, define $m^{*} \equiv \max \left\{m^{\leftarrow *}, m^{* \rightarrow}\right\}$, take $m \geq m^{*}$, and split the atoms by building a wall between $m$ and $m+1$. By the previous sections, we have unique representations $\mu^{\leftarrow m}$ and $\mu^{m+1 \rightarrow}$ for the left space and the right space, respectively. To build a grand measure $\mu^{m}$ such that

(i) the restriction to the left space is a positive multiple of $\mu^{\leftarrow m}$, and

(ii) the restriction to the right space is a positive multiple of $\mu^{m+1 \rightarrow}$,

it suffices to simply solve a system of equations to determine $\mu^{m}\left(S^{\circ}\right), \mu^{m}(\{1,2, \ldots, m\})$, and $\mu^{m}\left(\{m+1, m+2, \ldots\}\right.$, then complete the measure. Altogether, $\mu^{m}$ exists as the unique measure on $\mathcal{A}$ that represents both comparisons for pairs in $\mathcal{A}^{\leftarrow m}$ and for pairs in $\mathcal{A}^{m+1 \rightarrow}$.

We claim that for each pair $m, m^{\prime} \geq m^{*}, \mu^{m}=\mu^{m^{\prime}}$. It suffices to prove that for each $m \geq m^{*}, \mu^{m}=\mu^{m+1}$, and due to the uniqueness of each measure, it suffices to prove that $\mu^{m+1}$ represents both comparisons for pairs in $\mathcal{A}^{\leftarrow m}$ and for pairs in $\mathcal{A}^{m+1 \rightarrow}$. Representation for the former comparisons is immediate as $\mathcal{A}^{\leftarrow m} \subseteq \mathcal{A}^{\leftarrow m+1}$, so we need only establish representation for comparisons of pairs in $\mathcal{A}^{m+1 \rightarrow}$. The argument involves establishing that normalizations of $\mu^{m}$ and $\mu^{m+1}$ restricted to $\mathcal{A}^{m+1 \rightarrow}$ are both measure representations when further restricted to $\mathcal{A}^{m+2 \rightarrow}$, and thus agree everywhere but $\{m+1\}$; and then also there as well, since by the Carving Lemma $\{m+1\}$ has the same measure as some equally-likely fabric event. For details, see Step 6 in Appendix 8.

Altogether, we have a measure $\mu$ on $\mathcal{A}$ that represents all sufficiently deep left spaces and all sufficiently deep right spaces. At this point, all that remains is to verify that $\mu$ in fact represents all comparisons. 


\subsection{Verification of representation}

Again, we proceed as we did in the proof of Theorem 3 to verify representation. In particular: again, we take an arbitrary pair of events $A$ and $B$ and construct a "logical ladder" of if-and-only-if arrows. Again, by algorithm, we construct a list of pairs $(A, B)=$ $\left(A_{1}, B_{1}\right),\left(A_{2}, B_{2}\right),\left(A_{3}, B_{3}\right), \ldots$ such that for each $i$, we can establish:

(i) $A_{i} \succsim B_{i}$ if and only if $A_{i+1} \succsim B_{i+1}$, and

(ii) $\mu\left(A_{i}\right) \geq \mu\left(B_{i}\right)$ if and only if $\mu\left(A_{i+1}\right) \geq \mu\left(B_{i+1}\right)$.

Again, the algorithm is guaranteed to terminate with a pair $\left(A_{\Omega}, B_{\Omega}\right)$ for which we can prove the statement " $A_{\Omega} \succsim B_{\Omega}$ if and only if $\mu\left(A_{\Omega}\right) \geq \mu\left(B_{\Omega}\right)$." But this time, the algorithm involves a partition of the state space into three parts instead of two.

Before we begin, we require one final technique. For a given core $\{s\}$, we would like to construct a finite twin: an equally-likely event which, if it includes any cores, only includes a finite number of less-likely ones. If the fabric alone is at least as likely as $\{s\}$, then we can do so by the Carving Lemma. In fact, even if the fabric and less-likely cores are together more likely than $\{s\}$, we can succeed-provided the fabric is at least as likely as the less-likely cores:

Finite-Twin Lemma: If $(S, \mathcal{A}, \succsim)$ is a $\sigma$-catalogued qualitative probability space satisfying monotone continuity and $1^{+}-A S$, then for each $s \in S^{\oplus}$ such that $S^{\circ} \succsim\{s+1, s+2, \ldots\}$, there is $T_{s} \in \mathcal{A}$ such that

(i) $T_{s} \subseteq\{s+1, s+2, \ldots\} \cup S^{\circ}$,

(ii) $T_{s} \cap S^{\oplus}$ is finite, and

(iii) $T_{s} \sim\{s\}$.

How does the algorithm work? To begin, we partition $S$ into $S^{\circ}, S^{\leftarrow} \equiv\left\{1,2, \ldots, m^{*}\right\}$, and $S^{\rightarrow} \equiv\left\{m^{*}+1, m^{*}+2, \ldots\right\}$. Let us say that each event $A \in \mathcal{A}$ has three components: its left component $A \cap S^{\leftarrow}$, its right component $A \cap S^{\rightarrow}$, and its fabric component $A \cap S^{\circ}$.

At each step $i$, we count components. More precisely, let $n_{i}^{\circ} \in\{0,1,2\}$ count how many of $A_{i}$ and $B_{i}$ intersect $S^{\circ}$, let $n^{\leftarrow} \in\{0,1,2\}$ count how many of $A_{i}$ and $B_{i}$ intersect $S^{\leftarrow}$, and let $n_{i} \in\{0,1,2\}$ count how many of $A_{i}$ and $B_{i}$ intersect $S^{\rightarrow}$. We proceed by performing the operation for the earliest of these cases which applies:

1. If $A_{i}=\emptyset$ or $B_{i}=\emptyset$, then it is easy to show that we are done.

2. If any count is zero, we are done. This is immediate if both $A_{i}$ and $B_{i}$ belong to the same left space or the same right space, while if both are contained in $S^{\oplus}$ this can be established by using the Carving Lemma to exchange each event's right component with an equally-likely fabric component, leaving us with two events in the same left space.

3. If $n_{i}^{\circ}=2$, then we can use the Carving Lemma to replace the smaller fabric component with one that is contained within the larger, then subtract this from both. We begin the next step with $n_{i+1}^{\circ}<2$ and $n_{i+1}=n_{i}$.

4. If $n_{i}^{\circ}=1$ and $n_{i}=2$, then one of the events has no fabric component. Because the fabric is more likely than the cores, by the Carving Lemma, we can replace this event's right component with an equally-likely fabric component. We begin the next step with $n_{i}^{\circ}=2$ and $\overrightarrow{n_{i+1}}=1$. 
5. If $n_{i}^{\circ}=1$ and $n^{\rightarrow}=1$, and neither event is only a left component, then as in the previous case, there is an event with no fabric component, and we can replace its right component with an equally-likely fabric component. We begin the next step with $n_{i}^{\circ}=2$ and $\overrightarrow{n_{i+1}}=0$.

6. If $n_{i}^{\circ}=1$ and $n^{\rightarrow}=1$, and one of the events is only a left component but neither event is $\emptyset$, then at this point we modify the left-only event. In particular, we use the Finite-Twin Lemma to replace the highest-index core with its finite twin. How do we know that $\mu$ assigns both twins the same measure? We know this because while the finite twin may extend far past $m^{*}$ into $S^{\rightarrow}$, nevertheless, there is some deeper left space which both twins share.

It is straightforward to verify that we cannot indefinitely loop through the first four instructions, while immediately after the fifth the algorithm will terminate. Thus in order for the algorithm to loop forever, the sixth operation must be performed an infinite number of times. But this is impossible: each time the sixth operation is performed, one of the two left components becomes lexicographically-smaller; and none of the other operations alter left components; thus because $\mathcal{A}^{\leftarrow}$ is finite, the process must terminate.

This completes our verification that $\mu$ is in fact a measure; for details, see Appendix 8. Altogether, we have established:

THEOREM 4: If $(S, \mathcal{A}, \succsim)$ is a $\sigma$-catalogued qualitative probability space satisfying monotone continuity and $3^{+}-A S$, then it has a unique representation $\mu \in \mathbb{M}^{\sigma}(\mathcal{A})$.

\section{Proof sketch for Theorem 5}

Our final objective is to establish that, as promised, there has been essentially no loss of generality in focusing on catalogued spaces where each atom has a singleton core. This last step is relatively simple: we select some pairwise-disjoint collection of atoms and remove them, and do so such that there are no atoms in what remains. In general, there is not a unique such collection, but the selection does not matter for our purposes. Using an argument of Villegas (1964) (though not his related result, which has a minor mistake $\left.{ }^{29}\right)$, this collection is at most countably infinite. When we 'pretend' that they are cores, we can apply Theorem 1, Theorem 2, or Theorem 4; for details, see Appendix 9. This completes the proof of our main result:

TheOREM 5: A triple $(S, \mathcal{A}, \succsim)$ is a qualitative probability space satisfying monotone continuity and $3^{+}-A S$ if and only if it has a unique representation $\mu \in \mathbb{M}^{\sigma}(\mathcal{A})$ such that for each $A \in \mathcal{A}$,

(i) if $A$ is an atom, $\mu(\cup(\downarrow A))>3 \mu(A)>0$, and

(ii) if $A$ contains no atoms, for each $\lambda \in[0,1]$, there is $B \subseteq A$ such that $\mu(B)=\lambda \mu(A)$.

\footnotetext{
${ }^{29}$ Lemma 4 of Villegas (1964) incorrectly claims that for each monotonely continuous qualitative probability, the collection of atoms is at most countably infinite. As a counterexample, consider $S=[0,1]$ and the (monotonely continuous) qualitative probability on $2^{S}$ for which $\{0\} \sim S$ : each event that includes 0 is an atom. To address this, we simply borrow the sound arguments and replace the faulty ones.
} 


\section{Conclusion}

Our main results do not require one to interpret $\mathcal{A}$ as a collection of events and $\succsim$ as beliefs; we discuss two alternative interpretations here.

\section{Preferences over parts of a heterogeneous good}

What sort of economic model allows us to classify an infinitely-divisible good as either homogeneous or heterogeneous? One approach is to consider a collection of pieces into which the good may be divided, and associate each agent with a personal measure over these pieces his preferences respect. When all agents share a measure - such as gallons when the good is milk - the good is homogeneous. When the agents' measures differ, the good is instead heterogeneous.

The classic problem of fair division, starting with Steinhaus (1948), is the problem of partitioning a heterogeneous good (or a cake) into parts (or slices) and then assigning those parts to agents according to some notion of fairness. Similarly, the model of land for urban economics proposed by Berliant (1985) treats land as a heterogeneous good that can be divided into parcels; this model has also been used in the context of fair division (Berliant, Thomson, and Dunz, 1992). The standard assumption in both settings is that each agent's preferences are represented by a measure, and the use of atomless measures has axiomatic foundations for both preferences over slices (Barbanel and Taylor, 1995) and preferences over parcels (Berliant, 1986). Our theorem is also a preference representation theorem for these settings, differing from the existing results in that it allows atoms: crumbs in the context of cake-cutting, ${ }^{30}$ or parcels that cannot be subdivided (such as perhaps cities or houses) in the context of land.

\section{Intertemporal preferences}

The standard model of intertemporal preference is a special case of the Savage model with an alternative interpretation of its primitives: $S=\mathbb{N}$, with its usual well-ordering, the members of $S$ interpreted as periods. This literature studies preferences of an agent (or dynasty, or institution) over consumption streams, and the preferences with $\sigma$-measure subjective expected utility representations are precisely, in the language of Olea and Strzalecki (2014), the time separable class. This central class contains:

- geometric discounting, (Samuelson, 1937; Koopmans, 1960; Koopmans, 1972; Bleichrodt, Rohde, and Wakker, 2008),

- generalized hyperbolic discounting (Loewenstein and Prelec, 1992), and

- quasi-hyperbolic discounting (Laibson, 1997; Olea and Strzalecki, 2014).

Because this class uses atoms, the Savage (1954) characterization of subjective expected utility does not apply. The standard approach in the literature is to take preferences over consumption streams with a rich consumption space, such as Euclidean commodity space.

By contrast, our theorem applies in the minimal case that commodity space has two members, allowing us to handle the case where consumption in each period consists of some indivisible goods (or objects). This setting is also considered by Kochov (2013), who

\footnotetext{
${ }^{30} \mathrm{An}$ example of such a crumb is the small plastic figurine (la fève) in a French king cake. According to tradition, whomever receives it with his slice is king for the day.
} 
proposes patience: consumption of the better object on dates $\{2,3, \ldots\}$ is preferred to consumption of the better object on date $\{1\}$. Kochov establishes that if $\succsim$ has a geometric representation and satisfies patience, then $\succsim$ has no other geometric representation; a corollary is that a geometric representation is unique under 1-AS.

Our atom-swarming axioms may be interpreted as stronger patience requirementsafter all, each atom is a date, and for each date the less-likely non-null events (usually) are gathered by the dates that follow; thus these axioms (usually) require that at any point in time the future sufficiently overwhelms the present. In the special case that beliefs admit a geometric representation, $3^{+}-A S$ is satisfied if and only the discount factor is greater than $\frac{3}{4}$.

\section{Appendix 1}

In this appendix, we state and prove (or provide a proof reference for) four basic lemmas about qualitative probabilities: the Complement Lemma, the Domination Lemma, the Continuity Lemma, and the Limit-Order Lemma.

The Complement Lemma states that order reverses under complements. This is a slight extension of exercise 3 on page 32 of Savage (1972), and thus the proof is omitted.

- Complement Lemma (SAvage, 1954): If $(S, \mathcal{A}, \succsim)$ is a qualitative probability space, then for each $A \in \mathcal{A}$ and each pair $B, B^{\prime} \subseteq A, B \succsim B^{\prime}$ if and only if $A \backslash B^{\prime} \succsim A \backslash B$.

The Domination Lemma states that for any two pairs such that the first is disjoint and dominates the second in likelihood, the union of the first is at least as likely as the union of the second. Moreover, strict domination implies the union of the first is more likely than the union of the second. This is a slight extension of exercise $5 \mathrm{a}$ on page 32 of Savage (1972), and thus the proof is omitted.

- Domination Lemma (Savage, 1954): If $(S, \mathcal{A}, \succsim)$ is a qualitative probability space, then for each four $A, A^{\prime}, B, B^{\prime} \in \mathcal{A}$, if

(1) $A \cap A^{\prime}=\emptyset$,

(2) $A \succsim B$, and

(3) $A^{\prime} \succsim B^{\prime}$

then $A \cup A^{\prime} \succsim B \cup B^{\prime}$. Moreover, if $A \succ B$, then $A \cup A^{\prime} \succ B \cup B^{\prime}$.

The Continuity Lemma involves pointwise convergence, its associated topology (under which $\mathcal{A}$ is closed), and the standard requirement that upper and lower contour sets are closed:

Definition: An event sequence $\left(A_{i}\right) \in \mathcal{A}^{\mathbb{N}}$ is convergent if for each $s \in S$, either

(1) there is $i^{*} \in \mathbb{N}$ such that for each $i \geq i^{*}, s \in A_{i}$, and

(2) there is $i^{*} \in \mathbb{N}$ such that for each $i \geq i^{*}, s \notin A_{i}$.

In this case, we say $\cup_{i=1}^{\infty}\left(\cap_{j=i}^{\infty} A_{j}\right)=\cap_{i=1}^{\infty}\left(\cup_{j=i}^{\infty} A_{j}\right)$ is the (pointwise) limit of $\left(A_{i}\right)$, written $\lim A_{i}$.

○ Continuity: For each $A \in \mathcal{A}$ and each convergent $\left(B_{i}\right) \in \mathcal{A}^{\mathbb{N}}$,

(1) if for each $i \in \mathbb{N}, B_{i} \succsim A$, then $\lim B_{i} \succsim A$, and 
(2) if for each $i \in \mathbb{N}, A \succsim B_{i}$, then $A \succsim \lim B_{i}$. :

Though continuity seems stronger than monotone continuity, in fact the two are equivalent for qualitative probabilities, a point which to our knowledge has not been made previously. Based on this equivalence, we are justified in writing (monotone) continuity in stating our results while using continuity in our proofs:

- Continuity Lemma: If $(S, \mathcal{A}, \succsim)$ is a qualitative probability space, then it satisfies monotone continuity if and only if it satisfies continuity.

Proof: Because for each sequence $\left(B_{i}\right) \in \mathcal{A}^{\mathbb{N}}$ such that $B_{1} \supseteq B_{2} \supseteq \ldots, \cap B_{i}=\lim B_{i}$, clearly continuity implies monotone continuity. Suppose $\succsim$ is a qualitative probability satisfying monotone continuity.

To see that upper contour sets are closed, let $A \in \mathcal{A}$ and let $\left(B_{i}\right) \in \mathcal{A}^{\mathbb{N}}$ be convergent such that for each $i \in \mathbb{N}, B_{i} \succsim A$. Define $B_{\infty} \equiv \lim B_{i}$. For each $i \in \mathbb{N}$, define $C_{i} \equiv \cup_{j \geq i} B_{j}$. By construction, $C_{1} \supseteq C_{2} \supseteq \ldots$, and by monotonicity, for each $i \in \mathbb{N}$, $C_{i} \succsim B_{i} \succsim A$; thus by monotone continuity, $\cap C_{i} \succsim A$.

We claim $\cap C_{i}=B_{\infty}$. Indeed, let $s \in B_{\infty}$. Then there is $i \in \mathbb{N}$ such that $j \geq i$ implies $s \in B_{j}$; thus for each $i \in \mathbb{N}, s \in C_{i}$, so $s \in \cap C_{i}$. Conversely, let $s \in S \backslash B_{\infty}$. Then there is $i \in \mathbb{N}$ such that $j \geq i$ implies $s \notin B_{j}$; thus $s \notin C_{i}$, so $s \notin \cap C_{i}$. Thus $B_{\infty}=\cap C_{i}$, so $B_{\infty} \succsim A$, as desired.

To see that lower contour sets are closed, let $A \in \mathcal{A}$ and let $\left(B_{i}\right) \in \mathcal{A}^{\mathbb{N}}$ be convergent such that for each $i \in \mathbb{N}, A \succsim B_{i}$. Define $B_{\infty}=\lim B_{i}$. Then $\left(S \backslash B_{\infty}\right)=\lim \left(S \backslash B_{i}\right)$. By the Complement Lemma, for each $i \in \mathbb{N},\left(S \backslash B_{i}\right) \succsim(S \backslash A)$. As upper contour sets are closed, $\left(S \backslash B_{\infty}\right) \succsim(S \backslash A)$, so by the Complement Lemma, $A \succsim B_{\infty}$, as desired.

The Limit-Order Lemma states that for each pair of convergent sequences, if each member of the first sequence is at least the corresponding member of the second, then the limit of the first sequence is at least the limit of the second:

- Limit-Order Lemma: ${ }^{31}$ If $(S, \mathcal{A}, \succsim)$ satisfies order and continuity, then for each pair of convergent sequences $\left(A_{i}\right),\left(B_{i}\right) \in \mathcal{A}^{\mathbb{N}}$ such that for each $j \in \mathbb{N}, A_{j} \succsim B_{j}$, we have $\lim \left(A_{i}\right) \succsim \lim \left(B_{i}\right)$.

Proof: Let $\left(A_{i}\right),\left(B_{i}\right) \in \mathcal{A}^{\mathbb{N}}$ satisfy the hypothesis. Since $\succsim$ is complete, by a standard argument ${ }^{32}$ there is $M \subseteq \mathbb{N}$ such that that $\left.\left(B_{i}^{\prime}\right) \equiv\left(B_{i}\right)\right|_{M}$ is a $\succsim$-monotonic sequence. Define $\left.\left(A_{i}^{\prime}\right) \equiv\left(A_{i}\right)\right|_{M}$. Necessarily $\lim \left(A_{i}\right)=\lim \left(A_{i}^{\prime}\right)$ and $\lim \left(B_{i}\right)=\lim \left(B_{i}^{\prime}\right)$.

CASE 1: $\left(B_{i}^{\prime}\right)$ is non-decreasing. Then for each pair $j, k \in \mathbb{N}$ with $k \geq j$,

$$
\begin{aligned}
A_{k}^{\prime} & \succsim B_{k}^{\prime} \\
& \succsim B_{j}^{\prime},
\end{aligned}
$$

so by continuity $\lim \left(A_{i}^{\prime}\right) \succsim B_{j}^{\prime}$. Thus by continuity, $\lim \left(A_{i}^{\prime}\right) \succsim \lim \left(B_{i}^{\prime}\right)$, so $\lim \left(A_{i}\right) \succsim$ $\lim \left(B_{i}\right)$.

\footnotetext{
${ }^{31}$ Variants of this result appear in Villegas (1964) and Arrow (1970). This particular result does not require $\mathcal{A}$ to be a $\sigma$-algebra; any Hausdorff space will do, as can be seen from the proof.

${ }^{32} \mathrm{~A}$ common proof of the Bolzano-Weierstrass Theorem includes a lemma stating that each real sequence has a monotonic subsequence; the standard proof of that lemma suffices here.
} 
CASE 2: $\left(B_{i}^{\prime}\right)$ is non-increasing. Then for each pair $j, k \in \mathbb{N}$ with $k \geq j$,

$$
\begin{aligned}
A_{j}^{\prime} & \succsim B_{j}^{\prime} \\
& \succsim B_{k}^{\prime},
\end{aligned}
$$

so by continuity $A_{j}^{\prime} \succsim \lim \left(B_{i}^{\prime}\right)$. Thus by continuity, $\lim \left(A_{i}^{\prime}\right) \succsim \lim \left(B_{i}^{\prime}\right)$, so $\lim \left(A_{i}\right) \succsim$ $\lim \left(B_{i}\right)$.

\section{Appendix 2}

In this appendix, we prove the Supercabinet Blueprint Lemma.

- Supercabinet Blueprint Lemma: If $(S, \mathcal{A}, \succsim)$ is a qualitative probability space that satisfies (monotone) continuity and has a supercabinet, then it has a unique representation $\mu \in \mathbb{M}^{\sigma}(\mathcal{A})$.

ProOF: Let $\left\{\mathcal{Z}_{v}\right\}_{v \in \mathcal{I}} \subseteq[\mathcal{A} / \sim]$ be a supercabinet.

○ Step 1: For each $k \in\{0,1, \ldots\}$, there is $Z \in \mathcal{Z}_{\left(\frac{1}{2}\right)^{k}}$ such that $Z \succ \emptyset$.

We proceed by induction on $k$. By [SC1], $S \in \mathcal{Z}_{1}$, and by nondegeneracy and monotonicity, $S \succ \emptyset$.

For the inductive hypothesis, assume $k \in\{0,1, \ldots\}$ is such that there is $Z \in \mathcal{Z}_{\left(\frac{1}{2}\right)^{k}}$ such that $Z \succ \emptyset$. By [SC2], there are disjoint $Z^{\prime}, Z^{\prime \prime} \in \mathcal{Z}_{\left(\frac{1}{2}\right)^{k+1}}$ such that $Z^{\prime} \cup Z^{\prime \prime} \sim Z$. By separability, $Z^{\prime} \succ \emptyset$, else $\emptyset \succsim Z^{\prime} \cup Z^{\prime \prime} \sim Z$, contradicting $Z \succ \emptyset$. By induction we are done.

- STEP 2: For each $v \in \mathcal{Z}$ such that $v>0$, there is $Z \in \mathcal{Z}_{v}$ such that $Z \succ \emptyset$.

Let $v \in \mathcal{Z}$ such that $v>0$. Since $\mathcal{2}$ is dense in $[0,1]$ there is $k \in \mathbb{Z}$ such that $v>\left(\frac{1}{2}\right)^{k}$. Since $v-\left(\frac{1}{2}\right)^{k} \in \mathcal{Z}$, by [SC2] there are disjoint $Z \in \mathcal{Z}_{\left(\frac{1}{2}\right)^{k}}$ and $Z^{\prime} \in \mathcal{Z}_{v-\left(\frac{1}{2}\right)^{k}}$ such that $Z \cup Z^{\prime} \in \mathcal{Z}_{v}$. By Step $1, Z \succ \emptyset$, and by monotonicity, $Z^{\prime} \succsim \emptyset$, so by separability, $Z \cup Z^{\prime} \succ \emptyset$.

○ Step 3: For each pair $v, v^{*} \in \mathcal{Z}$ such that $v^{*}>v$, each $A \in \mathcal{Z}_{v^{*}}$, and each $B \in \mathcal{Z}_{v}$, $A \succ B$.

Let $v, v^{*} \in \mathcal{L}$ such that $v^{*}>v$, let $A \in \mathcal{Z}_{v^{*}}$, and let $B \in \mathcal{Z}_{v}$. Since $v^{*}-v \in \mathcal{L}$, by [SC2] there are disjoint $Z \in \mathcal{Z}_{v}$ and $Z^{\prime} \in \mathcal{Z}_{v^{*}-v}$ such that $Z \cup Z^{\prime} \in \mathcal{Z}_{v^{*}}$. Since $v^{*}-v>0$ (and since $\left.\mathcal{Z}_{v^{*}-v} \in[\mathcal{A} / \sim]\right)$, by Step $2, Z^{\prime} \succ \emptyset$. Thus by separability, $A \sim Z \cup Z^{\prime} \succ Z \sim B$.

○ Step 4: Define $\mu: \mathcal{A} \rightarrow[0,1]$.

For each $v \in \mathbb{Z}$ and each $A \in \mathcal{Z}_{v}$, define $\mu(A) \equiv v$.

Let $A \in \mathcal{A} \backslash \cup_{v \in \mathbb{Z}} \mathcal{Z}_{v}$. Define

$$
\begin{aligned}
& \mathcal{D}^{-} \equiv\left\{v \in \mathcal{L} \mid B \in Z_{v} \text { implies } A \succsim B\right\}, \text { and } \\
& \mathcal{2}^{+} \equiv\left\{v \in \mathcal{L} \mid B \in Z_{v} \text { implies } B \succsim A\right\} .
\end{aligned}
$$


By monotonicity, $S \succsim A \succsim \emptyset$, so by [SC1], $0 \in \mathbb{2}^{-}$and $1 \in \mathbb{2}^{+}$. By Step 3, for each pair $v, v^{*} \in \mathbb{2}$ such that $v^{*}>v$, (1) $v^{*} \in \mathbb{2}^{-}$implies $v \in \mathbb{2}^{-} \backslash \mathcal{L}^{+}$, and (2) $v \in \mathcal{L}^{+}$implies $v^{*} \in \mathbb{2}^{+} \backslash \mathbb{2}^{-}$. Thus $\inf \left(\mathscr{Q}^{+}\right), \sup \left(\mathcal{L}^{-}\right)$are defined, and moreover $\inf \left(\mathbb{2}^{+}\right) \geq \sup \left(\mathcal{L}^{-}\right)$. Since $\mathbb{2}^{-} \cup \mathbb{2}^{+}=\mathbb{2}$ and $\mathcal{L}$ is dense in $[0,1], \inf \left(\mathscr{Q}^{+}\right)=\sup \left(\mathbb{2}^{-}\right)$. Define

$$
\begin{aligned}
\mu(A) & \equiv \inf \left(\mathscr{Q}^{+}\right) \\
& =\sup \left(\mathcal{L}^{-}\right) .
\end{aligned}
$$

○ SteP 5: For each pair $A, B \in \mathcal{A}, \mu(A)>\mu(B)$ implies $A \succ B$.

Let $A, B \in \mathcal{A}$ such that $\mu(A)>\mu(B)$. Since $\mathbb{P}$ is dense in $[0,1]$, there are $v^{\prime}, v^{\prime \prime} \in \mathbb{Z}$ such that $\mu(A)>v^{\prime \prime}>v^{\prime}>\mu(B)$. Let $Z_{v^{\prime}} \in \mathcal{Z}_{v^{\prime}}$ and $Z_{v^{\prime \prime}} \in \mathcal{Z}_{v^{\prime \prime}}$. By Step 3, $Z_{v^{\prime \prime}} \succ Z_{v^{\prime}}$.

If $A \in \cup \mathcal{Z}_{v}$, then by construction, $A \in \mathcal{Z}_{\mu(A)}$, so by Step $3, A \succ Z_{v^{\prime \prime}}$. If $A \notin \cup \mathcal{Z}_{v}$, then by construction, $\inf \left\{v \in \mathbb{2} \mid C \in \mathcal{Z}_{v}\right.$ implies $\left.C \succsim A\right\}>v^{\prime \prime}$, so $A \succ Z_{v^{\prime \prime}}$.

If $B \in \cup \mathcal{Z}_{v}$, then by construction, $B \in \mathcal{Z}_{\mu(B)}$, so by Step $3, Z_{v^{\prime}} \succ B$. If $B \notin \cup \mathcal{Z}_{v}$, then by construction, $v^{\prime}>\sup \left\{v \in \mathcal{L} \mid C \in \mathcal{Z}_{v}\right.$ implies $\left.B \succsim C\right\}$, so $Z_{v^{\prime}} \succ B$.

Thus $A \succ Z_{v^{\prime \prime}} \succ Z_{v^{\prime}} \succ B$.

○ Step 6: For each pair $A, B \in \mathcal{A}, \mu(A)=\mu(B)$ implies $A \sim B$.

Let $A, B \in \mathcal{A}$ such that $\mu(A)=\mu(B)$. We proceed with three cases whose proofs are similar.

CASE 1: $\mu(A)=0$. Since $\mathcal{2}$ is dense in $[0,1]$, there is decreasing $\left(v_{i}^{+}\right) \in \mathcal{2}^{\mathbb{N}}$ such that $\lim v_{i}^{+}=0$. For each $i \in \mathbb{N}$, define $v_{i}^{-} \equiv 0$. Since $\lim v_{i}^{+}=\lim v_{i}^{-}$, by [SC3] there are convergent $\left(A_{i}^{+}\right),\left(A_{i}^{-}\right) \in \mathcal{A}^{\mathbb{N}}$ such that

(i) for each $i \in \mathbb{N}, A_{i}^{+} \in \mathcal{Z}_{v_{i}^{+}}$and $A_{i}^{-} \in \mathcal{Z}_{v_{i}^{-}}$, and

(ii) $\lim A_{i}^{+}=\lim A_{i}^{-}$.

By construction, for each $i \in \mathbb{N}, \mu\left(A_{i}^{+}\right)>0=\mu(A)$, so by Step $5, A_{i}^{+} \succ A$. Thus by continuity, $\lim A_{i}^{+} \succsim A$. By [SC1], for each $i \in \mathbb{N}, A_{i}^{-} \sim \emptyset$, so by continuity, $\lim A_{i}^{-} \sim \emptyset$. Altogether, $\emptyset \succsim A$, so by monotonicity, $A \sim \emptyset$. By a symmetric argument, $B \sim \emptyset$, so $A \sim B$.

CASE 2: $\mu(A)=1$. Since $\mathbb{2}$ is dense in $[0,1]$, there is increasing $\left(v_{i}^{-}\right) \in \mathscr{2}^{\mathbb{N}}$ such that $\lim v_{i}^{-}=1$. For each $i \in \mathbb{N}$, define $v_{i}^{+} \equiv 1$. Since $\lim v_{i}^{+}=\lim v_{i}^{-}$, by [SC3] there are convergent $\left(A_{i}^{+}\right),\left(A_{i}^{-}\right) \in \mathcal{A}^{\mathbb{N}}$ such that

(i) for each $i \in \mathbb{N}, A_{i}^{+} \in \mathcal{Z}_{v_{i}^{+}}$and $A_{i}^{-} \in \mathcal{Z}_{v_{i}^{-}}$, and

(ii) $\lim A_{i}^{+}=\lim A_{i}^{-}$.

By construction, for each $i \in \mathbb{N}, \mu(A)=1>\mu\left(A_{i}^{-}\right)$, so by Step $5, A \succ A_{i}^{-}$. Thus by continuity, $A \succsim \lim A_{i}^{-}$. By [SC1], for each $i \in \mathbb{N}, A_{i}^{+} \sim S$, so by continuity, $\lim A_{i}^{+} \sim S$. Altogether, $A \succsim S$, so by monotonicity, $A \sim S$. By a symmetric argument, $B \sim S$, so $A \sim B$.

CASE 3: $\mu(A) \in(0,1)$. Since $\mathcal{2}$ is dense in $[0,1]$, there are decreasing $\left(v_{i}^{+}\right) \in \mathbb{2}^{\mathbb{N}}$ such that $\lim v_{i}^{+}=\mu(A)$ and increasing $\left(v_{i}^{-}\right) \in \mathcal{2}^{\mathbb{N}}$ such that $\lim v_{i}^{-}=\mu(A)$. Since $\lim v_{i}^{+}=\lim v_{i}^{-}$, by [SC3] there are convergent $\left(A_{i}^{+}\right),\left(A_{i}^{-}\right) \in \mathcal{A}^{\mathbb{N}}$ such that

(i) for each $i \in \mathbb{N}, A_{i}^{+} \in \mathcal{Z}_{v_{i}^{+}}$and $A_{i}^{-} \in \mathcal{Z}_{v_{i}^{-}}$, and 
(ii) $\lim A_{i}^{+}=\lim A_{i}^{-}$.

By construction, for each $i \in \mathbb{N}, \mu\left(A_{i}^{+}\right)>\mu(A)>\mu\left(A_{i}^{-}\right)$, so by Step $5, A_{i}^{+} \succ A \succ A_{i}^{-}$. Thus by continuity, $\lim A_{i}^{+} \succsim A \succsim \lim A_{i}^{-}=\lim A_{i}^{+}$, so $A \sim \lim A_{i}^{+}$. By a symmetric argument, $B \sim \lim A_{i}^{+}$, so $A \sim B$.

○ $\operatorname{STEP} 7: \mu$ represents $\succsim$.

Immediate from Step 5 and Step 6.

○ STEP 8: For each disjoint pair $A, B \in \mathcal{A}, \mu(A)+\mu(B) \leq 1$.

Let $A, B \in \mathcal{A}$ be disjoint. Assume, by way of contradiction, $\mu(A)+\mu(B)>1$. Since $\mathcal{2}$ is dense in $[0,1]$, there are $v, v^{\prime} \in \mathcal{Z}$ such that $\mu(A)>v, \mu(B)>v^{\prime}$, and $v+v^{\prime}>1$. Then $v>1-v^{\prime}$. By [SC2], there are disjoint $Z_{v^{\prime}} \in \mathcal{Z}_{v^{\prime}}$ and $Z_{1-v^{\prime}} \in \mathcal{Z}_{1-v^{\prime}}$ such that $Z_{v^{\prime}} \cup Z_{1-v^{\prime}} \in \mathcal{Z}_{1}$. Then $Z_{v^{\prime}} \cup Z_{1-v^{\prime}} \sim S$. Since $\mu(A)>v>1-v^{\prime}=\mu\left(Z_{1-v^{\prime}}\right)$ and $\mu(B)>$ $v^{\prime}=\mu\left(Z_{v^{\prime}}\right)$, by Step 7, $A \succ Z_{1-v^{\prime}}$ and $B \succ Z_{v^{\prime}}$. But then by the Domination Lemma, $A \cup B \succ Z_{1-v^{\prime}} \cup Z_{v^{\prime}} \sim S$, contradicting monotonicity.

○ STEP 9: For each disjoint pair $A, B \in \cup \mathcal{Z}_{v}, \mu(A \cup B)=\mu(A)+\mu(B)$.

Let $A, B \in \cup \mathcal{Z}_{v}$ be disjoint. By construction, $A \in \mathcal{Z}_{\mu(A)}$ and $B \in \mathcal{Z}_{\mu(B)}$, and by Step 8, $\mu(A)+\mu(B) \leq 1$, so by [SC2] and the Domination Lemma, $A \cup B \in \mathcal{Z}_{\mu(A)+\mu(B)}$. Thus by construction, $\mu(A \cup B)=\mu(A)+\mu(B)$.

- SteP 10: For each disjoint pair $A, B \in \mathcal{A}, \mu(A \cup B)=\mu(A)+\mu(B)$.

Let $A, B \in \mathcal{A}$ be disjoint. By Step $8, \mu(A)+\mu(B) \in[0,1]$. We begin by proving that $1>\mu(A)+\mu(B)$ implies $\mu(A)+\mu(B) \geq \mu(A \cup B)$. In this case, since $\mathcal{2}$ is dense in $[0,1]$, there are non-increasing $\left(v_{i}\right),\left(w_{i}\right) \in \mathcal{2}^{\mathbb{N}}$ such that

(1) for each $i \in \mathbb{N}, v_{i} \geq \mu(A), w_{i} \geq \mu(B)$, and $1 \geq v_{i}+w_{i}$; and

(2) $\lim v_{i}=\mu(A)$ and $\lim w_{i}=\mu(B)$.

By [SC4], there are convergent $\left(A_{i}\right),\left(B_{i}\right) \in \mathcal{A}^{\mathbb{N}}$ such that

(1) for each $i \in \mathbb{N}, A_{i} \in \mathcal{Z}_{v_{i}}$ and $B_{i} \in \mathcal{Z}_{w_{i}}$; and

(2) for each $i \in \mathbb{N}, A_{i} \cap B_{i}=\emptyset$.

Define $A_{\infty} \equiv \lim A_{i}$ and $B_{\infty} \equiv \lim B_{i}$. By definition of $\mu$, for each $i \in \mathbb{N}, \mu\left(A_{i}\right)=v_{i}$ and $\mu\left(B_{i}\right)=w_{i}$.

Let $\epsilon>0$. Since $\lim v_{i}=\mu(A)$ and $\lim w_{i}=\mu(B)$, there is $i^{*} \in \mathbb{N}$ such that $i \geq i^{*}$ implies $\mu(A)+\frac{\epsilon}{2}>v_{i}$ and $\mu(B)+\frac{\epsilon}{2}>w_{i}$. Then $i \geq i^{*}$ implies $\mu(A)+\mu(B)+\epsilon>v_{i}+w_{i}=$ $\mu\left(A_{i}\right)+\mu\left(B_{i}\right)$, so by Step $9, i \geq i^{*}$ implies $\mu(A)+\mu(B)+\epsilon>\mu\left(A_{i} \cup B_{i}\right)$.

By Step $7, A_{1} \succsim A_{2} \succsim \ldots$ and $B_{1} \succsim B_{2} \succsim \ldots$. Then for each $i \in \mathbb{N}$, since $A_{\infty}$ is the limit of the subsequence $\left(A_{j}\right)_{j \geq i}$, thus by continuity $A_{i} \succsim A_{\infty}$, and similarly $B_{i} \succsim B_{\infty}$. Moreover, for each $i \in \mathbb{N}, A_{i} \cap B_{i}=\emptyset$; thus by the Domination Lemma, $A_{i} \cup B_{i} \succsim A_{\infty} \cup B_{\infty}$, so by Step $7, \mu\left(A_{i} \cup B_{i}\right) \geq \mu\left(A_{\infty} \cup B_{\infty}\right)$.

Altogether, for each $\epsilon>0$, there is $i \in \mathbb{N}$ such that $\mu(A)+\mu(B)+\epsilon>\mu\left(A_{i} \cup B_{i}\right) \geq$ $\mu\left(A_{\infty} \cup B_{\infty}\right)$; thus $\mu(A)+\mu(B) \geq \mu\left(A_{\infty} \cup B_{\infty}\right)$.

By Step 7, for each $i \in \mathbb{N}, A_{i} \succsim A$ and $B_{i} \succsim B$, so by continuity, $A_{\infty} \succsim A$ and $B_{\infty} \succsim B$. Moreover, since for each $i \in \mathbb{N}, A_{i} \cap B_{i}=\emptyset$, thus $A_{\infty} \cap B_{\infty}=\emptyset$, so by 
the Domination Lemma, $A_{\infty} \cup B_{\infty} \succsim A \cup B$. By Step 7 and the previous observation, $\mu(A)+\mu(B) \geq \mu\left(A_{\infty} \cup B_{\infty}\right) \geq \mu(A \cup B)$, as desired.

The argument that $\mu(A)+\mu(B)>0$ implies $\mu(A \cup B) \geq \mu(A)+\mu(B)$ is almost completely symmetric, except that applications of the Domination Lemma involve establishing that the 'other' pair is disjoint, which is straightforward. Altogether, then, $\mu(A \cup B)=\mu(A)+\mu(B)$ whether $\mu(A)+\mu(B)$ is 0,1 , or in between.

○ STEP 11: $\mu \in \mathbb{M}^{\sigma}(\mathcal{A})$.

Since $\mu(S)=1$, by Step 10 and induction, $\mu \in \mathbb{M}(\mathcal{A})$. By Step 7, $\mu$ represents $\succsim$, so by Theorem V1, $\mu \in \mathbb{M}^{\sigma}(\mathcal{A})$.

○ STEP 12: If $\mu^{\prime} \in \mathbb{M}^{\sigma}(\mathcal{A})$ represents $\succsim$, then $\mu^{\prime}=\mu$.

If $\mu^{\prime} \in \mathbb{M}^{\sigma}(\mathcal{A})$ represents $\succsim$, then by [SC1] and [SC2], it is immediate that $\mu^{\prime}$ must be defined as in Step 4.

\section{Appendix 3}

In this appendix, we prove our primary lemmas about greedy transforms: the Idempotence Lemma, the 1-AS Lemma, the Greedy Removal Lemma, and the Convergence Lemma. We abuse language in our informal summaries of these lemmas, writing that an event satisfies $k$-AS instead of writing that its associated subspace does.

The Idempotence Lemma states that each greedy transform is idempotent, and for convenience includes the easy corollary that two images of a greedy transform that are equally likely are in fact equivalent.

- Idempotence Lemma: If $(S, \mathcal{A}, \succsim)$ is a purely- $\sigma$-catalogued qualitative probability space satisfying (monotone) continuity, then for each pair $A, B \in \mathcal{A}, \mathcal{G}^{A}\left(\mathcal{G}^{A}(B)\right)=$ $\mathcal{G}^{A}(B)$. Moreover, for each $A \in \mathcal{A}$ and each pair $B, B^{\prime} \in \mathcal{A}, \mathcal{G}^{A}(B) \sim \mathcal{G}^{A}\left(B^{\prime}\right)$ implies $\mathcal{G}^{A}(B)=\mathcal{G}^{A}\left(B^{\prime}\right)$

Proof: We proceed by induction on $s$, covering the base case with our inductive hypothesis. Assume $s \in S$ is such that for each $s^{\prime} \in S$,

$$
\mathcal{G}^{A}\left(\mathcal{G}^{A}(B)\right) \cap\left\{s^{\prime} \in S \mid s^{\prime}<s\right\}=\mathcal{G}^{A}(B) \cap\left\{s^{\prime} \in S \mid s^{\prime}<s\right\} .
$$

If $s \in \mathcal{G}^{A}(B)$, then by construction,

$$
\begin{aligned}
\mathcal{G}^{A}(B) & \succsim\left[\mathcal{G}^{A}(B) \cap\left\{s^{\prime} \in S \mid s^{\prime}<s\right\}\right] \cup\{s\} \\
& =\left[\mathcal{G}^{A}\left(\mathcal{G}^{A}(B)\right) \cap\left\{s^{\prime} \in S \mid s^{\prime}<s\right\}\right] \cup\{s\},
\end{aligned}
$$

so by construction, $s \in \mathcal{G}^{A}\left(\mathcal{G}^{A}(B)\right)$.

If $s \notin \mathcal{G}^{A}(B)$, then by construction,

$$
\begin{aligned}
{\left[\mathcal{G}^{A}\left(\mathcal{G}^{A}(B)\right) \cap\left\{s^{\prime} \in S \mid s^{\prime}<s\right\}\right] \cup\{s\} } & =\left[\mathcal{G}^{A}(B) \cap\left\{s^{\prime} \in S \mid s^{\prime}<s\right\}\right] \cup\{s\} \\
& \succ B \\
& \succsim \mathcal{G}^{A}(B),
\end{aligned}
$$


so by construction, $s \notin \mathcal{G}^{A}\left(\mathcal{G}^{A}(B)\right)$. Thus

$$
\mathcal{G}^{A}\left(\mathcal{G}^{A}(B)\right) \cap\left\{s^{\prime} \in S \mid s^{\prime}<s+1\right\}=\mathcal{G}^{A}(B) \cap\left\{s^{\prime} \in S \mid s^{\prime}<s+1\right\} .
$$

By induction, $\mathcal{G}^{A}\left(\mathcal{G}^{A}(B)\right)=\mathcal{G}^{A}(B)$.

Now assume $A \in \mathcal{A}$ and $B, B^{\prime} \in \mathcal{A}$ are such that $\mathcal{G}^{A}(B) \sim \mathcal{G}^{A}\left(B^{\prime}\right)$. Then $\mathcal{G}^{A}(B)=$ $\mathcal{G}^{A}\left(\mathcal{G}^{A}(B)\right)=\mathcal{G}^{A}\left(\mathcal{G}^{A}\left(B^{\prime}\right)\right)=\mathcal{G}^{A}\left(B^{\prime}\right)$, as desired.

The 1-AS Lemma is the ordinal analogue of Kakeya's observation (Kakeya, 1914; Kakeya, 1915): 1- $A S$ of $A$ guarantees that applying $A$ 's greedy transform to an event $B$ that is no larger than $A$ yields an event as likely as $B$.

- 1-AS Lemma: If $(S, \mathcal{A}, \succsim)$ is a purely- $\sigma$-catalogued qualitative probability space satisfying (monotone) continuity, then for each $A \in \mathcal{A}$ such that $\left.\succsim\right|_{A}$ satisfies $1-A S$ and each $B \in \mathcal{A}$ such that $A \succsim B, \mathcal{G}^{A}(B) \sim B$.

Proof: If $A=\emptyset$ the result is trivial, so assume $A \neq \emptyset$. Then by $1-A S,|A|=|\mathbb{N}|$. For convenience, re-index $S$ so that (1) $A=\mathbb{N}$, (2) index order is preserved for $A$, and (3) members of $S \backslash A$ are not indexed by natural numbers. Since $B \succsim \mathcal{G}^{A}(B)$, it suffices to show $\mathcal{G}^{A}(B) \succsim B$.

CAse 1: $\left|A \backslash \mathcal{G}^{A}(B)\right|=0$. Then $\mathcal{G}^{A}(B)=A \succsim B$.

CASE 2: $0<\left|A \backslash \mathcal{G}^{A}(B)\right|<|\mathbb{N}|$. Define $s^{*} \equiv \max \left(A \backslash \mathcal{G}^{A}(B)\right)$. By construction, $\left[\mathcal{G}^{A}(B) \backslash\left\{s^{*}+1, s^{*}+2, \ldots\right\} \cup\left\{s^{*}\right\}\right] \succ B$. Since $\left.\succsim\right|_{A}$ satisfies $1-A S$, thus by separability

$$
\begin{aligned}
\mathcal{G}^{A}(B) & =\left[\mathcal{G}^{A}(B) \backslash\left\{s^{*}+1, s^{*}+2, \ldots\right\}\right] \cup\left\{s^{*}+1, s^{*}+2, \ldots\right\} \\
& \succsim\left[\mathcal{G}^{A}(B) \backslash\left\{s^{*}+1, s^{*}+2, \ldots\right\}\right] \cup\left\{s^{*}\right\} \\
& \succ B
\end{aligned}
$$

But this contradicts $B \succsim \mathcal{G}^{A}(B)$, so in fact Case 2 is impossible.

CASE 3: $\left|A \backslash \mathcal{G}^{A}(B)\right|=|\mathbb{N}|$. By construction, for each $s \in A \backslash \mathcal{G}^{A}(B),\left[\mathcal{G}^{A}(B) \backslash\{s+\right.$ $1, s+2, \ldots\}] \cup\{s\} \succ B$. Thus by continuity,

$$
\begin{aligned}
\mathcal{G}^{A}(B) & =\lim _{s \in S^{\prime} \backslash \mathcal{G}^{A}(B)}\left[\mathcal{G}^{A}(B) \backslash\{s+1, s+2, \ldots\}\right] \cup\{s\} \\
& \succsim B .
\end{aligned}
$$

The Greedy Removal Lemma is the ordinal analogue of Komornik's observation (Komornik, 2015): if $A$ is $(k+1)-A S$, then removing an image of its greedy transform yields a subevent that is $k-A S$.

- Greedy Removal Lemma: If $(S, \mathcal{A}, \succsim)$ is a purely- $\sigma$-catalogued qualitative probability space satisfying (monotone) continuity, then for each $k \in \mathbb{N}$, each $A \in \mathcal{A}$ such that $\succsim_{A}$ satisfies $(k+1)-A S$, and each $B \in \mathcal{A}$,

$$
\left.\succsim\right|_{A \backslash \mathcal{G}^{A}(B)} \text { satisfies } k \text {-AS. }
$$


Proof: Let $k \in \mathbb{N}$ and assume $\left.\succsim\right|_{A}$ satisfies $k$-AS. If $A=\emptyset$ or $B \succsim A$, the result is trivial, so assume $A \succ B \succsim \emptyset$. By $1-A S,|A|=|\mathbb{N}|$. For convenience, re-index $S$ so that (i) $A=\mathbb{N}$, (2) index order is preserved for $A$, and (iii) members of $S \backslash A$ are not indexed by natural numbers.

○ STEP 1: If $k^{\prime}<k+1$ greedy transform sets $G_{i}$ are iteratively removed from $A$, and if $A \backslash \cup G_{i}$ is nonempty, then $\left.\succsim\right|_{A \backslash \cup G_{i}}$ satisfies $1-A S$.

Let $k^{\prime} \in \mathbb{N}$ such that $k^{\prime}<k+1$, and let $B_{1}, \ldots, B_{k^{\prime}} \in \mathcal{A}$. Define $A_{1} \equiv A$, and for each $i \in\left\{1,2, \ldots, k^{\prime}\right\}$, define:

- $G_{i} \equiv \mathcal{G}^{A_{i}}\left(B_{i}\right)$,

- $A_{i+1} \equiv A_{i} \backslash G_{i}$.

By construction, the $G_{i}$ are pairwise disjoint. We claim $A \backslash \cup G_{i} \neq \emptyset$ implies $\left.\succsim\right|_{A \backslash \cup G_{i}}$ satisfies 1 - $A S$. Indeed, let $s \in A \backslash \cup G_{i}$. Since $\left.\succsim\right|_{A}$ satisfies $(k+1)-A S$, there are pairwise disjoint $B_{1}, \ldots, B_{k+1} \subseteq\{s+1, s+2, \ldots\}$ such that for each $i \in\{1,2, \ldots, k+1\}, B_{i} \succsim\{s\}$. By construction, for each $i \in\left\{1,2, \ldots, k^{\prime}\right\},\{s\} \succsim G_{i} \cap\{s+1, s+2, \ldots\}$. Thus for each $i \in\left\{1,2, \ldots, k^{\prime}\right\}, B_{i} \succsim G_{i} \cap\{s+1, s+2, \ldots\}$. By repeated application of the Domination Lemma,

$$
\begin{aligned}
B_{1} & \succsim G_{1} \cap\{s+1, s+2, \ldots\}, \\
B_{1} \cup B_{2} & \succsim\left(G_{1} \cap\{s+1, s+2, \ldots\}\right) \cup\left(G_{2} \cap\{s+1, s+2, \ldots\}\right), \\
\vdots & \\
\bigcup_{i=1}^{k^{\prime}} B_{i} & \succsim \bigcup_{i=1}^{k^{\prime}}\left(G_{i} \cap\{s+1, s+2, \ldots\}\right)
\end{aligned}
$$

Thus if $\{s\} \succ\left[\{s+1, s+2, \ldots\} \backslash \cup G_{i}\right]$, then $B_{k^{\prime}+1} \succ\left[\{s+1, s+2, \ldots\} \backslash \cup G_{i}\right]$, so by the Domination Lemma,

$$
\begin{aligned}
\bigcup_{i=1}^{k^{\prime}+1} B_{i} & \succ \bigcup_{i=1}^{k^{\prime}}\left(G_{i} \cap[A \cap\{s+1, s+2, \ldots\}]\right) \cup\left[\{s+1, s+2, \ldots\} \backslash \cup G_{i}\right] \\
& =\{s+1, s+2, \ldots\}
\end{aligned}
$$

contradicting monotonicity. Thus $\left[\{s+1, s+2, \ldots\} \backslash \cup G_{i}\right] \succsim\{s\}$. Since $s \in A \backslash G_{i}$ was arbitrary, thus $\left.\succsim\right|_{A \backslash \cup G_{i}}$ satisfies $1-A S$.

○ Step 2: Conclude.

Let $B \in \mathcal{A}$ such that $A \succ B$ and let $G_{1}=\mathcal{G}^{A}(B)$. Then there is $s \in A \backslash G_{1}$, else by monotonicity $B \succsim G_{1}=A \succ B$, contradicting $B \sim B$. By Step 1, $\left.\succsim\right|_{A \backslash G_{1}}$ satisfies 1-AS.

Let $s \in A \backslash G_{1}$ and define $A_{1} \equiv\{s+1, s+2, \ldots\}$. Then $\left.\succsim\right|_{A_{1}}$ satisfies $(k+1)-A S$ and $\left.\succsim\right|_{A_{1} \backslash G_{1}}$ satisfies $1-A S$. Thus there are pairwise disjoint $B_{1}, \ldots, B_{k+1} \subseteq A_{1}$ such that for each $i \in\{1,2, \ldots, k+1\}, B_{i} \succsim\{s\}$.

Define $A_{2} \equiv A_{1} \backslash G_{1}$, and for each $i \in\{2,3, \ldots, k+1\}$, define:

- $G_{i} \equiv \mathcal{G} \leq\left(A_{i},\{s\}\right)$, 
- $A_{i+1} \equiv A_{i} \backslash G_{i}$.

By construction, the $G_{i}$ are pairwise disjoint. Assume, by way of contradiction, there is $i \in\{2,3, \ldots, k+1\}$ such that $\{s\} \succ A_{i}$. Let $i^{*}$ be the least such $i$. Then $B_{i^{*}} \succsim\{s\} \succ A_{i^{*}}$, and for each $i \in\left\{1, \ldots, i^{*}-1\right\}, B_{i} \succsim\{s\} \succsim G_{i}$. But then by repeated application of the Domination Lemma as in Step 1,

$$
\begin{aligned}
\bigcup_{i=1}^{i^{*}-1} B_{i} & \succ \bigcup_{i=1}^{i^{*}-1} G_{i} \cup A_{i^{*}} \\
& =A_{1}
\end{aligned}
$$

contradicting monotonicity.

Thus for each $i \in\{2,3, \ldots, k+1\}, A_{i} \succsim\{s\}$, so by monotonicity $A_{i}$ is nonempty, so by Step $\left.1 \succsim\right|_{A_{i}}$ satisfies $1-A S$, so by the $1-A S$ Lemma $B_{i} \sim\{s\}$. Since $s \in A \backslash G_{1}$ was arbitrary, $\left.\succsim\right|_{A \backslash G_{1}}$ satisfies $k$ - $A S$.

The Convergence Lemma concerns generalized greedy transforms, each of which takes as input a list of events and outputs the same number of events:

Definition: For each $A \in \mathcal{A}$ and each $k \in \mathbb{N}$, the greedy transform $\mathcal{G}^{A}: \mathcal{A}^{k} \rightarrow \mathcal{A}^{k}$ is defined as follows. For each $\left(B_{1}, B_{2}, \ldots, B_{k}\right) \in \mathcal{A}^{k}, \mathcal{G}^{A}\left(B_{1}, B_{2}, \ldots, B_{k}\right) \in \mathcal{A}^{k}$ is defined recursively by:

- $\mathcal{G}_{0}^{A}\left(B_{1}, B_{2}, \ldots, B_{k}\right) \equiv \emptyset$, and

- for each $i \in\{1,2, \ldots, k\}$,

$$
\mathcal{G}_{i}^{A}\left(B_{1}, B_{2}, \ldots, B_{k}\right) \equiv \mathcal{G}^{S \backslash\left(\cup_{j<i} \mathcal{G}_{j}^{A}\left(B_{1}, B_{2}, \ldots, B_{k}\right)\right.}\left(A_{i}\right) .
$$

The lemma states that for nice sequences of event lists, the associated sequence of event lists output by a generalized greedy transform converges.

- Convergence Lemma: If $(S, \mathcal{A}, \succsim)$ is a purely- $\sigma$-catalogued qualitative probability space satisfying (monotone) continuity, $k \in \mathbb{N}$, and $\left(B_{i}^{1}\right),\left(B_{i}^{2}\right), \ldots,\left(B_{i}^{k}\right) \in \mathcal{A}^{\mathbb{N}}$ are such that

(1) for each $j \in\{1,2, \ldots, k\},\left(B_{i}^{j}\right)$ is monotonic, and

(2) for each $i \in \mathbb{N}, B_{i}^{1}, B_{i}^{2}, \ldots, B_{i}^{k}$ are pairwise disjoint, then for each $A \in \mathcal{A}$ and each $j \in\{1,2, \ldots, k\},\left(\mathcal{G}_{j}^{A}\left(B_{i}^{1}, B_{i}^{2}, \ldots, B_{i}^{k}\right)\right) \in \mathcal{A}^{\mathbb{N}}$ is convergent.

Proof: Let $A \in \mathcal{A}$, let $k \in \mathbb{N}$, and let $\left(B_{i}^{1}\right),\left(B_{i}^{2}\right), \ldots,\left(B_{i}^{k}\right) \in \mathcal{A}^{\mathbb{N}}$ satisfy the hypotheses. For each $i \in \mathbb{N}$ and each $j \in\{1,2, \ldots, k\}$, define $G_{i}^{j} \in \mathcal{A}$ by:

$$
G_{i}^{j} \equiv \mathcal{G}_{j}^{A}\left(B_{i}^{1}, B_{i}^{2}, \ldots, B_{i}^{k}\right) .
$$

To prove $\left(G_{i}^{1}\right),\left(G_{i}^{2}\right), \ldots,\left(G_{i}^{k}\right)$ are convergent, we proceed by induction. We cover the base step with our inductive hypothesis on $j^{*}$ : assume $j^{*} \in\{1,2, \ldots, k-1\}$ is such that for each $j \in\{1,2, \ldots, k\}$ such that $j<j^{*},\left(G_{i}^{j}\right)$ is convergent. We claim $\left(G_{i}^{j^{*}}\right)$ is convergent.

Within the current inductive argument, we make a second inductive argument. We cover the base step with our inductive hypothesis on $s$ : assume $s \in S$ is such that for each $s^{\prime}<s$, there is $i^{*} \in \mathbb{N}$ such that for each $j \in\left\{1,2, \ldots, j^{*}\right\}$, either 


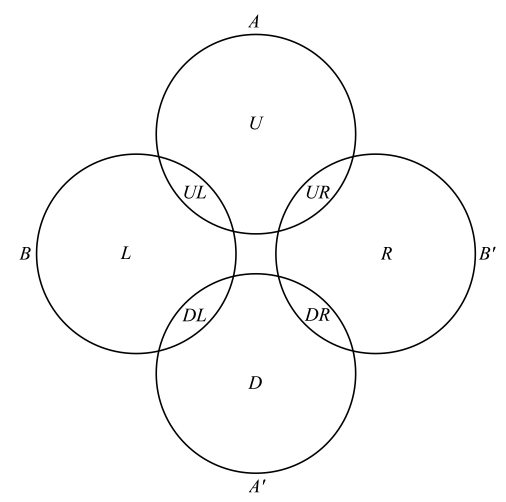

Figure 4: Euler diagram for $A, B, A^{\prime}, B^{\prime}$. For example, $U L \equiv A \cap B$ and $D \equiv A^{\prime} \backslash\left(B \cup B^{\prime}\right)$.

(1) $i \geq i^{*}$ implies $s^{\prime} \in G_{i}^{j}$, or

(2) $i \geq i^{*}$ implies $s^{\prime} \in G_{i}^{j}$.

By the inductive hypothesis on $j^{*}$, there is $i^{*} \in \mathbb{N}$ such that for each $j \in\{1,2, \ldots, k\}$ such that $j<j^{*}$, either

(1) $i \geq i^{*}$ implies $s \in G_{i}^{j}$, or

(2) $i \geq i^{*}$ implies $s \in G_{i}^{j}$.

Assume, by way of contradiction, that for each $i^{* *} \in \mathbb{N}$ there are $i_{1} \geq i^{* *}$ and $i_{2} \geq i^{* *}$ such that $s \in G_{i_{1}}^{j^{*}}$ and $s \notin G_{i_{2}}^{j^{*}+1}$. Then there are $i_{1}, i_{2}, i_{3} \in \mathbb{N}$ with $i_{3}>i_{2}>i_{1}>i^{*}$ such that $s \in G_{i_{1}}^{j^{*}}, s \notin G_{i_{2}}^{j^{*}}$, and $s \in G_{i_{3}}^{j^{*}}$. By definition of $i^{*}, G_{i_{1}}^{j^{*}} \cap\left\{s^{\prime} \in S \mid s^{\prime}<s\right\}=G_{i_{2}}^{j^{*}} \cap\left\{s^{\prime} \in\right.$ $\left.S \mid s^{\prime}<s\right\}=G_{i_{3}}^{j^{*}} \cap\left\{s^{\prime} \in S \mid s^{\prime}<s\right\}$. Thus by construction, $B_{i_{1}}^{j^{*}} \succsim G_{i_{1}}^{j^{*}} \cap\left\{s^{\prime} \in S \mid s^{\prime}<s\right\} \cup$ $\{s\} \succ B_{i_{2}}^{j^{*}}$, so $\left(B_{i}^{j^{*}}\right)$ is non-increasing. But then $B_{i_{3}}^{j^{*}} \succsim G_{i_{1}}^{j^{*}} \cap\left\{s^{\prime} \in S \mid s^{\prime}<s\right\} \cup\{s\} \succ B_{i_{2}}^{j^{*}}$, contradicting that $\left(B_{i}^{j^{*}}\right)$ is non-increasing.

By induction on $s,\left(G_{i}^{j^{*}}\right)$ is convergent. By induction on $j^{*},\left(G_{i}^{1}\right),\left(G_{i}^{2}\right), \ldots,\left(G_{i}^{k}\right)$ are convergent.

\section{Appendix 4}

In this appendix, we prove our primary lemmas about qualitative halves: the HalfEquivalence Lemma, the First Halving Lemma, and the Second Halving Lemma.

The Half-Equivalence Lemma states that for any two disjoint pairs whose unions are equally likely such that each pair's members are equally likely, all four events are equally likely.

- Half-Equivalence Lemma: If $(S, \mathcal{A}, \succsim)$ is a qualitative probability space, then for each disjoint pair $A, A^{\prime} \in \mathcal{A}$ and each disjoint pair $B, B^{\prime} \in \mathcal{A}$, if

(1) $A \sim A^{\prime}$

(2) $B \sim B^{\prime}$, and

(3) $A \cup A^{\prime} \sim B \cup B^{\prime}$,

then $A \sim B$.

Proof: For convenience, label the components of the Euler diagram for $A, A^{\prime}, B, B^{\prime}$ according to Figure 4:

Assume, by way of contradiction, $A \Varangle B$; without loss of generality, assume $A \succ B$. 
We claim $D L \cup D \succ U R \cup R$. Otherwise, by separability,

$$
\begin{aligned}
B & \sim B^{\prime} \\
& =D R \cup(U R \cup R) \\
& \succsim D R \cup(D L \cup D) \\
& =A^{\prime} \\
& \sim A,
\end{aligned}
$$

contradicting $A \succ B$.

We claim $L \cup R \succsim U \cup D$. Otherwise, by separability,

$$
\begin{aligned}
A \cup A^{\prime} & =(U L \cup U R \cup D L \cup D R) \cup(U \cup D) \\
& \succ(U L \cup U R \cup D L \cup D R) \cup(L \cup R) \\
& =B \cup B^{\prime},
\end{aligned}
$$

contradicting $A \cup A^{\prime} \sim B \cup B^{\prime}$. Similarly, $U \cup D \succsim L \cup R$, so $L \cup R \sim U \cup D$.

But then by separability,

$$
\begin{aligned}
(L \cup R) \cup(U L \cup U R) & \sim(U \cup D) \cup(U L \cup U R) \\
& =A \cup D \\
& \succ B \cup D \\
& =(U L \cup L) \cup(D L \cup D) \\
& \succ(U L \cup L) \cup(U R \cup R),
\end{aligned}
$$

contradicting $L \cup R \cup U L \cup U R \sim L \cup R \cup U L \cup U R$.

The First Halving Lemma states any event satisfying 1- $A S$ can be associated with two disjoint subevents analogous to its halves:

- First Halving Lemma: If $(S, \mathcal{A}, \succsim)$ is a purely- $\sigma$-catalogued qualitative probability space satisfying (monotone) continuity, then for each $A \in \mathcal{A}$ such that $A \succ \emptyset$ and $\left.\succsim\right|_{A}$ satisfies $1-A S$, there is $H \subseteq A$ such that $H \sim A \backslash H$ and $A \succ H \succ \emptyset$.

Proof: Since $A \neq \emptyset$, by $1-A S,|A|=|\mathbb{N}|$. Assume, without loss of generality, $A=S=\mathbb{N}$. Let $\mathbb{C} \subseteq[0,1]$ be the canonical Cantor set (Cantor, 1883). Define $\Psi: \mathcal{A} \rightarrow \mathbb{C}$ by

$$
\Psi(B)=\sum_{s \in B} \frac{2 s}{3^{s}}
$$

It is well-known that $\Psi$ is an order-preserving homeomorphism when $\mathcal{A}$ has the lexicographic order $>_{\text {LEX }}$ and $\mathbb{C}$ has the usual order $>$; thus each closed collection $\mathcal{A}^{\prime} \subseteq \mathcal{A}$

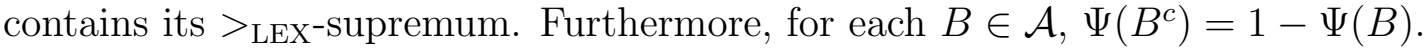

Define the collection of events $\mathcal{A}^{-} \subseteq \mathcal{A}$ by:

$$
\mathcal{A}^{-} \equiv\left\{B \in \mathcal{A} \mid B^{c} \succsim B\right\}
$$


We claim $\mathcal{A}^{-}$is closed. Indeed, let $\left(B_{i}\right) \in\left(\mathcal{A}^{-}\right)^{\mathbb{N}}$ be convergent and define $B \equiv \lim \left(B_{i}\right)$. Then

$$
\begin{aligned}
\lim \left(B_{i}^{c}\right) & =\lim \left(\Psi^{-1}\left(\Psi\left(B_{i}^{c}\right)\right)\right) \\
& =\lim \left(\Psi^{-1}\left(1-\Psi\left(B_{i}\right)\right)\right) \\
& =\Psi^{-1}\left(\lim \left(1-\Psi\left(B_{i}\right)\right)\right) \\
& =\Psi^{-1}\left(1-\lim \Psi\left(B_{i}\right)\right) \\
& =\Psi^{-1}(1-\Psi(B)) \\
& =B^{c} .
\end{aligned}
$$

Thus $\left(B_{i}\right),\left(B_{i}^{c}\right)$ are convergent such that for each $i \in \mathbb{N}, B_{i}^{c} \succsim B_{i}$, so by the LimitOrder Lemma,

$$
\begin{aligned}
\lim (B)^{c} & =B^{c} \\
& =\lim \left(B_{i}^{c}\right) \\
& \succsim \lim \left(B_{i}\right),
\end{aligned}
$$

and hence $\lim \left(B_{i}\right) \in \mathcal{A}^{-}$. Thus $\mathcal{A}^{-}$is closed, so it contains its $>_{\text {LEX }}$-supremum $H$.

Since $H=S$ implies $H \succ H^{c}$, contradicting $H \in \mathcal{A}^{-}$, thus $H^{c} \neq \emptyset$. Similarly, $H^{c} \neq S$. Assume, by way of contradiction, $\left|H^{c}\right|<|\mathbb{N}|$. Define $s^{*} \equiv \max H^{c}$. By $1-A S$ and separability,

$$
\begin{aligned}
H & =\left[H \backslash\left\{s^{*}+1, s^{*}+2, \ldots\right\}\right] \cup\left\{s^{*}+1, s^{*}+2, \ldots\right\} \\
& \succsim\left[H \backslash\left\{s^{*}+1, s^{*}+2, \ldots\right\}\right] \cup\left\{s^{*}\right\} .
\end{aligned}
$$

Then by the Complement Lemma and separability,

$$
\begin{aligned}
\left(\left[H \backslash\left\{s^{*}+1, s^{*}+2, \ldots\right\}\right] \cup\left\{s^{*}\right\}\right)^{c} & \succsim H^{c} \\
& \succsim H \\
& \succsim\left[H \backslash\left\{s^{*}+1, s^{*}+2, \ldots\right\}\right] \cup\left\{s^{*}\right\} .
\end{aligned}
$$

But then $\left[H \backslash\left\{s^{*}+1, s^{*}+2, \ldots\right\}\right] \cup\left\{s^{*}\right\} \in \mathcal{A}^{-}$, contradicting the > LEX-maximality of $H$. Thus $\left|H^{c}\right|=|\mathbb{N}|$.

Finally, for each $s \in H^{c}, H \cup\{s\} \notin \mathcal{A}^{-}$, else $H \cup\{s\}$ would contradict the $>_{\text {LEX }^{-}}$ maximality of $H$ in $\mathcal{A}^{-}$. Thus by the Limit-Order Lemma,

$$
\begin{aligned}
H & =\lim _{s \in H^{c}}(H \cup\{s\}) \\
& \succsim \lim _{s \in H^{c}}(H \cup\{s\})^{c} \\
& =H^{c},
\end{aligned}
$$

and thus $H \sim H^{c}$.

Since $A \succ \emptyset$, necessarily $A \succ H$, else $H \succsim A$ and $H^{c} \sim H \succsim A \succ \emptyset$, so by the Domination Lemma, $A \sim H \cup H^{c} \succ A$, contradicting $A \sim A$. Necessarily $H \succ \emptyset$, else $\emptyset \succsim H$ and $\emptyset \succsim H \sim H^{c}$, so by the Domination Lemma, $\emptyset \succsim H \cup H^{c} \sim A$, contradicting $A \succ \emptyset$. Thus $A \succ H \succ \emptyset$.

The Second Halving Lemma states that under 2-AS, each event can be associated with two disjoint events analogous to its halves: 
- Second Halving Lemma: If $(S, \mathcal{A}, \succsim)$ is a purely- $\sigma$-catalogued qualitative probability space satisfying (monotone) continuity and $2-A S$, then for each $A \in \mathcal{A}$ such that $A \succ \emptyset$, there are disjoint $H(A), H^{\prime}(A) \in \mathcal{A}$ such that

(1) $A \sim H(A) \cup H^{\prime}(A)$, and

(2) $A \succ H(A) \sim H^{\prime}(A) \succ \emptyset$.

Proof: Let $A \in \mathcal{A}$ such that $A \succ \emptyset$. Since $\succsim$ satisfies $1-A S$ and, by monotonicity, $S \succsim S \backslash A$, thus by the 1-AS Lemma, $\mathcal{G}^{S}(S \backslash A) \sim S \backslash A$. By the Complement Lemma,

(1) $S \succ S \backslash A \sim \mathcal{G}^{S}(S \backslash A)$, and

(2) $S \backslash \mathcal{G}^{S}(S \backslash A) \sim S \backslash(S \backslash A)=A \succ \emptyset$.

Since $\succsim$ satisfies $2-A S$, by the Greedy Removal Lemma, $\left.\succsim\right|_{S \backslash \mathcal{G}^{S}(S \backslash A)}$ satisfies $1-A S$. By the First Halving Lemma, there are disjoint $H(A), H^{\prime}(A) \subseteq S \backslash \mathcal{G}^{S}(S \backslash A)$ such that

(1) $A \sim S \backslash \mathcal{G}^{S}(S \backslash A)=H(A) \cup H^{\prime}(A)$, and

(2) $A \sim S \backslash \mathcal{G}^{S}(S \backslash A) \succ H(A) \sim H^{\prime}(A) \succ \emptyset$,

as desired.

\section{Appendix 5}

In this appendix, we prove the Supercabinet Construction Lemma and Theorem 1.

- Supercabinet Construction Lemma: If $(S, \mathcal{A}, \succsim)$ is a purely- $\sigma$-catalogued qualitative probability space satisfying (monotone) continuity and $3-A S$, then it has a supercabinet.

Proof: The only notation carried from one step to the next is the notation in the step's statement.

○ Step 1: Define $\left\{A_{q}^{1}\right\}_{q \in\{0,1, \ldots\}},\left\{H\left(A_{q}^{1}\right)\right\}_{q \in\{0,1, \ldots\}},\left\{H^{\prime}\left(A_{q}^{1}\right)\right\}_{q \in\{0,1, \ldots\}} \subseteq \mathcal{A}$ such that for each $q \in\{0,1, \ldots\}$,

(1) $H\left(A_{q}^{1}\right) \cap H^{\prime}\left(A_{q}^{1}\right)=\emptyset$,

(2) $A_{q}^{1} \sim H\left(A_{q}^{1}\right) \cup H^{\prime}\left(A_{q}^{1}\right)$, and

(3) $A_{q}^{1} \succ A_{q+1}^{1} \sim H\left(A_{q}^{1}\right) \sim H^{\prime}\left(A_{q}^{1}\right) \succ \emptyset$.

We proceed recursively. Define $A_{0}^{1} \equiv \mathcal{G}^{S}(S)=S$; by monotonicity and nondegeneracy, $A_{0}^{1} \succ \emptyset$.

Suppose we have $A_{q}^{1} \in \mathcal{A}$ such that $A_{q}^{1} \succ \emptyset$. Since $\succsim$ satisfies $2-A S$, by the Second Halving Lemma, there are disjoint $H\left(A_{q}^{1}\right), H^{\prime}\left(A_{q}^{1}\right)$ such that

(1) $A_{q}^{1} \sim H\left(A_{q}^{1}\right) \cup H^{\prime}\left(A_{q}^{1}\right)$, and

(2) $A_{q}^{1} \succ H\left(A_{q}^{1}\right) \sim H^{\prime}\left(A_{q}^{1}\right) \succ \emptyset$.

Define $A_{q+1}^{1} \equiv \mathcal{G}^{S}\left(H\left(A_{q}^{1}\right)\right)$; by the Half-Equivalence Lemma this is well-defined. Since $\succsim$ satisfies $1-A S$ and, by monotonicity, $S \succsim H\left(A_{q}^{1}\right)$, thus by the 1-AS Lemma, $A_{q+1}^{1} \sim$ $H\left(A_{q}^{1}\right)$.

○ Step 2: For each $q \in\{0,1, \ldots\}$, define $\left\{A_{q}^{p}\right\}_{q \in\left\{0,1, \ldots, 2^{q}\right\}} \subseteq \mathcal{A}$ such that for each $p \in$ $\left\{0,1, \ldots, 2^{q}-1\right\}$

$$
A_{q}^{p+1} \sim A_{q}^{p} \cup \mathcal{G}^{S \backslash A_{q}^{p}}\left(A_{q}^{1}\right)
$$


We proceed recursively. For each $q \in \mathbb{N}$, define $A_{q}^{0} \equiv \emptyset$. For each $q \in\{0,1, \ldots\}$ and each $p \in\left\{0,1, \ldots, 2^{q}-1\right\}$, define

$$
A_{q}^{p+1} \equiv \mathcal{G}^{S}\left(A_{q}^{p} \cup \mathcal{G}^{S \backslash A_{q}^{p}}\left(A_{q}^{1}\right)\right)
$$

Since $\succsim$ satisfies $1-A S$ and, by monotonicity, $S \succsim A_{q}^{p} \cup \mathcal{G}^{S \backslash A_{q}^{p}}\left(A_{q}^{1}\right)$, thus by the 1-AS Lemma, $A_{q}^{p+1} \sim A_{q}^{p} \cup \mathcal{G}^{S \backslash A_{q}^{p}}\left(A_{q}^{1}\right)$.

By the Idempotence Lemma, this definition gives the same $\left\{A_{q}^{1}\right\}_{q \in\{0,1, \ldots\}}$ defined before.

○ STEP 3: For each $q \in\{0,1, \ldots\}$ and each $p \in\left\{0,1, \ldots, 2^{q}\right\},\left.\succsim\right|_{S \backslash A_{q}^{p}}$ satisfies $2-A S$.

Let $q \in\{0,1, \ldots\}$ and $p \in\left\{0,1, \ldots, 2^{q}\right\}$ be such that $A_{q}^{p} \neq S$. Since $\succsim$ satisfies $3-A S$, thus by the Greedy Removal Lemma and the Idempotence Lemma, $\left.\succsim\right|_{S \backslash A_{q}^{p}}=\left.\succsim\right|_{S \backslash \mathcal{G}^{S}\left(A_{q}^{p}\right)}$ satisfies $2-A S$.

○ SteP 4: For each $q \in\{0,1, \ldots\}$,

(1) $p \in\left\{0,1, \ldots, 2^{q}-1\right\}$ implies $\mathcal{G}^{S \backslash A_{q}^{p}}\left(A_{q}^{1}\right) \sim A_{q}^{1}$, and

(2) $p \in\left\{0,1, \ldots, 2^{q}\right\}$ implies $A_{q}^{p}=A_{q+1}^{2 p}$.

We proceed by induction on $q$. For the base step, let $q=0$. Then $\mathcal{G}^{S \backslash A_{q}^{0}}\left(A_{q}^{1}\right)=$ $\mathcal{G}^{S}(S)=S=A_{q}^{1}$. For the inductive hypothesis, assume $q \in\{0,1, \ldots\}$ is such that for each $p \in\left\{0,1, \ldots, 2^{q}-1\right\}, \mathcal{G}^{S \backslash A_{q}^{p}}\left(A_{q}^{1}\right) \sim A_{q}^{1}$.

Within the current inductive argument, we make a second inductive argument, on $p$. For the base step, $A_{q}^{0}=\emptyset=A_{q+1}^{0}$. For the inductive hypothesis, assume $p \in\left\{0,1, \ldots, 2^{q}-\right.$ $1\}$ is such that $A_{q}^{p}=A_{q+1}^{2 p}$. For convenience, define $G_{1}, G_{2}, G_{2}^{\prime} \in \mathcal{A}$ by

- $G_{1} \equiv \mathcal{G}^{S \backslash A_{q+1}^{2 p}}\left(A_{q+1}^{1}\right)$,

- $G_{2} \equiv \mathcal{G}^{S \backslash A_{q+1}^{2 p+1}}\left(A_{q+1}^{1}\right)$, and

- $G_{2}^{\prime} \equiv \mathcal{G}^{S \backslash\left[A_{q+1}^{2 p} \cup G_{1}\right]}\left(A_{q+1}^{1}\right)$.

We make three claims, which we prove in sequence:

Claim 1: $G_{1} \sim A_{q+1}^{1}$,

Claim 2: $G_{2} \sim A_{q+1}^{1}$, and

Claim 3: $A_{q}^{p+1}=A_{q+1}^{2(p+1)}$.

Proof of Claim 1: By the inductive hypothesis on $p, A_{q}^{p}=A_{q+1}^{2 p}$, so by Step 1 , monotonicity, and the inductive hypothesis on $q$,

$$
\begin{aligned}
S \backslash A_{q+1}^{2 p} & =S \backslash A_{q}^{p} \\
& \succsim \mathcal{G}^{S \backslash A_{q}^{p}}\left(A_{q}^{1}\right) \\
& \sim A_{q}^{1} \\
& \succ A_{q+1}^{1} .
\end{aligned}
$$


Since by Step $3,\left.\succsim\right|_{S \backslash A_{q+1}^{2 p}}=\left.\succsim\right|_{S \backslash A^{p}}$ satisfies 2 -AS, thus by the 1-AS Lemma, $G_{1}=$ $\mathcal{G}^{S \backslash A_{q+1}^{2 p}}\left(A_{q+1}^{1}\right) \sim A_{q+1}^{1}$.

Proof of Claim 2: Since by Claim 1, $H\left(A_{q}^{1}\right) \sim A_{q+1}^{1} \sim G_{1}$, necessarily $S \backslash\left[A_{q+1}^{2 p} \cup\right.$ $\left.G_{1}\right] \succsim H^{\prime}\left(A_{q}^{1}\right)$, else by the Domination Lemma, the hypothesis on $p$, and the hypothesis on $q$,

$$
\begin{aligned}
A_{q}^{1} & \sim H\left(A_{q}^{1}\right) \cup H^{\prime}\left(A_{q}^{1}\right) \\
& \succ G_{1} \cup S \backslash\left[A_{q+1}^{2 p} \cup G_{1}\right] \\
& =S \backslash A_{q+1}^{2 p} \\
& =S \backslash A_{q}^{p} \\
& \succsim \mathcal{G}^{S \backslash A_{q}^{p}}\left(A_{q}^{1}\right) \\
& \sim A_{q}^{1},
\end{aligned}
$$

contradicting $A_{q}^{1} \sim A_{q}^{1}$.

By Step 2, $A_{q+1}^{2 p+1} \sim A_{q+1}^{2 p} \cup G_{1}$. By the Complement Lemma, $S \backslash A_{q+1}^{2 p+1} \sim S \backslash\left[A_{q+1}^{2 p} \cup\right.$ $\left.G_{1}\right] \succsim H^{\prime}\left(A_{q}^{1}\right) \sim A_{q+1}^{1}$. By Step $3,\left.\succsim\right|_{S \backslash A_{q+1}^{2 p+1}}$ satisfies $2-A S$, so by the 1-AS Lemma, $G_{2}=\mathcal{G}^{S \backslash A_{q+1}^{2 p+1}}\left(A_{q+1}^{1}\right) \sim A_{q+1}^{1}$.

Proof of Claim 3: As argued in the proof of Claim 2, $S \backslash\left[A_{q+1}^{2 p} \cup G_{1}\right] \succsim H^{\prime}\left(A_{q}^{1}\right) \sim$ $A_{q+1}^{1}$. By Step $3,\left.\succsim\right|_{S \backslash A_{q+1}^{2 p}}$ satisfies $2-A S$, so by the Greedy Removal Lemma, $\left.\succsim\right|_{\left(S \backslash A_{q+1}^{2 p}\right) \backslash G_{1}}=$ $\left.\succsim\right|_{S \backslash\left[A_{q+1}^{2 p} \cup G_{1}\right]}$ satisfies 1-AS. Thus by the 1-AS Lemma, $G_{2}^{\prime} \sim A_{q+1}^{1}$.

Since $H\left(A_{q}^{1}\right) \sim G_{1}$ and $H^{\prime}\left(A_{q}^{1}\right) \sim G_{2}^{\prime}$, thus by the Domination Lemma, $A_{q}^{1} \sim H\left(A_{q}^{1}\right) \cup$ $H^{\prime}\left(A_{q}^{1}\right) \sim G^{1} \cup G_{2}^{\prime}$. By the hypothesis on $q, \mathcal{G}^{S \backslash A_{q}^{p}}\left(A_{q}^{1}\right) \sim A_{q}^{1} \sim G^{1} \cup G_{2}^{\prime}$. By Step 2, the hypothesis on $p$, and separability,

$$
\begin{aligned}
A_{q}^{p+1} & \sim A_{q}^{p} \cup \mathcal{G}^{S \backslash A_{q}^{p}}\left(A_{q}^{1}\right) \\
& =A_{q+1}^{2 p} \cup \mathcal{G}^{S \backslash A_{q}^{p}}\left(A_{q}^{1}\right) \\
& \sim A_{q+1}^{2 p} \cup\left(G_{1} \cup G_{2}^{\prime}\right) \\
& =\left[A_{q+1}^{2 p} \cup G_{1}\right] \cup G_{2}^{\prime}
\end{aligned}
$$

By Step 2, $A_{q+1}^{2 p} \cup G_{1} \sim A_{q+1}^{2 p+1}$. By the first paragraph of this claim's proof and Claim 2, $G_{2}^{\prime} \sim A_{q+1}^{1} \sim G_{2}$. Thus by the Domination Lemma, $\left[A_{q+1}^{2 p} \cup G_{1}\right] \cup G_{2}^{\prime} \sim A_{q+1}^{2 p+1} \cup G_{2}$. By Step 2, $A_{q+1}^{2 p+1} \cup G_{2} \sim A_{q+1}^{2 p+2}$. Altogether, $A_{q}^{p+1} \sim A_{q+1}^{2 p+2}$.

By induction on $p$, we conclude the following: if $q \in\{0,1, \ldots\}$ is such that for each $p \in\left\{0,1, \ldots, 2^{q}-1\right\}, \mathcal{G}^{S \backslash A_{q}^{p}}\left(A_{q}^{1}\right) \sim A_{q}^{1}$, then

(1) for each $p \in\left\{0,1, \ldots, 2^{q+1}-1\right\}, \mathcal{G}^{S \backslash A_{q+1}^{p}}\left(A_{q+1}^{1}\right) \sim A_{q+1}^{1}$, and

(2) for each $p \in\left\{0,1, \ldots, 2^{q}\right\}, A_{q}^{p}=A_{q+1}^{2 p}$.

By induction on $q$, we are done.

○ STEP 5: For each $q \in\{0,1, \ldots\}$ and each $p \in\left\{0,1, \ldots, 2^{q}\right\}, A_{q}^{p} \sim S \backslash A_{q}^{2^{q}}-p$.

Let $q \in\{0,1, \ldots\}$. For each $p \in\left\{0,1, \ldots, 2^{q}\right\}$, define $B_{q}^{p} \equiv S \backslash A_{q}^{2 q-p}$. We proceed by induction on $p$. 
For the base step, let $p=0$. By Step $4, B_{q}^{p}=S \backslash A_{q}^{2 q}=S \backslash A_{0}^{1}=S \backslash S=\emptyset$, so $A_{q}^{0} \sim B_{q}^{0}$. For the inductive hypothesis, assume $p \in\left\{0,1, \ldots, 2^{q}-1\right\}$ is such that $A_{q}^{p} \sim B_{q}^{p}$.

Define $A, B, C^{\prime}, B^{\prime}, A^{\prime} \in \mathcal{A}$ by:

$$
\begin{aligned}
A & \equiv A_{q}^{p}, \\
B & \equiv \mathcal{G}^{S \backslash A}\left(A_{q}^{1}\right), \\
C^{\prime} & \equiv A_{q}^{2^{q}-(p+1)} \\
B^{\prime} & \equiv \mathcal{G}^{S \backslash C^{\prime}}\left(A_{q}^{1}\right) \\
A^{\prime} & \equiv S \backslash\left(C^{\prime} \cup B^{\prime}\right)
\end{aligned}
$$

By Step 2, $C^{\prime} \cup B^{\prime} \sim A_{q}^{2 q-p}$. By the Complement Lemma, $A^{\prime}=S \backslash\left(C^{\prime} \cup B^{\prime}\right) \sim$ $S \backslash A_{q}^{2 q-p}=B_{q}^{p}$. By the hypothesis on $p, A^{\prime} \sim A_{q}^{p}=A$. By Step $4, B \sim A_{q}^{1} \sim B^{\prime}$. Thus by the Domination Lemma, $A \cup B \sim A^{\prime} \cup B^{\prime}$.

By Step 2, $A \cup B \sim A_{q}^{p+1}$, and by definition, $A^{\prime} \cup B^{\prime}=S \backslash A_{q}^{2^{q}-(p+1)}=B_{q}^{p+1}$, so altogether $A_{q}^{p+1} \sim B_{q}^{p+1}$. By induction on $p$, we are done.

○ Step 6: Define the binary operation $\biguplus$.

For each $q \in\{0,1, \ldots\}$ and each pair $p, p^{\prime} \in\left\{0,1, \ldots, 2^{q}\right\}$ such that $p+p^{\prime} \leq 2^{q}$, define $A_{q}^{p} \biguplus A_{q}^{p^{\prime}} \in \mathcal{A}$ by:

$$
A_{q}^{p} \biguplus A_{q}^{p^{\prime}} \equiv \mathcal{G}^{S}\left(A_{q}^{p} \cup \mathcal{G}^{S \backslash A_{q}^{p}}\left(A_{q}^{p^{\prime}}\right)\right)
$$

○ STEP 7: For each $q \in\{0,1, \ldots\}$ and each pair $p, p^{\prime} \in\left\{0,1, \ldots, 2^{q}\right\}$ such that $p+p^{\prime} \leq 2^{q}$,

$$
A_{q}^{p} \biguplus A_{q^{\prime}}^{p^{\prime}}=A_{q}^{p+p^{\prime}} .
$$

Let $q \in\{0,1, \ldots\}$ and let $p \in\left\{0,1, \ldots, 2^{q}\right\}$. We proceed by induction on $p^{\prime}$. For the base step, let $p^{\prime}=0$. Then by the Idempotence Lemma, $A_{q}^{p} \biguplus A_{q}^{p^{\prime}}=\mathcal{G}^{S}\left(A_{q}^{p}\right)=A_{q}^{p}$.

For the inductive hypothesis, assume $p^{\prime} \in\left\{0,1, \ldots,\left[2^{q}-1\right]-p\right\}$ is such that $A_{q}^{p} \biguplus A_{q}^{p^{\prime}}=$ $A_{q}^{p+p^{\prime}}$. Define $A, B, C \in \mathcal{A}$ by:

$$
\begin{aligned}
A & \equiv A_{q}^{p}, \\
B & \equiv \mathcal{G}^{S \backslash A}\left(A_{q}^{p^{\prime}}\right), \text { and } \\
C & \equiv \mathcal{G}^{S \backslash(A \cup B)}\left(A_{q}^{1}\right) .
\end{aligned}
$$

By hypothesis, $A_{q}^{p+p^{\prime}}=A_{q}^{p} \biguplus A_{q}^{p^{\prime}}=\mathcal{G}^{S}(A \cup B)$. Since $\succsim$ satisfies $1-A S$ and, by monotonicity, $S \succsim A \cup B$, thus by the 1-AS Lemma, $\mathcal{G}^{S}(A \cup B) \sim A \cup B$. Altogether, $A_{q}^{p+p^{\prime}} \sim A \cup B$.

By monotonicity and Step $4, S \backslash A_{q}^{p+p^{\prime}} \succsim \mathcal{G}^{S \backslash A_{q}^{p+p^{\prime}}}\left(A_{q}^{1}\right) \sim A_{q}^{1}$, so by the Complement Lemma, $S \backslash(A \cup B) \sim S \backslash A_{q}^{p+p^{\prime}} \succsim A_{q}^{1}$. By Step 3, $\left.\right|_{S \backslash A}$ satisfies $2-A S$, so by the Greedy Removal Lemma, $\succsim_{(S \backslash A) \backslash B}=\succsim_{S \backslash(A \cup B)}$ satisfies 1 - $A S$. Thus by the 1-AS Lemma, $A_{q}^{1} \sim C$. By Step $4, \mathcal{G}^{S \backslash A_{q}^{p+p^{\prime}}}\left(A_{q}^{1}\right) \sim A_{q}^{1}$, so $\mathcal{G}^{S \backslash A_{q}^{p+p^{\prime}}}\left(A_{q}^{1}\right) \sim C$. 
Since $A_{q}^{p+p^{\prime}} \sim A \cup B$ and $\mathcal{G}^{S \backslash A_{q}^{p+p^{\prime}}}\left(A_{q}^{1}\right) \sim C$, thus by Step 2 and the Domination Lemma,

$$
\begin{aligned}
A_{q}^{p+p^{\prime}+1} & \sim A_{q}^{p+p^{\prime}} \cup \mathcal{G}^{S \backslash A_{q}^{p+p^{\prime}}}\left(A_{q}^{1}\right) \\
& \sim(A \cup B) \cup C .
\end{aligned}
$$

By Step 5, $S \backslash A_{q}^{p} \sim A_{q}^{2^{q}-p}$. Since $2^{q}-p \geq p^{\prime}+1$, by Step 2 and monotonicity, $A_{q}^{2^{q}-p} \succsim A_{q}^{p^{\prime}+1}$, so $S \backslash A_{q}^{p} \succsim A_{q}^{p^{\prime}+1}$. By Step $3,\left.\succsim\right|_{S \backslash A_{q}^{p}}$ satisfies 2 -AS, so by the 1 -AS Lemma, $\mathcal{G}^{S \backslash A_{q}^{p}}\left(A_{q}^{p^{\prime}+1}\right) \sim A_{q}^{p^{\prime}+1}$.

Since $S \backslash A_{q}^{p} \succsim A_{q}^{p^{\prime}+1}$, by Step 2 and monotonicity, $S \backslash A_{q}^{p} \succsim A_{q}^{p^{\prime}}$. By Step $3,\left.\succsim\right|_{S \backslash A_{q}^{p}}$ satisfies $2-A S$, so by the 1-AS Lemma, $A_{q}^{p^{\prime}} \sim B$. By Step $4, \mathcal{G}^{S \backslash A_{q}^{p^{\prime}}}\left(A_{q}^{1}\right) \sim A_{q}^{1}$, and as argued above, $A_{q}^{1} \sim C$, so $\mathcal{G}^{S \backslash A_{q}^{p^{\prime}}}\left(A_{q}^{1}\right) \sim C$. Thus by Step 2 and the Domination Lemma, $A_{q}^{p^{\prime}+1} \sim A_{q}^{p^{\prime}} \cup \mathcal{G}^{S \backslash A_{q}^{p^{\prime}}}\left(A_{q}^{1}\right) \sim B \cup C$.

Altogether, $\mathcal{G}^{S \backslash A_{q}^{p}}\left(A_{q}^{p^{\prime}+1}\right) \sim B \cup C$. Since $\succsim$ satisfies $1-A S$ and, by monotonicity, $S \succsim A_{q}^{p} \cup \mathcal{G}^{S \backslash A_{q}^{p}}\left(A_{q}^{p^{\prime}+1}\right)$, thus by the 1-AS Lemma and separability,

$$
\begin{aligned}
A_{q}^{p} \biguplus A_{q}^{p^{\prime}+1} & =\mathcal{G}^{S}\left(A_{q}^{p} \cup \mathcal{G}^{S \backslash A_{q}^{p}}\left(A_{q}^{p^{\prime}+1}\right)\right) \\
& \sim A_{q}^{p} \cup \mathcal{G}^{S \backslash A_{q}^{p}}\left(A_{q}^{p^{\prime}+1}\right) \\
& \sim A_{q}^{p} \cup(B \cup C) \\
& =A \cup(B \cup C) .
\end{aligned}
$$

Since $(A \cup B) \cup C=A \cup(B \cup C)$, thus $A_{q}^{p+p^{\prime}+1} \sim A_{q}^{p} \biguplus A_{q}^{p^{\prime}+1}$. By the Idempotence Lemma, $A_{q}^{p+p^{\prime}+1}=A_{q}^{p} \biguplus A_{q}^{p^{\prime}+1}$.

By induction on $p^{\prime}$, for each $p^{\prime} \in\left\{0,1, \ldots, 2^{q}-1\right\}, A_{q}^{p} \biguplus A_{q}^{p^{\prime}}=A_{q}^{p+p^{\prime}}$. Since $q \in\{0,1, \ldots\}$ and $p \in\left\{0,1, \ldots, 2^{q}\right\}$ were arbitrary, we are done.

○ STEP 8: $\lim A_{q}^{1}=\emptyset$.

By Step 2, monotonicity, and the Convergence Lemma, $\left(A_{q}^{1}\right) \in \mathcal{A}^{\mathbb{N}}$ is convergent. Assume, by way of contradiction, there is $s \in \lim A_{q}^{1}$.

We claim for each $i \in \mathbb{N}$ and each $k \in \mathbb{N}, A_{i}^{1} \succ\{s, s+1, \ldots, s+k\}$. We proceed by induction on $k$. For the base step, let $k=0$. Then by continuity and monotonicity, for each $i \in \mathbb{N}, A_{i}^{1} \succsim \lim A_{q}^{1} \succsim\{s\}$, so by Step $1, A_{i}^{1} \succ A_{i+1}^{1} \succsim\{s\}$.

For the inductive hypothesis, assume $k \in \mathbb{N}$ is such that for each $i \in \mathbb{N}, A_{i}^{1} \succ$ $\{s, s+1, \ldots, s+k\}$. Let $i \in \mathbb{N}$. By Step 1, $H\left(A_{i}^{1}\right) \sim A_{i+1}^{1}$. By Step 4 and Step 1, $H^{\prime}\left(A_{i+1}^{1}\right) \sim A_{i+1}^{1} \sim \mathcal{G}^{S \backslash A_{i+1}^{1}}\left(A_{i+1}^{1}\right)$. By the inductive hypothesis, $A_{i+1}^{1} \succ\{s, s+1, \ldots, s+k\}$. By Step 4 and the inductive hypothesis, $\mathcal{G}^{S \backslash A_{i+1}^{1}}\left(A_{i+1}^{1}\right) \sim A_{i+1}^{1} \succ\{s\} \succsim\{s+k+1\}$. Thus by Step 1 and two applications of the Domination Lemma,

$$
\begin{aligned}
A_{i}^{1} & \sim H\left(A_{i}^{1}\right) \cup H^{\prime}\left(A_{i}^{1}\right) \\
& \sim A_{i+1}^{1} \cup \mathcal{G}^{S \backslash A_{i+1}^{1}}\left(A_{i+1}^{1}\right) \\
& \succ\{s, s+1, \ldots, s+k\} \cup\{s+k+1\} .
\end{aligned}
$$

Since $i \in \mathbb{N}$ was arbitrary, for each $i \in \mathbb{N}, A_{i}^{1} \succ\{s, s+1, \ldots, s+k+1\}$. By induction on $k$, we are done. 
Thus by continuity, for each $i \in \mathbb{N}, A_{i}^{1} \succsim \lim \{s, s+1, \ldots, s+k\}=\{s, s+1, \ldots\}$. If $s=1$, then by Step $1, S=A_{0}^{1} \succ A_{1}^{1} \succsim\{1,2, \ldots\}=S$, contradicting $S \sim S$. Thus $s>1$, so by $2-A S$, for each $i \in \mathbb{N}, A_{i}^{1} \succ\{s, s+1, \ldots\} \succsim\{s-1\}$.

Thus for each $s \in S$, if for each $i \in \mathbb{N}, A_{i}^{1} \succ\{s\}$, then (1) $s \neq 1$, and (2) for each $i \in \mathbb{N}, A_{i}^{1} \succ\{s-1\}$. Then there can be no such $s$, contradicting that there is.

○ Step 9: Define $\left\{A_{v}\right\}_{v \in \mathbb{L}} \subseteq \mathcal{A}$ and $\left\{\mathcal{Z}_{v}\right\}_{v \in \mathcal{L}} \subseteq[\mathcal{A} / \sim]$ such that for each pair $v, v^{\prime} \in \mathcal{Z}$ such that $v^{\prime}>v, A_{v^{\prime}} \succ A_{v}$.

Let $v \in \mathcal{Z}$. Then there are $p, q \in\{0,1, \ldots\}$ such that $p \leq 2^{q}$ and $v=\frac{p}{2^{q}}$. Define

$$
\begin{aligned}
& A_{v} \equiv A_{q}^{p}, \text { and } \\
& \mathcal{Z}_{v} \equiv\left\{A \in \mathcal{A} \mid A \sim A_{v}\right\} .
\end{aligned}
$$

By Step 4, this is well-defined.

Let $v, v^{\prime} \in \mathbb{2}$ such that $v^{\prime}>v$. Since $v^{\prime}-v>0$, by Step 1, Step 2, and monotonicity, $A_{v^{\prime}-v} \succ \emptyset$. Similarly, $A_{1-v} \succ \emptyset$. By Step 5, $S \backslash A_{v} \sim A_{1-v} \succ \emptyset$, so $\mathcal{G}^{S \backslash A_{v}}\left(A_{v^{\prime}-v}\right) \succ \emptyset$. Since $\succsim$ satisfies $1-A S$ and, by monotonicity, $S \succsim A_{v} \cup \mathcal{G}^{S \backslash A_{v}}\left(A_{v^{\prime}-v}\right)$, thus by the 1-AS Lemma, $\mathcal{G}^{S}\left(A_{v} \cup \mathcal{G}^{S \backslash A_{v}}\left(A_{v^{\prime}-v}\right)\right) \sim A_{v} \cup \mathcal{G}^{S \backslash A_{v}}\left(A_{v^{\prime}-v}\right)$. Thus by Step $6, A_{v^{\prime}}=\mathcal{G}^{S}\left(A_{v} \cup\right.$ $\left.\mathcal{G}^{S \backslash A_{v}}\left(A_{v^{\prime}-v}\right)\right) \sim A_{v} \cup \mathcal{G}^{S \backslash A_{v}}\left(A_{v^{\prime}-v}\right)$, so by separability, $A_{v^{\prime}} \succ A_{v}$.

○ STEP 10: For each convergent pair $\left(v_{i}\right),\left(v_{i}^{\prime}\right) \in \mathcal{2}^{\mathbb{N}} \operatorname{such}$ that $\lim v_{i}=\lim v_{i}$, if $\left(A_{v_{i}}\right),\left(A_{v_{i}^{\prime}}\right) \in$ $\mathcal{A}^{\mathbb{N}}$ are convergent, then $\lim A_{v_{i}} \sim \lim A_{v_{i}^{\prime}}$.

Define $v_{\infty} \equiv \lim v_{i}=\lim v_{i}^{\prime}, A_{\infty} \equiv \lim A_{v_{i}}$, and $A_{\infty}^{\prime} \equiv \lim A_{v_{i}^{\prime}}$. Let $v \in \mathbb{2}$. If $v>v_{\infty}$, then there is $v^{\prime} \in \mathbb{Z}$ such that $v>v^{\prime}>v_{\infty}$. Since $\lim v_{i}=v_{\infty}$, there is $i^{*} \in \mathbb{N}$ such that for each $i \geq i^{*}, v^{\prime}>v_{i}$. Thus for each $i \geq i^{*}, A_{v^{\prime}} \succ A_{v_{i}}$, so by Step 9 and continuity, $A_{v} \succ A_{v^{\prime}} \succsim A_{\infty}$. By the same argument, $v>v_{\infty}$ implies $A_{v} \succ A_{\infty}^{\prime}$.

Similarly, for each $v \in \mathcal{Z}, v_{\infty}>v$ implies $A_{\infty} \succ A_{v}$ and $A_{\infty}^{\prime} \succ A_{v}$.

Assume, by way of contradiction, $A_{\infty} \nsim A_{\infty}^{\prime}$. Assume, without loss of generality, $A_{\infty} \succ A_{\infty}^{\prime}$. Define $G \equiv \mathcal{G}^{S}\left(A_{\infty}^{\prime}\right)$. Since $\succsim$ satisfies $1-A S$ and, by monotonicity, $S \succsim A_{\infty}^{\prime}$, thus by the 1-AS Lemma, $A_{\infty} \succ A_{\infty}^{\prime} \sim G$.

Necessarily $S \backslash G \succ \emptyset$, else by the Complement Lemma and monotonicity, $G \succsim S \succsim$ $A_{\infty}$, contradicting $A_{\infty} \succ G$. Then there is $q \in \mathbb{N}$ such that $S \backslash G \succ A_{q}^{1}$, else by Step 8 and continuity, $\emptyset=\lim A_{q}^{1} \succsim S \backslash G$, contradicting $S \backslash G \succ \emptyset$.

Since $\succsim$ satisfies $3-A S$, thus by the Greedy Removal Lemma, $\left.\succsim\right|_{S \backslash G}$ satisfies $2-A S$, so by Step 1 and the 1 -AS Lemma, for each $q^{\prime} \in \mathbb{N}$ such that $q^{\prime} \geq q, \mathcal{G}^{S \backslash G}\left(A_{q^{\prime}}^{1}\right) \sim A_{q^{\prime}}^{1}$. Then by Step $1,\left(\mathcal{G}^{S \backslash G}\left(A_{q^{\prime}}^{1}\right)\right) \in \mathcal{A}^{\mathbb{N}}$ is monotonic, so by the Convergence Lemma, it is convergent. By the Limit-Order Lemma and Step 8, $\lim \mathcal{G}^{S \backslash G}\left(A_{q^{\prime}}^{1}\right) \sim \lim A_{q^{\prime}}^{1}=\emptyset$, so $\lim \mathcal{G}^{S \backslash G}\left(A_{q^{\prime}}^{1}\right)=\emptyset$. Then $\lim G \cup \mathcal{G}^{S \backslash G}\left(A_{q^{\prime}}^{1}\right)=G$. Thus there is $q^{*} \in \mathbb{N}$ such that (1) $q^{*} \geq q$ and thus $\mathcal{G}^{S \backslash G}\left(A_{q^{*}}^{1}\right) \sim A_{q^{*}}^{1}$, and (2) $A_{\infty} \succ G \cup \mathcal{G}^{S \backslash G}\left(A_{q^{*}}^{1}\right)$, else by continuity $G \succsim A_{\infty}$, contradicting $A_{\infty} \succ G$.

We proceed by reaching a contradiction in three cases:

CASE 1: $v_{\infty}=0$. Then since $\frac{1}{2^{q^{*}}}>v_{\infty}$, by monotonicity, $A_{q^{*}}^{1} \succ A_{\infty} \succ G \cup$ $\mathcal{G}^{S \backslash G}\left(A_{q^{*}}^{1}\right) \succsim \mathcal{G}^{S \backslash G}\left(A_{q^{*}}^{1}\right) \sim A_{q^{*}}^{1}$, contradicting $A_{q^{*}}^{1} \sim A_{q^{*}}^{1}$. 
CASE 2: $v_{\infty}=1$. Since $v_{\infty}>\frac{2^{q^{*}}-1}{2^{q^{*}}}, G \sim A_{\infty}^{\prime} \succ A_{q^{*}}^{2^{q^{*}}-1}$. Since $\mathcal{G}^{S \backslash G}\left(A_{q^{*}}^{1}\right) \sim$ $A_{q^{*}}^{1} \succsim \mathcal{G}^{S \backslash A_{q^{*}}^{2^{*}}-1}\left(A_{q^{*}}^{1}\right)$, thus by the Domination Lemma, $A_{\infty} \succ G \cup \mathcal{G}^{S \backslash G}\left(A_{q^{*}}^{1}\right) \succ A_{q^{*}}^{2^{*}-1} \cup$ $\mathcal{G}^{S \backslash A_{q^{*}}^{2^{*}-1}}\left(A_{q^{*}}^{1}\right)$. Since $\succsim$ satisfies 1-AS and, by monotonicity, $S \succsim A_{q^{*}}^{2^{*}-1} \cup \mathcal{G}^{S \backslash A_{q^{*}}^{q^{*}-1}}\left(A_{q^{*}}^{1}\right)$, thus by the 1-AS Lemma, $A_{q^{*}}^{2^{*}-1} \cup \mathcal{G}^{S \backslash A_{q^{*}}^{2^{q^{*}}-1}}\left(A_{q^{*}}^{1}\right) \sim A_{q^{*}}^{2^{*}-1} \biguplus A_{q^{*}}^{1}$. But then by Step 7, Step 4, and Step 1, $A_{\infty} \succ A_{q^{*}}^{2^{*}-1} \biguplus A_{q^{*}}^{1}=A_{q^{*}}^{2^{*}}=S$, contradicting $S \succsim A_{\infty}$.

CASE 3: $v_{\infty} \in(0,1)$. Define $\epsilon^{*} \equiv \frac{1}{2^{q^{*}}}$. Since $\mathcal{L}$ is dense in $[0,1]$, there is $v^{*} \in \mathbb{Z}$ such that $1 \geq v^{*}+\epsilon^{*}>v_{\infty}>v^{*}$. Then $A_{v^{*}+\epsilon^{*}} \succ A_{\infty} \succ G \sim A_{\infty}^{\prime} \succ A_{v^{*}}$.

Since $1-v^{*} \geq \epsilon^{*}$, thus by Step $5, S \backslash A_{v^{*}} \sim A_{1-v^{*}} \succsim A_{\epsilon^{*}}=A_{q^{*}}^{1}$. Then by Step 3 and the 1-AS Lemma, $\mathcal{G}^{S \backslash G}\left(A_{q^{*}}^{1}\right) \sim A_{q^{*}}^{1} \sim \mathcal{G}^{S \backslash A_{v^{*}}}\left(A_{q^{*}}^{1}\right)$. Since $G \succ A_{v^{*}}$, thus by the Domination Lemma, $A_{\infty} \succ G \cup \mathcal{G}^{S \backslash G}\left(A_{q^{*}}^{1}\right) \succ A_{v^{*}} \cup \mathcal{G}^{S \backslash A_{v^{*}}}\left(A_{q^{*}}^{1}\right)$.

Since $\succsim$ satisfies 1-AS and, by monotonicity, $S \succsim A_{v^{*}} \cup \mathcal{G}^{S \backslash A_{v^{*}}}\left(A_{q^{*}}^{1}\right)$, thus by the 1-AS Lemma, $A_{v^{*}} \biguplus A_{\epsilon^{*}}=A_{v^{*}} \cup \mathcal{G}^{S \backslash A_{v^{*}}}\left(A_{q^{*}}^{1}\right)$. But then by Step 7, $A_{\infty} \succ A_{v^{*}} \biguplus A_{\epsilon^{*}}=A_{v^{*}+\epsilon^{*}}$, contradicting $A_{v^{*}+\epsilon^{*}} \succ A_{\infty}$.

- SteP 11: Conclude.

We verify that $\left\{\mathcal{Z}_{v}\right\}_{v \in \mathbb{2}}$ satisfies [SC1], [SC2], [SC3], and [SC4].

SC1: By Step 1 and Step $9, \emptyset=A_{0} \in \mathcal{Z}_{0}$ and $S=A_{1} \in \mathcal{Z}_{1}$.

SC2: Let $v, v^{\prime} \in \mathbb{P}$ such that $v+v^{\prime} \leq 1$. Then there are $p, p^{\prime}, q \in\{0,1, \ldots\}$ such that $v=\frac{p}{2^{q}}$ and $v^{\prime}=\frac{p^{\prime}}{2^{q}}$, and $p+p^{\prime} \leq 2^{q}$.

By construction, $A_{q}^{p} \in \mathcal{Z}_{v}, A_{q}^{p^{\prime}} \in \mathcal{Z}_{v^{\prime}}$, and $A_{q}^{p+p^{\prime}} \in \mathcal{Z}_{v+v^{\prime}}$. By Step 5, $S \backslash A_{q}^{p} \sim A_{q}^{2^{q}-p}$. Since $p+p^{\prime} \leq 2^{q}$, thus by monotonicity and Step 2, $S \backslash A_{q}^{p} \succsim A_{q}^{p^{\prime}}$. By Step 3, $\left.\succsim\right|_{S \backslash A_{q}^{p}}$ satisfies $2-A S$, so by the 1-AS Lemma,

$$
\mathcal{G}^{S \backslash A_{q}^{p}}\left(A_{q}^{p^{\prime}}\right) \sim A_{q}^{p^{\prime}} .
$$

Thus we have disjoint $A_{q}^{p} \in \mathcal{Z}_{v}$ and $\mathcal{G}^{S \backslash A_{q}^{p}}\left(A_{q}^{p^{\prime}}\right) \in \mathcal{Z}_{v^{\prime}}$.

Since $\succsim$ satisfies $1-A S$ and, by monotonicity, $S \succsim A_{q}^{p} \cup \mathcal{G}^{S \backslash A_{q}^{p}}\left(A_{q}^{p^{\prime}}\right)$, thus by the 1-AS Lemma, $\mathcal{G}^{S}\left(A_{q}^{p} \cup \mathcal{G}^{S \backslash A_{q}^{p}}\left(A_{q}^{p^{\prime}}\right)\right) \sim A_{q}^{p} \cup \mathcal{G}^{S \backslash A_{q}^{p}}\left(A_{q}^{p^{\prime}}\right)$. Thus by Step 7,

$$
\begin{aligned}
A_{q}^{p+p^{\prime}} & =A_{q}^{p} \biguplus A_{q}^{p^{\prime}} \\
& =\mathcal{G}^{S}\left(A_{q}^{p} \cup \mathcal{G}^{S \backslash A_{q}^{p}}\left(A_{q}^{p^{\prime}}\right)\right) \\
& \sim A_{q}^{p} \cup \mathcal{G}^{S \backslash A_{q}^{p}}\left(A_{q}^{p^{\prime}}\right),
\end{aligned}
$$

so $A_{q}^{p} \cup \mathcal{G}^{S \backslash A_{q}^{p}}\left(A_{q}^{p^{\prime}}\right) \in \mathcal{Z}_{v+v^{\prime}}$.

SC3: Let $\left(v_{i}^{+}\right) \in \mathbb{2}^{\mathbb{N}}$ be non-increasing and let $\left(v_{i}^{+}\right) \in \mathbb{2}^{\mathbb{N}}$ be non-decreasing such that $\lim v_{i}^{+}=\lim v_{i}^{-}$.

(i) for each $i \in \mathbb{N}, v_{i}+w_{i} \leq 1$ and $v_{i}^{\prime}+w_{i}^{\prime} \leq 1$, and

(ii) $\lim v_{i}=\lim v_{i}^{\prime}$ and $\lim w_{i}=\lim w_{i}^{\prime}$. 
For each $i \in \mathbb{N}$, define $A_{i} \equiv A_{v_{i}}$ and $A_{i}^{\prime} \equiv A_{v_{i}^{\prime}}$. Then for each $i \in \mathbb{N}, A_{i} \in \mathcal{Z}_{v_{i}}, A_{i}^{\prime} \in \mathcal{Z}_{v_{i}^{\prime}}$. By Step $9,\left(A_{i}\right)$ and $\left(A_{i}^{\prime}\right)$ are monotonic, so by the Convergence Lemma, $\left(A_{i}\right)$ and $\left(A_{i}^{\prime}\right)$ are convergent, so by Step 10, $\lim A_{i} \sim \lim A_{i}^{\prime}$.

SC4: Let $\left(v_{i}\right),\left(w_{i}\right) \in 2^{\mathbb{N}}$ be monotonic such that for each $i \in \mathbb{N}, v_{i}+w_{i} \leq 1$. For each $i \in \mathbb{N}$, define $A_{i} \equiv A_{v_{i}}$ and $B_{i} \equiv \mathcal{G}^{S \backslash A_{i}}\left(A_{w_{i}}\right)$.

By Step 5 , for each $i \in \mathbb{N}, S \backslash A_{i} \sim A_{1-v_{i}}$, so since $1-v_{i} \geq w_{i}$, by Step $9, S \backslash A_{v_{i}} \succsim A_{w_{i}}$. Thus by Step 3 and the 1-AS Lemma, for each $i \in \mathbb{N}, B_{i} \sim A_{w_{i}}$. Altogether, for each $i \in \mathbb{N}, A_{i}$ and $B_{i}$ are disjoint with $A_{i} \in \mathcal{Z}_{v_{i}}$ and $B_{i} \in \mathcal{Z}_{w_{i}}$.

By Step 9, $\left(A_{i}\right)$ and $\left(B_{i}\right)$ are monotonic, and by construction, for each $i \in \mathbb{N}, A_{i}$ and $B_{i}$ are disjoint; thus by the Convergence Lemma, $\left(\mathcal{G}_{1}^{S}\left(A_{i}, B_{i}\right)\right)$ and $\left(\mathcal{G}_{2}^{S}\left(A_{i}, B_{i}\right)\right)$ are convergent. By the Idempotence Lemma, these are, respectively $\left(A_{i}\right)$ and $\left(B_{i}\right)$.

- Theorem 1: If $(S, \mathcal{A}, \succsim)$ is a purely- $\sigma$-catalogued qualitative probability space satisfying (monotone) continuity and $3-A S$, then it has a unique representation $\mu \in \mathbb{M}^{\sigma}(\mathcal{A})$.

Proof: The result follows immediately from the Supercabinet Construction Lemma and the Supercabinet Blueprint Lemma.

\section{Appendix 6}

In this appendix, we prove the Carving Lemma and Theorem 2.

Theorem KK is an implication of the cardinal analogue of the 1-AS Lemma (Kakeya, 1914; Kakeya, 1915) and the cardinal analogue of the Greedy Removal Lemma (Komornik, 2015). It provides conditions for a sequence of numbers to have $k$ disjoint subsequences whose sums correspond to a given list $v_{1}, v_{2}, \ldots, v_{k}$ :

Definition: A sequence $\left(\mu_{i}\right) \in[0,1]^{\mathbb{N}}$ is $k$-Kakeya if

(i) $\left(\mu_{i}\right)$ is non-increasing,

(ii) $\lim \mu_{i}=0$, and

(iii) for each $i \in \mathbb{N}, \sum_{j>i} \mu_{j} \geq k \mu_{i}$.

- Theorem KK (Kakeya, 1914; Kakeya, 1915; Komornik, 2015): For each $k$ Kakeya $\left(\mu_{i}\right) \in[0,1]^{\mathbb{N}}$ and each $v_{1}, v_{2}, \ldots, v_{k} \in\left[0, \sum \mu_{i}\right]$ such that $v_{1}+v_{2}+\ldots+v_{k} \leq \sum \mu_{i}$, there are pairwise disjoint $A_{1}, A_{2}, \ldots, A_{k} \subseteq \mathbb{N}$ such that for each $i \in\{1,2, \ldots, k\}$,

$$
\sum_{A_{k}} \mu_{i}=v_{k}
$$

The Carving Lemma states that if a first event that contains no atoms is at least as likely as a second event, then the first contains a subevent that is as likely as the second.

- Carving Lemma: If $(S, \mathcal{A}, \succsim)$ is a catalogued qualitative probability space satisfying (monotone) continuity, then for each pair $A, B \in \mathcal{A}$ such that $A \in \mathcal{A}^{\circ}$ and $A \succsim B$, there is $B^{\prime} \subseteq A$ such that $B^{\prime} \in \mathcal{A}$ and $B^{\prime} \sim B$. 
Proof: Let $(S, \mathcal{A}, \succsim), A, B$ satisfy the hypotheses. If $A \sim B$, then define $B^{\prime} \equiv A$ and we are done; if $\emptyset \sim B$, then define $B^{\prime} \equiv \emptyset$ and we are done; thus assume $A \succ B \succ \emptyset$. By monotonicity, $S^{\circ} \succsim A \succ \emptyset$.

For each pair $C, C^{\prime} \in \mathcal{A}^{\circ}$, define $C \succsim^{\circ} C^{\prime}$ if and only if $C \succsim C^{\prime}$. Since $S^{\circ} \succ \emptyset$, it is straightforward to verify that $\left(S^{\circ}, \mathcal{A}^{\circ}, \succsim^{\circ}\right)$ is an atomless qualitative probability space satisfying monotone continuity, so by Theorem V2, it has a unique representation $\mu^{\circ} \in \mathbb{M}^{\sigma}\left(\mathcal{A}^{\circ}\right)$ that is infinitely-divisible.

Define $V^{+}, V^{-} \subseteq\left[0, \mu^{\circ}(A)\right]$ by:

$$
\begin{aligned}
& V^{+} \equiv\left\{v \in\left[0, \mu^{\circ}(A)\right] \mid C \in \mathcal{A}^{\circ} \text { and } \mu^{\circ}(C)=v \text { implies } C \succsim B\right\}, \text { and } \\
& V^{-} \equiv\left\{v \in\left[0, \mu^{\circ}(A)\right] \mid C \in \mathcal{A}^{\circ} \text { and } \mu^{\circ}(C)=v \text { implies } B \succsim C\right\} .
\end{aligned}
$$

Since $A \succ B \succ \emptyset$, thus $\mu^{\circ}(A) \in V^{+}$and $0 \in V^{-}$, so $\inf V^{+}$and $\sup V^{-}$are well-defined. Since $\mu^{\circ}$ is infinitely-divisible, $V^{+} \cup V^{-}=\left[0, \mu^{\circ}(A)\right]$. Moreover, since $\mu^{\circ}$ represents $\succsim^{\circ}$, for each pair $v, v^{\prime} \in[0,1]$ (i) $v \in V^{+}$and $v^{\prime}>v$ implies $v^{\prime} \in V^{+}$, and (ii) $v \in V^{-}$and $v^{\prime}<v$ implies $v^{\prime} \in V^{-}$. Altogether, $\inf V^{+}=\sup V^{-} ;$define $v^{*} \equiv \inf V^{+}=\sup V^{-}$. Since $\mu^{\circ}$ is infinitely-divisible, there is $B^{\prime} \in \mathcal{A}^{\circ}$ such that $\mu^{\circ}\left(B^{\prime}\right)=v^{*}$.

First, assume $v^{*} \neq \mu^{\circ}(A)$. Since $\mu^{\circ}$ is infinitely-divisible, there is $\left(A_{i}\right) \in\left(\mathcal{A}^{\circ}\right)^{\mathbb{N}}$ such that

(1) for each $i \in \mathbb{N}, \mu^{\circ}\left(A_{i}\right)=v^{*}+\left(\frac{1}{2}\right)^{i}\left(\mu^{\circ}(A)-v^{*}\right)$, and

(2) $A_{1} \supseteq A_{2} \supseteq \ldots$

By (2), there is $A_{\infty} \equiv \lim \left(A_{i}\right)$. For each $i \in \mathbb{N}, v_{i}>v^{*}$, so $v_{i} \in V^{+}$, so $A_{i} \succsim B$; thus by continuity, $A_{\infty} \succsim B$. Since $\mu^{\circ}$ is $\sigma$-additive, by (1) $\mu^{\circ}\left(A_{\infty}\right)=v^{*}$, so $B^{\prime} \sim A_{\infty}$. Altogether, $v^{*} \neq 0$ implies $B^{\prime} \succsim B$.

By a symmetric argument, $v^{*} \neq 0$ implies $B \succsim B^{\prime}$. We cannot have that $v^{*}=0$, else $\emptyset \sim B^{\prime} \succsim B$, contradicting $B \succ \emptyset$. Similarly, we cannot have that $v^{*}=\mu^{\circ}(A)$, else $B \succsim B^{\prime} \sim A$, contradicting $A \succ B$. Thus $v^{*} \in\left(0, \mu^{\circ}(A)\right)$, so $B^{\prime} \sim B$ as desired.

- Theorem 2: If $(S, \mathcal{A}, \succsim)$ is a finitely-catalogued qualitative probability space satisfying (monotone) continuity and $3-A S$, then it has a unique representation $\mu \in \mathbb{M}^{\sigma}(\mathcal{A})$.

Proof: If $S^{\oplus}=\emptyset$, then we are done by Theorem V2, so assume $S^{\oplus} \neq \emptyset$.

○ Step 1: Define $\mu^{\circ}$.

For each pair $A, B \subseteq S^{\circ}$, define $A \succsim^{\circ} B$ if and only if $A \succsim B$. Since $\succsim$ satisfies $3-A S$ and monotonicity, thus $S^{\circ} \succ \emptyset$, so $S^{\circ} \succ^{\circ} \emptyset$. It is immediate that $\left(S^{\circ}, \mathcal{A}^{\circ}, \succsim^{\circ}\right)$ is an atomless qualitative probability space satisfying monotone continuity, so by Theorem V2, it has a unique representation $\mu^{\circ} \in \mathbb{M}^{\sigma}\left(\mathcal{A}^{\circ}\right)$, and $\mu^{\circ}$ is infinitely-divisible.

○ Step 2: Construct $\left(A_{i}^{\prime}\right) \in \mathcal{A}^{\mathbb{N}}$ partitioning $S$, and the $\sigma$-algebra $\mathcal{A}^{\prime} \subseteq \mathcal{A}$.

To define $\left(A_{i}^{\prime}\right) \in \mathcal{A}^{\mathbb{N}}$, we first define $\left(A_{i}\right) \in \mathcal{A}^{\mathbb{N}}$. For each $i \in\{1,2, \ldots,|I|\}$, define $A_{i} \equiv$ $\{i\}$. Since $S \backslash\left(\cup_{j \leq|I|} A_{j}\right)=S^{\circ}$, thus by 3 - $A S$ and separability, $S \backslash\left(\cup_{j \leq|I|} A_{j}\right) \succ A_{|I|} \succ \emptyset$. Clearly, $S \backslash\left(\cup_{j \leq|I|} A_{j}\right) \in \mathcal{A}^{\circ}$.

Let $A_{|I|+1} \subseteq S^{\circ}$ such that $S^{\circ} \succ A_{|I|} \succ A_{|I|+1} \succ \emptyset$; we can do this by the Carving Lemma and infinite-divisibility of $\mu^{\circ}$. By the Complement Lemma, $S \backslash\left(\cup_{j \leq|I|+1} A_{j}\right)=$ $S^{\circ} \backslash A_{|I|+1} \succ \emptyset$, and clearly, $S \backslash\left(\cup_{j \leq|I|+1} A_{j}\right) \in \mathcal{A}^{\circ}$.

For the recursive step, assume we have defined $A_{1}, A_{2}, \ldots, A_{i} \in \mathcal{A}$ such that 
(i) $\left(S \backslash \cup_{j \leq i} A_{j}\right) \succ \emptyset$, and

(ii) $\left(S \backslash \cup_{j \leq i} A_{j}\right) \in \mathcal{A}^{\circ}$.

Since $\left(S \backslash \cup_{j \leq i} A_{j}\right) \subseteq S^{\circ}$ and $\mu^{\circ}$ is infinitely-divisible, thus $\left(S \backslash \cup_{j \leq i} A_{i}\right)$ can be partitioned into $B_{1}, B_{2}, B_{3}, B_{4}$ that are equally-likely, according to both $\succsim^{\circ}$ and $\succsim$.

If $A_{i} \succsim B_{1}$, then define $A_{i+1} \equiv B_{1}$. Then $\left(S \backslash \cup_{j \leq i+1} A_{j}\right)=B_{2} \cup B_{3} \cup B_{4}$, which by separability and monotonicity is not null. Clearly, $\left(S \backslash \cup_{j \leq i} A_{j}\right) \in \mathcal{A}^{\circ}$.

If $B_{1} \succ A_{i}$, then by the Carving Lemma, there is $C \subseteq B_{1}$ such that $C \sim^{\circ} A_{i}$, and thus $C \sim A_{i}$. Then $\left(S \backslash \cup_{j \leq i+1} A_{j}\right)$ contains $B_{2} \cup B_{3} \cup B_{4}$, and thus by separability and monotonicity is not null.

Consider $\left(A_{j}\right)_{j>|I|}$, which by construction are pairwise-disjoint. We cannot have that $A_{|I|+1} \sim A_{|I|+2} \sim \ldots$, else $\mu^{\circ}\left(A_{|I|+1}\right)=\mu^{\circ}\left(A_{|I|+2}\right)=\ldots$, so $\mu^{\circ}\left(\cup_{j>|I|} A_{j}\right)>1$, contradicting that $\mu^{\circ} \in \mathbb{M}^{\sigma}\left(\mathcal{A}^{\circ}\right)$. Thus there is some smallest $i^{*} \in\{|I|+1,|I|+2, \ldots\}$ such that $A_{i^{*}} \succ A_{i^{*}+1}$. By construction, $\mu^{\circ}\left(A_{i^{*}+1}\right)=\frac{1}{4} \mu^{\circ}\left(S^{\circ} \backslash \cup_{j \leq i^{*}} A_{j}\right)$. Moreover, for each $i \geq i^{*}$ such that $\mu^{\circ}\left(A_{i+1}\right)=\frac{1}{4} \mu^{\circ}\left(S^{\circ} \backslash \cup_{j \leq i} A_{j}\right)$, by construction $A_{i+1} \succ A_{i+2}$ and $\mu^{\circ}\left(A_{i+2}\right)=$ $\frac{1}{4} \mu^{\circ}\left(S^{\circ} \backslash \cup_{j \leq i} A_{j}\right)$. Altogether, there is $i^{*} \in\{|I|+1,|I|+2, \ldots\}$ such that for each $i \geq i^{*}$, $\mu^{\circ}\left(A_{i+1}\right)=\frac{1}{4} \mu^{\circ}\left(S^{\circ} \backslash \cup_{j \leq i} A_{j}\right)$. It follows from the additivity of $\mu^{\circ}$ that

$$
\begin{aligned}
\sum_{j>i^{*}} \mu^{\circ}\left(A_{j}\right) & =\frac{1}{4} \mu^{\circ}\left(S^{\circ} \backslash \cup_{j \leq i^{*}} A_{j}\right)+\frac{1}{4} \mu^{\circ}\left(S^{\circ} \backslash \cup_{j \leq i^{*}+1} A_{j}\right)+\frac{1}{4} \mu^{\circ}\left(S^{\circ} \backslash \cup_{j \leq i^{*}+2} A_{j}\right)+\ldots \\
& =\frac{1}{4} \mu^{\circ}\left(S^{\circ} \backslash \cup_{j \leq i^{*}} A_{j}\right)+\frac{3}{4} \frac{1}{4} \mu^{\circ}\left(S^{\circ} \backslash \cup_{j \leq i^{*}} A_{j}\right)+\left(\frac{3}{4}\right)^{2} \frac{1}{4} \mu^{\circ}\left(S^{\circ} \backslash \cup_{j \leq i^{*}} A_{j}\right)+\ldots \\
& =\frac{1}{4} \mu^{\circ}\left(S^{\circ} \backslash \cup_{j \leq i^{*}} A_{j}\right) \sum_{i=0}^{\infty}\left(\frac{3}{4}\right)^{k} \\
& =\mu^{\circ}\left(S^{\circ} \backslash \cup_{j \leq i^{*}} A_{j}\right)
\end{aligned}
$$

Since $j \leq|I|$ implies $A_{j} \cap S^{\circ}=\emptyset$, thus

$$
\sum_{j>i^{*}} \mu^{\circ}\left(A_{j}\right)=\mu^{\circ}\left(S^{\circ} \backslash \cup_{i^{*} \geq j>|I|} A_{j}\right)
$$

Moreover, since $\left(A_{i}\right)$ is pairwise-disjoint and since $j>|I|$ implies $A_{j} \subseteq S^{\circ}$, thus by additivity of $\mu^{\circ}$,

$$
\begin{aligned}
\mu^{\circ}\left(S^{\circ} \backslash \cup A_{j}\right) & =\mu^{\circ}\left(S^{\circ}\right)-\sum_{j>|I|} \mu^{\circ} \\
& =\mu^{\circ}\left(S^{\circ}\right)-\left[\sum_{i^{*} \geq j>|I|} \mu^{\circ}\left(A_{j}\right)+\sum_{j>i^{*}} \mu^{\circ}\left(A_{j}\right)\right] \\
& =\mu^{\circ}\left(S^{\circ}\right)-\left[\mu^{\circ}\left(\cup_{i^{*} \geq j>|I|}\right) A_{j}+\mu^{\circ}\left(S^{\circ} \backslash \cup_{i^{*} \geq j>|I|} A_{j}\right)\right] \\
& =\mu^{\circ}\left(S^{\circ}\right)-\mu^{\circ}\left(S^{\circ}\right)=1-1=0 .
\end{aligned}
$$

Since $\mu^{\circ}$ represents $\succsim^{\circ}$, thus $\left(S^{\circ} \backslash \cup A_{j}\right)$ is null, according to both $\succsim^{\circ}$ and $\succsim$.

Define $A_{1}^{\prime} \equiv A_{1} \cup\left(S^{\circ} \backslash \cup A_{j}\right)$, and for each $i \in \mathbb{N} \backslash\{1\}$, define $A_{i}^{\prime} \equiv A_{i}$. By separability, for each $i \in \mathbb{N}, A_{i}^{\prime} \sim A_{i}$. By construction, $\left(A_{i}^{\prime}\right)$ partitions $S$, with $\left(A_{i}^{\prime}\right)_{i \leq|I|}$ partitioning $S^{\oplus}$ and $\left(A_{i}^{\prime}\right)_{i>|I|}$ partitioning $S^{\circ}$.

Define $\mathcal{A}^{\prime} \subseteq \mathcal{A}$ to be the $\sigma$-algebra generated by $\left(A_{i}^{\prime}\right): A^{\prime} \in \mathcal{A}^{\prime}$ if and only if there is $J \subseteq \mathbb{N}$ such that $A^{\prime}=\cup_{i \in J} A_{i}^{\prime}$.

○ STEP 3: $\left(\mu^{\circ}\left(A_{i}^{\prime}\right)\right)_{i>|I|}$ is 3-Kakeya. 
Throughout this step, we use the index set $\{|I|+1,|I|+2, \ldots\}$, and we suppress it for convenience. By construction, $\left(A_{i}^{\prime}\right)$ is non-increasing in likelihood, so since $\mu^{\circ}$ represents $\succsim^{\circ}$, thus $\left(\mu^{\circ}\left(A_{i}^{\prime}\right)\right)$ is non-increasing. Moreover, $\left(\mu^{\circ}\left(A_{i}^{\prime}\right)\right)$ is bounded, so it is convergent. We cannot have $\lim \mu^{\circ}\left(A_{i}^{\prime}\right)>0$, else $\sum \mu^{\circ}\left(A_{i}^{\prime}\right)=\infty$, which since $\mu^{\circ}$ is $\sigma$-additive implies $\mu^{\circ}\left(S^{\circ}\right)=\mu^{\circ}\left(\cup A_{i}^{\prime}\right)=\sum \mu^{\circ}\left(A_{i}^{\prime}\right)=\infty$, contradicting $\mu^{\circ}\left(S^{\circ}\right)=1$. Thus $\lim \mu^{\circ}\left(A_{i}^{\prime}\right)=0$.

Let $i>|I|$. By construction, $\mu^{\circ}\left(A_{i}^{\prime}\right) \leq \frac{1}{4} \mu^{\circ}\left(S^{\circ} \backslash \cup_{j<i} A_{j}^{\prime}\right)$. Since $\left(A_{i}^{\prime}\right)$ partitions $S^{\circ}$, $\mu^{\circ}\left(S^{\circ} \backslash \cup_{j<i} A_{j}^{\prime}\right)=\mu^{\circ}\left(\cup_{j \geq i} A_{j}^{\prime}\right)$, so by $\sigma$-additivity of $\mu^{\circ}, \mu^{\circ}\left(A_{i}^{\prime}\right) \leq \frac{1}{4}\left(\mu^{\circ}\left(A_{i}^{\prime}\right)+\sum_{j>i} \mu^{\circ}\left(A_{j}^{\prime}\right)\right)$. Rearranging, $\sum_{j>i} \mu^{\circ}\left(A_{j}^{\prime}\right) \geq 3 \mu^{\circ}\left(A_{i}^{\prime}\right)$. Since $i>|I|$ was arbitrary, we are done.

○ SteP 4: Define $\left(S^{*}, \mathcal{A}^{*}, \succsim^{*}\right), \Psi^{*}: \mathcal{A}^{*} \rightarrow \mathcal{A}^{\prime}$, and $\Psi^{\prime}: \mathcal{A}^{\prime} \rightarrow \mathcal{A}^{*}$.

Define $S^{*} \equiv\{1,2, \ldots\}$ and define $\mathcal{A}^{*} \equiv 2^{S}$. Define $\Psi^{*}: \mathcal{A}^{*} \rightarrow \mathcal{A}^{\prime}$ as follows: for each $A^{*} \in \mathcal{A}^{*}$,

$$
\Psi^{*}\left(A^{*}\right)=\cup_{i \in A^{*}} A_{i}^{\prime}
$$

For each pair $A, B \in \mathcal{A}^{*}$, define

$$
A \succsim^{*} B \text { if and only if } \Psi^{*}(A) \succsim \Psi^{*}(B) .
$$

Clearly, $\Psi^{*}$ is a bijection that preserves disjoint unions: if $A, B \in \mathcal{A}^{*}$ are disjoint, then $\Psi^{*}(A), \Psi^{*}(B) \in \mathcal{A}^{\prime}$ are disjoint.

Let $\Psi^{\prime}: \mathcal{A}^{\prime} \rightarrow \mathcal{A}^{*}$ be the inverse of $\Psi^{*}$; clearly, $\Psi^{\prime}$ preserves disjoint unions.

○ STEP 5: Verify $\left(S^{*}, \mathcal{A}^{*}, \succsim^{*}\right)$ is a purely-catalogued qualitative probability space satisfying monotone continuity and $3-A S$.

We verify each property in sequence:

Order: To see that $\succsim^{*}$ is complete, let $A, B \in \mathcal{A}^{*}$. Since $\succsim$ is complete, $\Psi^{*}(A) \succsim$ $\Psi^{*}(B)$; thus $A \succsim^{*} B$ as desired.

To see that $\succsim^{*}$ is transitive, let $A, B, C \in \mathcal{A}^{*}$ such that $A \succsim^{*} B$ and $B \succsim^{*} C$. Then $\Psi^{*}(A) \succsim \Psi^{*}(B)$ and $\Psi^{*}(B) \succsim \Psi^{*}(C)$, so by transitivity of $\succsim, \Psi^{*}(A) \succsim \Psi^{*}(C)$; thus $A \succsim^{*} C$ as desired.

Separability: Let $A, B, C \in \mathcal{A}^{*}$ such that $A \cap C=B \cap C=\emptyset$. Since $\Psi^{*}$ preserves disjoint pairs, $\Psi^{*}(A) \cap \Psi^{*}(C)=\Psi^{*}(B) \cap \Psi^{*}(C)=\emptyset$. Since $\succsim$ is separable, $\Psi^{*}(A) \succsim \Psi^{*}(B)$ if and only if $\Psi^{*}(A) \cup \Psi^{*}(C) \succsim \Psi^{*}(B) \cup \Psi^{*}(C)$; thus $A \succsim^{*} B$ if and only if $A \cup C \succsim^{*} B \cup C$, as desired.

Monotonicity: Let $A, B \in \mathcal{A}$ with $A \subseteq B$. Then $\Psi^{*}(A) \subseteq \Psi^{*}(B)$. Since $\succsim$ is monotonic, $\Psi^{*}(B) \succsim \Psi^{*}(A)$; thus $B \succsim^{*} A$, as desired.

Nondegeneracy: Since $\left(A_{i}\right)$ partitions $S$, thus $\Psi^{*}\left(S^{*}\right)=S$, and clearly $\Psi^{*}(\emptyset)=\emptyset$. Since $\succsim$ is monotonic and nondegenerate, $S \succ \emptyset$, so $\Psi^{*}\left(S^{*}\right) \succ \Psi^{*}(\emptyset)$, so $S^{*} \succ^{*} \emptyset$, as desired.

Continuity: Let $A \in \mathcal{A}^{*}$ and let $\left(B_{i}\right) \in\left(\mathcal{A}^{*}\right)^{\mathbb{N}}$ be convergent. Define $B \equiv \lim B_{i}$. Clearly, $\left(\Psi^{*}\left(B_{i}\right)\right)$ is convergent with $\Psi^{*}(B)=\lim \Psi^{*}\left(B_{i}\right)$. 
If for each $i \in \mathbb{N}, B_{i} \succsim^{*} A$, then for each $i \in \mathbb{N}, \Psi^{*}\left(B_{i}\right) \succsim \Psi^{*}(A)$. Since $\succsim$ is continuous, $\Psi^{*}(B) \succsim \Psi^{*}(A)$; thus $B \succsim^{*} A$, as desired.

If for each $i \in \mathbb{N}, A \succsim^{*} B_{i}$, then by a similar argument $A \succsim^{*} B$, as desired.

3-AS: Let $A \in \mathcal{A}^{*}$ be an atom. By construction, separability, and monotonicity, there is $s^{*} \in S^{*}$ such that $A=\left\{s^{*}\right\}$. Then $\Psi^{*}\left(\left\{s^{*}\right\}\right)=A_{s^{*}}^{\prime}$.

CASE 1: $s^{*} \leq|I|$. Then $A_{s^{*}}^{\prime}$ is an atom. Since $\succsim$ satisfies $3-A S$, there are

(i) pairwise-disjoint $\mathcal{B} \subseteq\left(\downarrow A_{s^{*}}^{\prime}\right)$, and

(ii) $\mathcal{B}_{1}, \mathcal{B}_{2}, \mathcal{B}_{3} \subseteq \mathcal{B}^{\prime}$ partitioning $\mathcal{B}$,

such that for each $i \in\{1,2,3\},\left(\cup \mathcal{B}_{i}\right) \succsim \Psi^{*}(A)$.

For each $i \in\{1,2,3\}$, define $B_{i}^{\oplus} \equiv\left(\cup \mathcal{B}_{i}\right) \cap S^{\oplus}$, define $B_{i}^{\circ} \equiv\left(\cup \mathcal{B}_{i}\right) \cap S^{\circ}$, and define $v_{i} \equiv \mu^{\circ}\left(B_{i}\right)$. Since $\mu^{\circ}\left(B_{1} \cup B_{2} \cup B_{3}\right) \leq 1$ and $\mu^{\circ}$ is additive, thus $v_{1}+v_{2}+v_{3} \leq 1=$ $\mu^{\circ}\left(S^{\circ}\right)=\sum_{i>|I|} \mu^{\circ}\left(A_{i}^{\prime}\right)$. By Step 3 and Theorem KK, there are disjoint $J_{1}, J_{2}, J_{3} \subseteq \mathbb{N}$ such that for each $i \in\{1,2,3\}, \sum_{j \in J_{i}} \mu^{\circ}\left(A_{j}^{\prime}\right)=v_{i}$.

For each $i \in\{1,2,3\}$, define

$$
\begin{aligned}
\mathcal{B}_{i}^{\prime} & \equiv\left\{A_{j}^{\prime} \in \mathcal{A}^{\prime} \mid A_{j}^{\prime} \in \mathcal{B}_{i}^{\oplus} \text { or } j \in J_{i}\right\}, \\
\mathcal{B}^{\prime} & \equiv \cup \mathcal{B}_{i}^{\prime}, \\
\mathcal{B}_{i}^{*} & \equiv\left\{\Psi^{\prime}(B) \mid B \in \mathcal{B}_{i}^{\prime}\right\}, \text { and } \\
\mathcal{B}^{*} & \equiv \cup \mathcal{B}_{i}^{*} .
\end{aligned}
$$

By construction, $\mathcal{B}^{\prime}$ is pairwise-disjoint. Let $B \in \mathcal{B}^{\prime}$. If $B \in \cup \mathcal{B}_{i}^{\oplus}$, then $B \in\left(\downarrow A_{s^{*}}^{\prime}\right)$ by construction. If there is $j \in \cup J_{i}$ such that $B=A_{j}^{\prime}$, then $A_{s^{*}}^{\prime} \succsim A_{|I|}^{\prime} \succ A_{|I|+1}^{\prime} \succsim A_{j}^{\prime}=B$, so $B \in\left(\downarrow A_{s^{*}}^{\prime}\right)$. Altogether, $\mathcal{B}^{\prime} \subseteq\left(\downarrow A_{s^{*}}^{\prime}\right)$. Clearly, $\mathcal{B}_{1}^{\prime}, \mathcal{B}_{2}^{\prime}, \mathcal{B}_{3}^{\prime}$ partition $\mathcal{B}^{\prime}$.

Let $i \in\{1,2,3\}$, define $B_{i}^{\prime} \equiv \cup_{j \in J_{i}} A_{j}^{\prime}$. Since $\mu^{\circ}$ is $\sigma$-additive, $\mu^{\circ}\left(B_{i}^{\prime}\right)=v_{i}$, so $B_{i}^{\prime} \sim B_{i}$, so by the Domination Lemma twice, $\left(\cup \mathcal{B}_{i}^{\prime}\right)=B_{i}^{\oplus} \cup B_{i}^{\prime} \sim B_{i}^{\oplus} \cup B_{i}^{\circ}=\left(\cup \mathcal{B}_{i}\right)$; thus $\left(\cup \mathcal{B}_{i}^{\prime}\right) \succsim$ $A_{s^{*}}^{\prime}$.

Since $\Psi^{\prime}$ preserves disjoint pairs, thus (i) $\mathcal{B}^{*}$ is pairwise-disjoint, (ii) $\mathcal{B}^{*} \subseteq\left(\downarrow\left\{s^{*}\right\}\right)$ (for $\succsim^{*}$ ), (iii) $\mathcal{B}_{1}^{*}, \mathcal{B}_{2}^{*}, \mathcal{B}_{3}^{*}$ partition $\mathcal{B}^{*}$, and (iv) for each $i \in\{1,2,3\},\left(\cup \mathcal{B}_{i}^{*}\right) \succsim^{*} A$. Since $A$ was an arbitrary atom, we are done.

CASE 2: $s^{*}>|I|$. By Step 3, there is $s^{* *} \geq s^{*}$ such that $\mu^{\circ}\left(A_{s^{*}}\right)=\mu^{\circ}\left(A_{s^{* *}}^{\prime}\right)>$ $\mu^{\circ}\left(A_{s^{* *}+1}^{\prime}\right)$. By Step 3 and Theorem KK, there are disjoint $J_{1}, J_{2}, J_{3} \subseteq\left\{s^{* *}, s^{* *}+1, \ldots\right\}$ such that for each $i \in\{1,2,3\}, \sum_{j \in J_{i}} \mu^{\circ}\left(A_{j}^{\prime}\right)=\mu^{\circ}\left(A_{s^{*}}^{\prime}\right)$.

For each $i \in\{1,2,3\}$, define

$$
\begin{aligned}
\mathcal{B}_{i}^{\prime} & \equiv\left\{A_{j}^{\prime} \in \mathcal{A}^{\prime} \mid j \in J_{i}\right\}, \\
\mathcal{B}^{\prime} & \equiv \cup \mathcal{B}_{i}^{\prime}, \\
\mathcal{B}_{i}^{*} & \equiv\left\{\Psi^{\prime}(B) \mid B \in \mathcal{B}_{i}^{\prime}\right\}, \text { and } \\
\mathcal{B}^{*} & \equiv \cup \mathcal{B}_{i}^{*} .
\end{aligned}
$$

It is straightforward to verify that $\mathcal{B}^{*}, \mathcal{B}_{1}^{*}, \mathcal{B}_{2}^{*}, \mathcal{B}_{3}^{*}$ are as desired, using a simpler argument than that of the previous case. Since $A$ was an arbitrary atom, we are done.

\section{Purely-Catalogued: Trivial.}

○ Step 6: Conclude. 
By Step 4 and the Supercabinet Construction Lemma, $\left(S^{*}, \mathcal{A}^{*}, \succsim^{*}\right)$ has a supercabinet $\left\{\mathcal{Z}_{v}^{*}\right\}$. For each $v \in \mathcal{Q}$, let $Z_{v}^{*} \in \mathcal{Z}_{v}^{*}$, and define $\mathbb{Z}_{v}$ to be the equivalence class of events in $\mathcal{A}$ for $\cup_{i \in Z_{v}^{*}} A_{i}$. It is straightforward to verify $\left\{\mathcal{Z}_{v}\right\}$ is a supercabinet for $(S, \mathcal{A}, \succsim)$, so by the Supercabinet Blueprint Lemma we are done.

\section{Appendix 7}

In this appendix, we prove Theorem 3.

- Theorem 3: If $(S, \mathcal{A}, \succsim)$ is a catalogued qualitative probability space satisfying monotone continuity and unlikely cores, then it has a unique representation $\mu \in \mathbb{M}^{\sigma}(\mathcal{A})$.

Proof: For each pair $A, B \in \mathcal{A}^{\circ}$, define $A \succsim^{\circ} B$ if and only if $A \succsim B$. Similarly, for each pair $A, B \in \mathcal{A}^{\oplus}$, define $A \succsim^{\oplus} B$ if and only if $A \succsim B$.

○ STEP 1: Define $\mu^{\circ} \in \mathbb{M}^{\sigma}\left(\mathcal{A}^{\circ}\right), \mu^{\oplus}: \mathcal{A}^{\oplus} \rightarrow[0,1]$, and $\mu: \mathcal{A} \rightarrow[0,1]$.

Since $S^{\circ} \succ S^{\oplus}$, by monotonicity $S^{\circ} \succ \emptyset$. It is immediate that $\left(S^{\circ}, \mathcal{A}^{\circ}, \succsim^{\circ}\right)$ is an atomless qualitative probability space satisfying monotone continuity; thus by Theorem V2, it has unique representation $\mu^{\circ} \in \mathbb{M}^{\sigma}\left(\mathcal{A}^{\circ}\right)$, and moreover $\mu^{\circ}$ is infinitely-divisible. If $S^{\oplus}=\emptyset$, we are done, so assume $S^{\oplus} \neq \emptyset$.

To define $\mu^{\oplus}: \mathcal{A}^{\oplus} \rightarrow[0,1]$, let $A^{\oplus} \in \mathcal{A}^{\oplus}$. By monotonicity, $S^{\circ} \succsim S^{\oplus} \succsim A^{\oplus}$; thus by the Carving Lemma, there is $A^{\circ} \in \mathcal{A}^{\circ}$ such that $A^{\oplus} \sim A^{\circ}$. Define $\mu^{\oplus}\left(A^{\oplus}\right) \equiv \mu^{\circ}\left(A^{\circ}\right)$.

Define the constant for normalization $\kappa \equiv \mu^{\oplus}\left(S^{\oplus}\right)+\mu^{\circ}\left(S^{\circ}\right)$; then $\kappa>0$. For each $A \in \mathcal{A}$, define

$$
\mu(A) \equiv \frac{\mu^{\oplus}\left(A \cap S^{\oplus}\right)+\mu^{\circ}\left(A \cap S^{\circ}\right)}{\kappa} .
$$

○ SteP 2: For each disjoint pair $A^{\oplus}, B^{\oplus} \in \mathcal{A}^{\oplus}$, there are disjoint $A^{\circ}, B^{\circ} \in \mathcal{A}^{\circ}$ such that $A^{\oplus} \sim A^{\circ}$ and $B^{\oplus} \sim B^{\circ}$.

Let $A^{\oplus}, B^{\oplus} \in \mathcal{A}^{\oplus}$ be disjoint. By monotonicity, $S^{\circ} \succsim S^{\oplus} \succsim A^{\oplus}$; thus by the Carving Lemma, there is $A^{\circ} \in \mathcal{A}^{\circ}$ such that $A^{\oplus} \sim A^{\circ}$. By separability, $A^{\oplus} \cup B^{\oplus} \sim A^{\circ} \cup B^{\oplus}$.

Define $C^{\circ} \equiv S^{\circ} \backslash A^{\circ}$. By monotonicity and the above observation,

$$
\begin{aligned}
A^{\circ} \cup C^{\circ} & =S^{\circ} \\
& \succsim S^{\oplus} \\
& \succsim A^{\oplus} \cup B^{\oplus} \\
& \sim A^{\circ} \cup B^{\oplus}
\end{aligned}
$$

so by separability, $C^{\circ} \succsim B^{\oplus}$. Thus by the Carving Lemma, there is $B^{\circ} \subseteq C^{\circ}$ such that $B^{\circ} \in \mathcal{A}^{\circ}$ and $B^{\oplus} \sim B^{\circ}$. By construction, $A^{\circ}$ and $B^{\circ}$ are disjoint, as desired.

○ STEP 3: $\mu$ is additive. 
Let $A, B \in \mathcal{A}$ be disjoint. We wish to show $\mu(A \cup B)=\mu(A)+\mu(B)$. Define $A^{\oplus} \equiv A \cap S^{\oplus}, A^{\circ} \equiv A \cap S^{\circ}, B^{\oplus} \equiv B \cap S^{\oplus}$, and $B^{\circ} \equiv B \cap S^{\circ}$. By definition,

$$
\begin{aligned}
\mu(A \cup B) & =\frac{\mu^{\oplus}\left(A^{\oplus} \cup B^{\oplus}\right)+\mu^{\circ}\left(A^{\circ} \cup B^{\circ}\right)}{\kappa}, \\
\mu(A) & =\frac{\mu^{\oplus}\left(A^{\oplus}\right)+\mu^{\circ}\left(A^{\circ}\right)}{\kappa}, \text { and } \\
\mu(B) & =\frac{\mu^{\oplus}\left(B^{\oplus}\right)+\mu^{\circ}\left(B^{\circ}\right)}{\kappa} .
\end{aligned}
$$

Since $\mu^{\circ} \in \mathbb{M}^{\sigma}\left(\mathcal{A}^{\circ}\right)$, thus $\mu^{\circ}\left(A^{\circ} \cup B^{\circ}\right)=\mu^{\circ}\left(A^{\circ}\right)+\mu^{\circ}\left(B^{\circ}\right)$, so it suffices to show $\mu^{\oplus}\left(A^{\oplus} \cup\right.$ $\left.B^{\oplus}\right)=\mu^{\oplus}\left(A^{\oplus}\right)+\mu^{\oplus}\left(B^{\oplus}\right)$.

Since $A^{\oplus}$ and $B^{\oplus}$ are disjoint, by Step 2, there are disjoint $A^{\prime}, B^{\prime} \in \mathcal{A}^{\circ}$ such that $A^{\oplus} \sim A^{\prime}$ and $B^{\oplus} \sim B^{\prime}$. By the Domination Lemma twice, $A^{\oplus} \cup B^{\oplus} \sim A^{\prime} \cup B^{\prime}$. Thus by construction of $\mu^{\oplus}$,

$$
\begin{aligned}
\mu^{\oplus}\left(A^{\oplus} \cup B^{\oplus}\right) & =\mu^{\circ}\left(A^{\prime} \cup B^{\prime}\right), \\
\mu^{\oplus}\left(A^{\oplus}\right) & =\mu^{\circ}\left(A^{\prime}\right), \text { and } \\
\mu^{\oplus}\left(B^{\oplus}\right) & =\mu^{\circ}\left(B^{\prime}\right) .
\end{aligned}
$$

Since $\mu^{\circ} \in \mathbb{M}^{\sigma}\left(\mathcal{A}^{\circ}\right)$, thus $\mu^{\circ}\left(A^{\prime} \cup B^{\prime}\right)=\mu^{\circ}\left(A^{\prime}\right)+\mu^{\circ}\left(B^{\prime}\right)$, so $\mu^{\oplus}\left(A^{\oplus} \cup B^{\oplus}\right)=\mu\left(A^{\oplus}\right)+\mu\left(B^{\oplus}\right)$, as desired.

\section{- Step 4: Conclude.}

Let $A, B \in \mathcal{A}$. We wish to show $A \succsim B$ if and only if $\mu(A) \geq \mu(B)$. The idea of the proof is to construct, by algorithm, a list of pairs $(A, B)=\left(A_{1}, B_{1}\right),\left(A_{2}, B_{2}\right),\left(A_{3}, B_{3}\right), \ldots$ such that for each $i$,

(i) $A_{i} \succsim B_{i}$ if and only if $A_{i+1} \succsim B_{i+1}$, and

(ii) $\mu\left(A_{i}\right) \geq \mu\left(B_{i}\right)$ if and only if $\mu\left(A_{i+1}\right) \geq \mu\left(B_{i+1}\right)$.

Moreover, the process is guaranteed to terminate with some pair $\left(A_{\Omega}, B_{\Omega}\right)$ for which the statement " $A_{\Omega} \succsim B_{\Omega}$ if and only if $\mu\left(A_{\Omega}\right) \geq \mu\left(B_{\Omega}\right)$ " can be established, immediately proving " $A \succsim B$ if and only if $\mu(A) \geq \mu(B)$," as desired. Visually, it can be helpful to think of this list of pairs as a "logical ladder," where the rails and rungs are formed of if-and-only-if arrows. For convenience, given $A_{i}, B_{i}, A_{i+1}, B_{i+1}$, let us say "we can continue with $A_{i+1}$ and $B_{i+1}$ " as shorthand for statements (i) and (ii) above.

Given $A_{i}, B_{i} \in \mathcal{A}$, define the following four events:

$$
\begin{aligned}
A_{i}^{\circ} & \equiv A_{i} \cap S^{\circ}, \\
A_{i}^{\oplus} & \equiv A_{i} \cap S^{\oplus}, \\
B_{i}^{\circ} & \equiv B_{i} \cap S^{\circ}, \text { and } \\
B_{i}^{\oplus} & \equiv B_{i} \cap S^{\oplus} .
\end{aligned}
$$

Moreover, define $n_{i}^{\circ}, n_{i}^{\oplus} \in\{0,1,2\}$ by:

$$
\begin{aligned}
n_{i}^{\circ} & \equiv\left|\left\{C \in\left\{A_{i}^{\circ}, B_{i}^{\circ}\right\} \mid C \neq \emptyset\right\}\right|, \text { and } \\
n_{i}^{\oplus} & \equiv\left|\left\{C \in\left\{A_{i}^{\oplus}, B_{i}^{\oplus}\right\} \mid C \neq \emptyset\right\}\right| .
\end{aligned}
$$

Given $A_{i}, B_{i}$, the procedure is defined by following the earliest instruction that applies: 
1. If $A_{i}=\emptyset$ or $B_{i}=\emptyset$, then terminate.

In the former case, $\emptyset \succsim B_{i}$ implies (by monotonicity and the Domination Lemma) $\emptyset \succsim B_{i}^{\oplus}$ and $\emptyset \succsim B_{i}^{\circ}$; thus $0=\mu^{\oplus}(\emptyset) \geq \mu^{\oplus}\left(B_{i}^{\oplus}\right)$ and $0=\mu^{\circ}(\emptyset) \geq \mu^{\circ}\left(B_{i}^{\circ}\right)$, so by construction $0=\mu(\emptyset)=\mu\left(B_{i}\right)$. Conversely, $\mu(\emptyset) \geq \mu\left(B_{i}\right)$ implies $0 \geq \mu^{\oplus}\left(B_{i}^{\oplus}\right)$ and $0 \geq \mu^{\circ}\left(B_{i}^{\circ}\right)$; thus $\emptyset \succsim B_{i}^{\oplus}$ and $\emptyset \succsim B_{i}^{\circ}$, so by the Domination Lemma $\emptyset \succsim B_{i}$.

In the latter case, we have directly that $A_{i} \succsim \emptyset$ and $\mu\left(A_{i}\right) \geq \mu(\emptyset)$.

2. If $n_{i}^{\circ}=0$ or $n_{i}^{\oplus}=0$, then terminate; by construction of $\mu, A_{i} \succsim B_{i}$ if and only if $\mu\left(A_{i}\right) \geq \mu\left(B_{i}\right)$.

3. If $n_{i}^{\circ}=2$, then there are two cases.

- If $A_{i}^{\circ} \succsim B_{i}^{\circ}$, then by the Carving Lemma, there is $C^{\circ} \subseteq A_{i}^{\circ}$ such that $C^{\circ} \sim B_{i}^{\circ}$. Define $B_{i}^{\prime} \equiv\left(B_{i} \backslash B_{i}^{\circ}\right) \cup C^{\circ}$. By separability and additivity of $\mu$, we can continue with $A_{i}$ and $B_{i}^{\prime}$. Define $A_{i+1} \equiv A_{i} \backslash C^{\circ}$ and $B_{i+1} \equiv B_{i}^{\prime} \backslash C^{\circ}$. By separability and additivity of $\mu$, we can continue with $A_{i+1}$ and $B_{i+1}$.

- If $B_{i}^{\circ} \succsim A_{i}^{\circ}$, perform the operation analogous to that in the previous case.

In both cases, $n_{i+1}^{\circ}<n_{i}^{\circ}$ and $n_{i+1}^{\oplus}=n_{i}^{\oplus}$.

4. If $n_{i}^{\circ}=1$ and $n_{i}^{\oplus}=2$, then there are two cases.

- If $A_{i}^{\circ}=\emptyset$ and $A_{i}^{\oplus} \neq \emptyset$, then since by monotonicity $S^{\circ} \succsim S^{\oplus} \succsim A_{i}^{\oplus}$, by the Carving Lemma there is $A^{\circ} \in \mathcal{A}^{\circ}$ such that $A^{\circ} \sim A_{i}^{\oplus}$. Define $A_{i+1} \equiv A^{\circ}$ and define $B_{i+1} \equiv B_{i}$. By construction of $\mu$, we can continue with $A_{i+1}$ and $B_{i+1}$.

- If $B_{i}^{\circ}=\emptyset$ and $B_{i}^{\oplus} \neq \emptyset$, perform the operation analogous to that in the previous case.

In both cases, $n_{i}^{\circ}=2$ and $n_{i+1}^{\oplus}=1$.

5. If $n_{i}^{\circ}=1$ and $n_{i}^{\oplus}=1$, and neither $A_{i}$ nor $B_{i}$ is $\emptyset$, then there are two cases:

- If $A_{i}^{\circ}=\emptyset$ and $A_{i}^{\oplus} \neq \emptyset$, perform the operation analogous to that in the first case of the previous instruction.

- If $B_{i}^{\circ}=\emptyset$ and $B_{i}^{\oplus} \neq \emptyset$, perform the operation analogous to that in the second case of the previous instruction.

In both cases, $n_{i}^{\circ}=2$ and $n_{i+1}^{\oplus}=0$.

It is straightforward to verify that the process must terminate. By Theorem V1, since $\mu \in \mathbb{M}(\mathcal{A})$ represents $\succsim$, thus $\mu \in \mathbb{M}^{\sigma}(\mathcal{A})$. It is clear from its construction that $\mu$ is the unique such measure.

\section{Appendix 8}

In this appendix, we prove the Finite-Twin Lemma and Theorem 4.

- Finite-Twin Lemma: If $(S, \mathcal{A}, \succsim)$ is a $\sigma$-catalogued qualitative probability space satisfying (monotone) continuity and $1^{+}-A S$, then for each $s \in S^{\oplus}$ such that $S^{\circ} \succsim\{s+$ $1, s+2, \ldots\}$, there is $T_{s} \in \mathcal{A}$ such that

(i) $T_{s} \subseteq\{s+1, s+2, \ldots\} \cup S^{\circ}$, 
(ii) $T_{s} \cap S^{\oplus}$ is finite, and

(iii) $T_{s} \sim\{s\}$.

Proof: Let $(S, \mathcal{A}, \succsim)$ and $s \in S^{\oplus}$ satisfy the hypotheses. By $1^{+}-A S$, there is pairwisedisjoint $\mathcal{B} \subseteq(\downarrow\{s\})$ such that $(\cup \mathcal{B}) \succ\{s\}$. For each $B \in \mathcal{B}$ and each $s^{\prime} \in B \cap S^{\oplus}, s^{\prime}>s$; else since $B \in(\downarrow\{s\})$, by monotonicity $\{s\} \succ B \succsim\left\{s^{\prime}\right\} \succsim\{s\}$, contradicting $\{s\} \sim\{s\}$. Thus $(\cup \mathcal{B}) \subseteq\{s+1, s+2, \ldots\} \cup S^{\circ}$.

We claim there is $A \subseteq(\cup \mathcal{B})$ such that $A \cap S^{\oplus}$ is finite and $A \succ\{s\}$. Indeed, for each $i \in \mathbb{N}$, define $A_{i} \equiv(\cup \mathcal{B}) \cap\{1,2, \ldots, i\}$. We cannot have that for each $i \in \mathbb{N},\{s\} \succ A_{i}$, else by continuity, $\{s\} \succsim \lim A_{i}=(\cup \mathcal{B})$, contradicting $(\cup \mathcal{B}) \succ A_{i}$. Thus we can define $i^{*}$ as the least $i$ such that $A_{i} \succsim\{s\}$, and we can define $A \equiv A_{i^{*}}$. If $A \sim\{s\}$, then define $T_{s} \equiv A$ and we are done, so assume $A \succ\{s\}$.

If $A \cap S^{\oplus} \succ\{s\}$, then by monotonicity, $S^{\circ} \succsim\{s+1, s+2, \ldots\} \succsim A \cap S^{\oplus} \succsim\{s\}$, so by the Carving Lemma there is $T_{s} \subseteq S^{\circ}$ such that $T_{s} \sim\{s\}$ and we are done. If $\{s\} \succsim A \cap S^{\oplus}$, then we follow an argument analogous to that in the proof of the Carving Lemma. We begin by defining $V^{+}, V^{-} \subseteq[0,1]$ by:

$$
V^{+} \equiv\{v \in[0,1] \mid
$$

$$
\begin{aligned}
& V^{+} \equiv\left\{v \in\left[0, \mu^{\circ}\left(A \cap S^{\circ}\right)\right] \mid C \in \mathcal{A}^{\circ} \text { and } \mu^{\circ}(C)=v \text { implies }\left(A \cap S^{\oplus}\right) \cup C \succsim\{s\}\right\}, \text { and } \\
& V^{-} \equiv\left\{v \in\left[0, \mu^{\circ}\left(A \cap S^{\circ}\right)\right] \mid C \in \mathcal{A}^{\circ} \text { and } \mu^{\circ}(C)=v \text { implies }\{s\} \succsim\left(A \cap S^{\oplus}\right) \cup C\right\} .
\end{aligned}
$$

Through the analogous argument, there is $C \in \mathcal{A}^{\circ}$ such that $\left(A \cap S^{\oplus}\right) \cup C \sim\{s\}$; define $T_{s} \equiv\left(A \cap S^{\oplus}\right) \cup C$ and we are done.

- Theorem 4: If $(S, \mathcal{A}, \succsim)$ is a $\sigma$-catalogued qualitative probability space satisfying (monotone) continuity and $3^{+}-A S$, then it has a unique representation $\mu \in \mathbb{M}^{\sigma}(\mathcal{A})$.

Proof: Let $(S, \mathcal{A}, \succsim)$ be a catalogued qualitative probability space satisfying monotone continuity and $3^{+}-A S$. If $(S, \mathcal{A}, \succsim)$ is purely-catalogued, then we are done by Theorem 1 , and if $S^{\circ} \sim \emptyset$ the proof with Theorem 1 is trivial, so assume $S^{\circ} \succ \emptyset$.

○ STEP 1: Define ${ }^{\circ}$ and $\mu^{\circ}$.

For each pair $A, B \in \mathcal{A}^{\circ}$, define $A \succsim^{\circ} B$ if and only if $A \succsim B$. Since $S^{\circ} \succ \emptyset$, thus $\left(S^{\circ}, \mathcal{A}^{\circ}, \succsim^{\circ}\right)$ is an atomless qualitative probability space satisfying monotone continuity, so by Theorem V2, there is a unique $\mu^{\circ} \in \mathbb{M}^{\sigma}\left(\mathcal{A}^{\circ}\right)$ representing $\succsim^{\circ}$. Moreover, $\mu^{\circ}$ is infinitely-divisible.

○ Step 2: For each 'middle' $m \in S^{\oplus}$, define the space $\left(S^{\leftarrow m}, \mathcal{A}^{\leftarrow m}, \succsim^{\leftarrow m}\right)$, its associated $S^{\leftarrow m \oplus}$ and $\mathcal{A}^{\leftarrow m \oplus}$, the space $\left(S^{m \rightarrow}, \mathcal{A}^{m \rightarrow}, \succsim^{m \rightarrow}\right)$, and its associated $S^{m \rightarrow \oplus}$ and $\mathcal{A}^{m \rightarrow \oplus}$.

Let $m \in S^{\oplus}$.

Define $S^{\leftarrow m} \equiv\{1,2, \ldots, m\} \cup S^{\circ}$ and define $\mathcal{A}^{\leftarrow m} \equiv \mathcal{A} \cap 2^{S^{\leftarrow m}}$. For each pair $A, B \in$ $\mathcal{A}^{\leftarrow m}$, define $A \succsim \leftarrow m B$ if and only if $A \succsim B$. Define $S^{\leftarrow m \oplus} \equiv\{1,2, \ldots, m\}$ and define $\mathcal{A}^{\leftarrow m \oplus} \equiv 2^{S^{\leftarrow m \oplus}}$.

Define $S^{m \rightarrow} \equiv\{m, m+1, \ldots\} \cup S^{\circ}$ and define $\mathcal{A}^{m \rightarrow} \equiv \mathcal{A} \cap 2^{S^{m \rightarrow}}$. For each pair $A, B \in \mathcal{A}^{m \rightarrow}$, define $A \succsim^{m \rightarrow} B$ if and only if $A \succsim B$. Define $S^{m \rightarrow \oplus} \equiv\{m, m+1, \ldots\}$ and define $\mathcal{A}^{m \rightarrow \oplus} \equiv 2^{S^{m \rightarrow \oplus}}$. 
○ SteP 3: Identify $m^{\leftarrow *} \in S^{\oplus}$ such that for each $m \geq m^{\leftarrow *},\left(S^{\leftarrow m}, \mathcal{A}^{\leftarrow m}, \succsim^{\leftarrow m}\right)$ is a finitely-catalogued qualitative probability space satisfying monotone continuity and 3 $A S$.

It is straightforward to verify that for each $m \in S^{\oplus},\left(S^{\leftarrow m}, \mathcal{A}^{\leftarrow m}, \succsim^{\leftarrow m}\right)$ is a finitelycatalogued qualitative probability space satisfying monotone continuity.

We wish to identify $m^{\leftarrow *}$ and verify that it is as desired. To begin, for each $s \in S^{\oplus}$, let us say that $t \geq s$ is a truncation point of $s$ if there are

(i) pairwise-disjoint $\mathcal{B} \subseteq(\downarrow\{s\}) \subseteq \mathcal{A}^{\leftarrow t}$, and

(ii) $\mathcal{B}_{1}, \mathcal{B}_{2}, \mathcal{B}_{3} \subseteq \mathcal{B}$ partitioning $\mathcal{B}$,

such that for each $i \in\{1,2,3\},\left(\cup \mathcal{B}_{i}\right) t^{t}\{s\}$. Informally, if $t$ is a truncation point of $s$, then $\{s\}$ is third-order swarmed by less-likely non-null events even if we 'truncate' at $t$ by removing the tail of cores with higher index.

First, for each $s \in S^{\oplus}$, we construct a particular truncation point $\tau(s)$. Indeed, let $s \in S^{\oplus}$. Since $\succsim$ satisfies $3^{+}-A S$, there are

(i) pairwise-disjoint $\mathcal{B} \subseteq(\downarrow\{s\})$, and

(ii) $\mathcal{B}_{1}, \mathcal{B}_{2}, \mathcal{B}_{3} \subseteq \mathcal{B}^{\prime}$ partitioning $\mathcal{B}$,

such that for each $i \in\{1,2,3\},\left(\cup \mathcal{B}_{i}\right) \succ\{s\}$. Let $i \in\{1,2,3\}$. For each $j \in \mathbb{N}$, define $B_{j} \equiv\left(\cup \mathcal{B}_{i}\right) \cap S^{\leftarrow j}$. Then $\lim B_{j}=\left(\cup \mathcal{B}_{i}\right)$, and by separability, $\left(B_{j}\right)$ is increasing in likelihood. We cannot have that for each $j \in \mathbb{N},\{s\} \succ B_{j}$, else by continuity, $\{s\} \succsim\left(\cup \mathcal{B}_{i}\right)$, contradicting $\left(\cup \mathcal{B}_{i}\right) \succ\{s\}$. Thus we can define $j^{*}(i)$ as the least $j$ such that $B_{j} \succsim\{s\}$. Define

$$
\tau(s) \equiv \max \left\{s, j^{*}(1), j^{*}(2), j^{*}(3)\right\}
$$

define $\mathcal{B} \equiv \mathcal{B} \cap \mathcal{A}^{\leftarrow \tau(s)}$, and for each $i \in\{1,2,3\}$, define $\mathcal{B}_{i} \equiv \mathcal{B}_{i} \cap \mathcal{A}^{\leftarrow \tau(s)}$. It is straightforward to verify that these collections of events are as desired, so that $\tau(s) \geq s$ is indeed a truncation point of $s$.

Second, we claim there is $s^{*} \in S^{\oplus}$ such that for each $s \geq s^{*}, s$ is one of its own truncation points. Indeed, since $\mu^{\circ}$ is infinitely-divisible and represents $\succsim^{\circ}$, there are $A_{1}^{\circ}, A_{2}^{\circ}, A_{3}^{\circ}$ partitioning $S^{\circ}$ such that $A_{1}^{\circ} \sim A_{2}^{\circ} \sim A_{3}^{\circ}$. Since $S^{\circ} \succ \emptyset$, thus by separability, $A_{1}^{\circ} \succ \emptyset$. It cannot be that for each $s \in S^{\oplus},\{s\} \succ A_{1}^{\circ}$; else by continuity, $\emptyset=\lim \{s\} \succsim A_{1}^{\circ}$, contradicting $A_{1}^{\circ} \succ \emptyset$. Thus we can define $s^{*}$ to be the least $s \in S^{\oplus}$ such that $A_{1}^{\circ} \succsim\{s\}$. To see $s^{*}$ is as desired, let $s \geq s^{*}$. Then $A_{1}^{\circ} \succsim\left\{s^{*}\right\} \succsim\{s\}$, so by the Carving Lemma, there is $A_{s}^{\circ} \subseteq A_{1}^{\circ}$ such that $A_{s}^{\circ} \sim\{s\} \succ \emptyset$. Then $\mu^{\circ}\left(A_{s}^{\circ}\right)>0$. Since $\mu^{\circ}$ is infinitelydivisible, for each $i \in\{1,2,3\}$, there is $\mathcal{B}_{i}$ be partitioning $A_{i}^{\circ}$ such that for each $B \in \mathcal{B}_{i}$, $\mu^{\circ}\left(A_{s}^{\circ}\right)>\mu^{\circ}(B)>0$. Define $\mathcal{B}=\cup \mathcal{B}_{i}$. It is straightforward to verify that these collections of events are as desired, so that $s$ is indeed a truncation point of $s$.

Finally, define $m^{\leftarrow *} \equiv \max \left\{\tau(1), \tau(2), \ldots, \tau\left(s^{*}-1\right), s^{*}\right\}$. To see that $m^{\leftarrow *}$ is as desired, let $m \geq m^{\leftarrow *}$. Then the cores in $\left(S^{\leftarrow m}, \mathcal{A}^{\leftarrow m}, \succsim^{\leftarrow m}\right)$ are $\{1,2, \ldots, m\}$. By construction, for each $s \in\{1,2, \ldots, m\}$, there is a truncation point of $s$ in $\{1,2, \ldots, m\}$. Thus $\left(S^{\leftarrow m}, \mathcal{A}^{\leftarrow m}, \succsim \leftarrow m\right)$ satisfies 3 - $A S$, as desired.

○ SteP 4: Identify $m^{* \rightarrow} \in S^{\oplus}$ such that for each $m \geq m^{* \rightarrow},\left(S^{m \rightarrow}, \mathcal{A}^{m \rightarrow}, \succsim^{m \rightarrow}\right)$ is a catalogued probability space satisfying monotone continuity and unlikely cores.

It is straightforward to verify that for each $m \in S^{\oplus},\left(S^{m \rightarrow}, \mathcal{A}^{m \rightarrow}, \succsim^{m \rightarrow}\right)$ is a catalogued qualitative probability space satisfying monotone continuity. 
We wish to identify $m^{* \rightarrow}$ and verify that it is as desired. It cannot be that for each $s \in S^{\oplus},\{s, s+1, \ldots\} \succ S^{\circ}$; else by continuity, $\emptyset=\lim _{s \rightarrow \infty}\{s, s+1, \ldots\} \succsim S^{\circ}$, contradicting $S^{\circ} \succ \emptyset$. Let $m^{* \rightarrow}$ be the least $s \in S^{\oplus}$ such that $S^{\circ} \succsim\{s, s+1, \ldots\}$. To see that $m^{* \rightarrow}$ is as desired, let $m \geq m^{* \rightarrow}$. Then the cores in $\left(S^{m \rightarrow}, \mathcal{A}^{m \rightarrow}, \succsim^{m \rightarrow}\right)$ are $\{m, m+1, \ldots\}$. By monotonicity, $S^{\circ} \succsim\left\{m^{* \rightarrow}, m^{* \rightarrow}+1, \ldots\right\} \succsim\{m, m+1, \ldots\}$; thus $\left(S^{m \rightarrow}, \mathcal{A}^{m \rightarrow}, \succsim^{m \rightarrow}\right)$ satisfies unlikely cores, as desired.

- Step 5: Define $m^{*}$ such that for each $m \geq m^{*}$, there is a unique $\mu^{m} \in \mathbb{M}^{\sigma}(\mathcal{A})$ such that

(i) for each pair $A, B \in \mathcal{A}^{\leftarrow m}, A \succsim B$ if and only if $\mu^{m}(A) \geq \mu^{m}(B)$, and

(ii) for each pair $A, B \in \mathcal{A}^{m+1 \rightarrow}, A \succsim B$ if and only if $\mu^{m}(A) \geq \mu^{m}(B)$.

Define $m^{*} \equiv \max \left\{m^{\leftarrow *}, m^{* \rightarrow}\right\}$. Let $m \geq m^{*}$. To define $\mu^{m} \in \mathbb{M}^{\sigma}(\mathcal{A})$, we first define $\mu^{\leftarrow m} \in \mathbb{M}^{\sigma}\left(\mathcal{A}^{\leftarrow m}\right)$ and $\mu^{m+1 \rightarrow} \in \mathbb{M}^{\sigma}\left(\mathcal{A}^{m+1 \rightarrow}\right)$.

Since $m \geq m^{*} \geq m^{\leftarrow *}$, by Step $3,\left(S^{\leftarrow m}, \mathcal{A}^{\leftarrow m}, \succsim^{\leftarrow m}\right)$ is a finitely-catalogued qualitative probability space satisfying monotone continuity and $3-A S$. Thus by Theorem 2 , there is a unique $\mu^{\leftarrow m} \in \mathbb{M}^{\sigma}\left(\mathcal{A}^{\leftarrow m}\right)$ representing $\succsim^{\leftarrow m}$.

Since $m \geq m^{*} \geq m^{* \rightarrow},\left(S^{m+1 \rightarrow}, \mathcal{A}^{m+1 \rightarrow}, \succsim^{m+1 \rightarrow}\right)$ is a finitely-catalogued qualitative probability space satisfying monotone continuity and unlikely cores. Thus by Theorem 3 , there is a unique $\mu^{m+1 \rightarrow} \in \mathbb{M}^{\sigma}\left(\mathcal{A}^{m+1 \rightarrow}\right)$ representing $\succsim^{m+1 \rightarrow}$.

Assume we have $\mu^{m} \in \mathbb{M}^{\sigma}(\mathcal{A})$ satisfying the requirements. Then for each $A \in \mathcal{A}^{\leftarrow m}$, we can define $\mu^{\leftarrow}(A)$ by:

$$
\mu^{\leftarrow}(A) \equiv \frac{\mu^{m}(A)}{\mu^{m}\left(S^{\leftarrow m}\right)} .
$$

It is straightforward to verify that $\mu^{\leftarrow} \in \mathbb{M}^{\sigma}\left(\mathcal{A}^{\leftarrow m}\right)$ and $\mu^{\leftarrow}$ represents $\left(S^{\leftarrow m}, \mathcal{A}^{\leftarrow m}, \mathcal{A}^{\leftarrow m}\right)$; thus by the uniqueness of $\mu^{\leftarrow m}, \mu^{m}(A)=\mu^{\leftarrow m}(A) \mu^{m}\left(S^{\leftarrow m}\right)$. Since $\mu^{m}$ is additive, $\mu^{m}(A)=\mu^{\leftarrow m}(A)\left[\mu^{m}\left(S^{\circ}\right)+\mu^{m}\left(S^{\leftarrow m \oplus}\right)\right]$.

Similarly, for each $A \in \mathcal{A}^{m+1 \rightarrow}, \mu^{m}(A)=\mu^{m+1 \rightarrow}(A)\left[\mu^{m}\left(S^{\circ}\right)+\mu^{m}\left(S^{m+1 \rightarrow \oplus}\right)\right]$.

Altogether, for each $A \in\left(\mathcal{A}^{\leftarrow m} \cup \mathcal{A}^{m+1 \rightarrow}\right), \mu^{m}(A)$ can be written in terms of $\mu^{m}\left(S^{\circ}\right)$, $\mu^{m}\left(S^{\leftarrow m \oplus}\right)$, and $\mu^{m}\left(S^{m+1 \rightarrow \oplus}\right)$. For clarity, let us define the 'unknown' $x_{1}, x_{2}, x_{3}>0$ and the 'known' $a, b, c, d>0$ by:

$$
\begin{aligned}
x_{1} & \equiv \mu^{m}\left(S^{\circ}\right), \\
x_{2} & \equiv \mu^{m}\left(S^{\leftarrow m \oplus}\right), \\
x_{3} & \equiv \mu^{m}\left(S^{m+1 \rightarrow \oplus}\right), \\
a & \equiv \mu^{\leftarrow m}\left(S^{\circ}\right), \\
b & \equiv \mu^{m+1 \rightarrow}\left(S^{\circ}\right), \\
c & \equiv \mu^{\leftarrow m}\left(S^{\leftarrow m \oplus}\right), \text { and } \\
d & \equiv \mu^{m+1 \rightarrow}\left(S^{m+1 \rightarrow \oplus}\right) .
\end{aligned}
$$

Then we have the following system of equations:

$$
\begin{aligned}
x_{1} & =a\left(x_{1}+x_{2}\right), \\
x_{1} & =b\left(x_{1}+x_{3}\right), \\
x_{2} & =c\left(x_{1}+x_{2}\right), \\
x_{3} & =d\left(x_{1}+x_{3}\right), \text { and } \\
x_{1}+x_{2}+x_{3} & =1 .
\end{aligned}
$$


As each variable is positive, it is straightforward that this system has a unique solution.

To define $\mu$, first define $\mu^{m}\left(S^{\circ}\right), \mu^{m}\left(S^{\leftarrow m \oplus}\right)$, and $\mu^{m}\left(S^{m+1 \rightarrow \oplus}\right)$ using the solution to the above system. Then, for each $A \in\left(\mathcal{A}^{\leftarrow m} \cup \mathcal{A}^{m+1 \rightarrow}\right)$, define $\mu^{m}(A)$ using $\mu^{m}\left(S^{\circ}\right)$, $\mu^{m}\left(S^{\leftarrow m \oplus}\right)$, and $\mu^{m}\left(S^{m+1 \rightarrow \oplus}\right)$ as specified above. Finally, for each $A \in \mathcal{A}$, define

$$
\mu^{m}(A) \equiv \mu^{m}\left(A \cap S^{\circ}\right)+\mu^{m}\left(A \cap S^{\leftarrow m \oplus}\right)+\mu^{m}\left(A \cap S^{m+1 \rightarrow \oplus}\right) .
$$

By construction, $\mu^{m}(S)=1$. Moreover, the restriction of $\mu^{m}$ to $\mathcal{A}^{\leftarrow m}$ is a positive multiple of $\mu^{\leftarrow m}$, and the restriction of $\mu^{m}$ to $\mathcal{A}^{\rightarrow m}$ is a positive multiple of $\mu^{m+1 \rightarrow}$. Since $\mu^{\leftarrow m}$ is $\sigma$-additive on $\mathcal{A}^{\leftarrow m}$ and $\mu^{m+1 \rightarrow}$ is $\sigma$-additive on $\mathcal{A}^{m+1 \rightarrow}$, thus by construction, $\mu^{m}$ is $\sigma$-additive across $\mathcal{A}$. Since $\mu^{\leftarrow m}$ represents $\succsim^{\leftarrow m}$ and $\mu^{m+1 \rightarrow}$ represents $\succsim^{m+1 \rightarrow}$, altogether $\mu^{m}$ is as desired. It is clear from its construction that $\mu^{m}$ is the unique such measure.

○ SteP 6: For each pair $m, m^{\prime} \geq m^{*}, \mu^{m}=\mu^{m^{\prime}}$; define $\mu$ to be this measure.

We prove that for each $m \geq m^{*}, \mu^{m}=\mu^{m+1}$. Let $m \geq m^{*}$. By Step 5, to show $\mu^{m}=\mu^{m+1}$, it suffices to show

(i) for each pair $A, B \in \mathcal{A}^{\leftarrow m}, A \succsim B$ if and only if $\mu^{m+1}(A) \geq \mu^{m+1}(B)$, and

(ii) for each pair $A, B \in \mathcal{A}^{m+1 \rightarrow}, A \succsim B$ if and only if $\mu^{m+1}(A) \geq \mu^{m+1}(B)$,

since $\mu^{m}$ is the unique measure satisfying (i) and (ii). Moreover, (i) is immediate from the construction of $\mu^{m+1}$, as $\mathcal{A}^{\leftarrow m} \subseteq \mathcal{A}^{\leftarrow m+1}$. Thus it remains to show (ii).

By Step 4 and Step $5, S^{\circ} \succsim\{m+1\}$. Thus by the Carving Lemma, there is $A_{m+1}^{\circ} \in \mathcal{A}^{\circ}$ such that $A_{m+1}^{\circ} \sim\{m+1\}$. Since $A_{m+1}^{\circ},\{m+1\} \in \mathcal{A}^{m+1 \rightarrow}$, thus $\mu^{m}\left(A_{m+1}^{\circ}\right)=\mu^{m}(\{m+1\})$. Since $A_{m+1}^{\circ},\{m+1\} \in \mathcal{A}^{\leftarrow m+1}$, thus $\mu^{m+1}\left(A_{m+1}^{\circ}\right)=\mu^{m+1}(\{m+1\})$.

For each $A \in \mathcal{A}^{m+1 \rightarrow}$, define $v^{\prime}(A), v^{\prime \prime}(A)$ by:

$$
\begin{aligned}
v^{\prime}(A) & \equiv \frac{\mu^{m}(A)}{\mu^{m}\left(S^{m+2 \rightarrow}\right)}, \text { and } \\
v^{\prime \prime}(A) & \equiv \frac{\mu^{m+1}(A)}{\mu^{m+1}\left(S^{m+2 \rightarrow}\right)} .
\end{aligned}
$$

Let $\mu^{\prime}$ be the restriction of $v^{\prime}$ to $\mathcal{A}^{m+2 \rightarrow}$, and similarly let $\mu^{\prime \prime}$ be the restriction of $v^{\prime \prime}$ to $\mathcal{A}^{m+2 \rightarrow}$. Clearly, both $\mu^{\prime}$ and $\mu^{\prime \prime}$ are $\sigma$-measures representing $\left(S^{m+2 \rightarrow}, \mathcal{A}^{m+2 \rightarrow}, \succsim^{m+2 \rightarrow}\right)$, so by the uniqueness of $\mu^{m+2 \rightarrow}, \mu^{\prime}=\mu^{\prime \prime}$. Thus for each $A \in \mathcal{A}^{m+2 \rightarrow}, v^{\prime}(A)=v^{\prime \prime}(A)$.

Since $\mu^{m}\left(A_{m+1}^{\circ}\right)=\mu^{m}(\{m+1\})$ and $\mu^{m+1}\left(A_{m+1}^{\circ}\right)=\mu^{m+1}(\{m+1\})$, thus $v^{\prime}\left(A_{m+1}^{\circ}\right)=$ $v^{\prime}(\{m+1\})$ and $v^{\prime \prime}\left(A_{m+1}^{\circ}\right)=v^{\prime \prime}(\{m+1\})$, so

$$
\begin{aligned}
v^{\prime}(\{m+1\}) & =v^{\prime}\left(A_{m+1}^{\circ}\right) \\
& =v^{\prime \prime}\left(A_{m+1}^{\circ}\right) \\
& =v^{\prime \prime}(\{m+1\}) .
\end{aligned}
$$

Since $v^{\prime}$ and $v^{\prime \prime}$ are additive, thus $v^{\prime}=v^{\prime \prime}$. Since the restriction of $\mu^{m}$ to $\mathcal{A}^{m+1 \rightarrow}$ represents $\left(S^{m+1 \rightarrow}, \mathcal{A}^{m+1 \rightarrow}, \succsim^{m+1 \rightarrow}\right)$, and since $v^{\prime}$ is the product of this restriction with a positive number, altogether $v^{\prime}=v^{\prime \prime}$ represents $\left(S^{m+1 \rightarrow}, \mathcal{A}^{m+1 \rightarrow}, \succsim^{m+1 \rightarrow}\right)$. Since the restriction of $\mu^{m+1}$ to $\mathcal{A}^{m+1 \rightarrow}$ is the product of $v^{\prime \prime}$ with a positive number, altogether we are done.

- STEP 7: Define $S^{\leftarrow}$ and $S^{\rightarrow}$ such that $S^{\circ} \succsim S^{\rightarrow}$, then show that for the partition $\mathcal{S} \equiv\left\{S^{\circ}, S^{\leftarrow}, S^{\rightarrow}\right\}$, for each pair $S^{*}, S^{* *} \in \mathcal{S}$ and each pair $A, B \subseteq\left(S^{*} \cup S^{* *}\right), A \succsim B$ if and only if $\mu(A) \geq \mu(B)$. 
Define $S^{\leftarrow} \equiv S^{\leftarrow m^{*} \oplus}$, define $S^{\rightarrow} \equiv S^{m^{*}+1 \rightarrow \oplus}$, and define $\mathcal{S} \equiv\left\{S^{\circ}, S^{\leftarrow}, S^{\rightarrow}\right\}$. By Step 4 and Step $5, S^{\circ} \succsim S^{\rightarrow}$. Clearly, $\mathcal{S}$ is a partition. Let $S^{*}, S^{* *} \in \mathcal{S}$ and let $A, B \in \mathcal{A}$ with $A, B \subseteq\left(S^{*} \cup S^{* *}\right)$.

CASE 1: $S^{*}, S^{* *} \in\left\{S^{\circ}, S^{\leftarrow}\right\}$. Then we are done by Step 5 and Step 6 .

CASE 2: $S^{*}, S^{* *} \in\left\{S^{\circ}, S^{\rightarrow}\right\}$. Then we are done by Step 5 and Step 6 .

CAse 3: $\left\{S^{*}, S^{* *}\right\}=\left\{S^{\leftarrow}, S^{\rightarrow}\right\}$. Let $C \in \mathcal{A}$ with $C \subseteq S^{\leftarrow} \cup S^{\rightarrow}$, define $C^{\leftarrow} \equiv C \cap S^{\leftarrow}$, and define $C^{\rightarrow} \equiv C \cap S^{\rightarrow}$. By monotonicity, $S^{\circ} \succsim S^{\rightarrow} \succsim C^{\rightarrow}$, so by the Carving Lemma, there is $C^{\circ} \in \mathcal{A}^{\circ}$ such that $C^{\circ} \sim C^{\rightarrow}$. By Case 2, $\mu\left(C^{\circ}\right)=\mu\left(C^{\rightarrow}\right)$. Define $C^{\prime} \equiv C^{\leftarrow} \cup C^{\circ}$. By separability, $C^{\prime} \sim C$, and by additivity of $\mu, \mu\left(C^{\prime}\right)=\mu(C)$. Since $C$ was arbitrary, thus there are $A^{\prime}, B^{\prime} \in \mathcal{A}$ with $A^{\prime}, B^{\prime} \subseteq\left(S^{\leftarrow} \cup S^{\circ}\right)$ such that $A \sim A^{\prime}, B \sim B^{\prime}, \mu(A)=\mu\left(A^{\prime}\right)$, and $\mu(B)=\mu\left(B^{\prime}\right)$. By Case $1, A^{\prime} \succsim B^{\prime}$ if and only if $\mu\left(A^{\prime}\right) \geq \mu\left(B^{\prime}\right)$; thus $A \succsim B$ if and only if $\mu(A) \geq \mu(B)$, as desired.

- Step 8: Conclude.

A simpler version of the argument in this step is found in Step 4 of the proof of Theorem 3.

Let $A, B \in \mathcal{A}$. We wish to show $A \succsim B$ if and only if $\mu(A) \geq \mu(B)$. The idea of the proof is to construct, by algorithm, a list of pairs $(A, B)=\left(A_{1}, B_{1}\right),\left(A_{2}, B_{2}\right),\left(A_{3}, B_{3}\right), \ldots$ such that for each $i$,

(i) $A_{i} \succsim B_{i}$ if and only if $A_{i+1} \succsim B_{i+1}$, and

(ii) $\mu\left(A_{i}\right) \geq \mu\left(B_{i}\right)$ if and only if $\mu\left(A_{i+1}\right) \geq \mu\left(B_{i+1}\right)$.

Moreover, the process is guaranteed to terminate with some pair $\left(A_{\Omega}, B_{\Omega}\right)$ for which the statement " $A_{\Omega} \succsim B_{\Omega}$ if and only if $\mu\left(A_{\Omega}\right) \geq \mu\left(B_{\Omega}\right)$ " can be established, immediately proving " $A \succsim B$ if and only if $\mu(A) \geq \mu(B)$," as desired. Visually, it can be helpful to think of this list of pairs as a "logical ladder," where the rails and rungs are formed of if-and-only-if arrows. For convenience, given $A_{i}, B_{i}, A_{i+1}, B_{i+1}$, let us say "we can continue with $A_{i+1}$ and $B_{i+1}$ " as shorthand for statements (i) and (ii) above.

Given $A_{i}, B_{i} \in \mathcal{A}$, define the following six events:

$$
\begin{aligned}
A_{i}^{\circ} & \equiv A_{i} \cap S^{\circ}, \\
A_{i}^{\leftarrow} & \equiv A_{i} \cap S^{\leftarrow}, \\
A_{i}^{\rightarrow} & \equiv A_{i} \cap S^{\rightarrow}, \\
B_{i}^{\circ} & \equiv B_{i} \cap S^{\circ}, \\
B_{i}^{\leftarrow} & \equiv B_{i} \cap S^{\leftarrow}, \text { and } \\
B_{i}^{\rightarrow} & \equiv B_{i} \cap S^{\rightarrow} .
\end{aligned}
$$

Moreover, define $n_{i}^{\circ}, n_{i}^{\leftarrow}, n_{i} \in\{0,1,2\}$ by:

$$
\begin{aligned}
n_{i}^{\circ} & \equiv\left|\left\{C \in\left\{A_{i}^{\circ}, B_{i}^{\circ}\right\} \mid C \neq \emptyset\right\}\right|, \\
n_{i}^{\leftarrow} & \equiv\left|\left\{C \in\left\{A_{i}^{\leftarrow}, B_{i}^{\leftarrow}\right\} \mid C \neq \emptyset\right\}\right|, \text { and } \\
n_{i} & \equiv\left|\left\{C \in\left\{A_{i}^{\rightarrow}, B_{i}^{\rightarrow}\right\} \mid C \neq \emptyset\right\}\right| .
\end{aligned}
$$

Given $A_{i}, B_{i}$, the procedure is defined by following the earliest instruction that applies: 
1. If $A_{i}=\emptyset$ or $B_{i}=\emptyset$, then terminate.

In the former case, $\emptyset \succsim B_{i}$ implies (by monotonicity and the Domination Lemma) $\emptyset \succsim B_{i}^{\leftarrow}, \emptyset \succsim B_{i}^{\rightarrow}$, and $\emptyset \succsim B_{i}^{\circ}$; thus by Step 5 and Step $6,0=\mu(\emptyset) \geq \mu\left(B_{i}^{\leftarrow}\right)$ $0=\mu(\emptyset) \geq \mu\left(B_{i}\right)$, and $0=\mu(\emptyset) \geq \mu\left(B_{i}^{\circ}\right)$; so by additivity $0=\mu(\emptyset)=\mu\left(B_{i}\right)$. Conversely, $\mu(\emptyset) \geq \mu\left(B_{i}\right)$ implies $0 \geq \mu\left(B_{i}^{\leftarrow}\right), 0 \geq \mu\left(B_{i}^{\rightarrow}\right)$, and $0 \geq \mu\left(B_{i}^{\circ}\right)$; thus by Step 5 and Step $6, \emptyset \succsim B_{i}^{\leftarrow}, \emptyset \succsim B_{i}^{\rightarrow}$, and $\emptyset \succsim B_{i}^{\circ}$; so by the Domination Lemma $\emptyset \succsim B_{i}$.

In the latter case, we have directly that $A_{i} \succsim \emptyset$ and $\mu\left(A_{i}\right) \geq \mu(\emptyset)$.

2. If $n_{i}^{\circ}=0$, or $n_{i}^{\leftarrow}=0$, or $n_{i}=0$, then terminate; by Step 7, $A_{i} \succsim B_{i}$ if and only if $\mu\left(A_{i}\right) \geq \mu\left(B_{i}\right)$.

3. If $n_{i}^{\circ}=2$, then there are two cases.

- If $A_{i}^{\circ} \succsim B_{i}^{\circ}$, then by the Carving Lemma, there is $C^{\circ} \subseteq A_{i}^{\circ}$ such that $C^{\circ} \sim B_{i}^{\circ}$. Define $B_{i}^{\prime} \equiv\left(B_{i} \backslash B_{i}^{\circ}\right) \cup C^{\circ}$. By separability and additivity of $\mu$, we can continue with $A_{i}$ and $B_{i}^{\prime}$. Define $A_{i+1} \equiv A_{i} \backslash C^{\circ}$ and $B_{i+1} \equiv B_{i}^{\prime} \backslash C^{\circ}$. By separability and additivity of $\mu$, we can continue with $A_{i+1}$ and $B_{i+1}$.

- If $B_{i}^{\circ} \succsim A_{i}^{\circ}$, perform the operation analogous to that in the previous case.

In both cases, $n_{i+1}^{\circ}<n_{i}^{\circ}$ and $n_{i+1}=n_{i}$.

4. If $n_{i}^{\circ}=1$ and $n_{i}=2$, then there are two cases.

- If $A_{i}^{\circ}=\emptyset$ and $A_{i} \neq \emptyset$, then since by monotonicity $S^{\circ} \succsim S^{\rightarrow} \succsim A_{i}$, by the Carving Lemma there is $A^{\circ} \in \mathcal{A}^{\circ}$ such that $A^{\circ} \sim A_{i}$. Define $A_{i+1} \equiv\left(A_{i} \backslash A_{i}\right) \cup A^{\circ}$ and define $B_{i+1} \equiv B_{i}$. By separability and additivity of $\mu$, we can continue with $A_{i+1}$ and $B_{i+1}$.

- If $B_{i}^{\circ}=\emptyset$ and $B_{i} \rightarrow \emptyset$, perform the operation analogous to that in the previous case.

In both cases, $n_{i+1}=1$.

5. If $n_{i}^{\circ}=1, n_{i}=1$, and neither $A$ nor $B$ is contained in $S^{\leftarrow}$, then there are two cases:

- If $A_{i}^{\circ}=\emptyset$ and $A_{i} \neq \emptyset$, perform the operation analogous to that in the first case of the previous instruction.

- If $B_{i}^{\circ}=\emptyset$ and $B_{i} \neq \emptyset$, perform the operation analogous to that in the second case of the previous instruction.

In both cases, $\overrightarrow{n_{i+1}}=0$.

6. If $n_{i}^{\circ}=1, n_{i} \rightarrow=1$, either $A$ or $B$ is contained in $S^{\leftarrow}$, and neither $A_{i}$ nor $B_{i}$ is $\emptyset$, then there are two cases:

- If $A_{i}=A_{i}^{\leftarrow}$, then define $s \equiv \max A_{i}$. By the Finite-Twin Lemma, there is finite $T_{s} \subseteq\{s+1, s+2, \ldots\} \cup S^{\circ}$ such that $T_{s} \sim\{s\}$. Define $m^{* *} \equiv \max \left\{\max T_{s}, m^{*}\right\}$. Since $T_{s},\{s\} \in \mathcal{A}^{\leftarrow m^{* *}}$, thus by Step 5 and Step 6, $\mu\left(T_{s}\right)=\mu(\{s\})$. Define $A_{i+1} \equiv$ $\left(A_{i} \backslash\{s\}\right) \cup T_{s}$ and define $B_{i+1} \equiv B_{i}$. By separability and additivity of $\mu$, we can continue with $A_{i+1}$ and $B_{i+1}$.

- If $B_{i}=B_{i}^{\leftarrow}$, perform the operation analogous to that in the previous case. 
It is straightforward to verify that the process cannot indefinitely loop through the first four instructions. If the fifth instruction is ever reached, then the process terminates on the next step.

For each pair $A, B \subseteq S^{\leftarrow}$, let the statement $A$ is lexicographically greater than $B$, written $A>_{L E X} B$, be defined by:

$$
A>_{L E X} B \text { if and only if } \min A \backslash B<\min B \backslash A \text {. }
$$

Observe that each of the first five instructions sets $A_{i+1}^{\overleftarrow{ }} \equiv A_{i}^{\leftarrow}$ and $B_{i+1}^{\overleftarrow{ }} \equiv B_{i}^{\leftarrow}$. Moreover, each time the sixth instruction is reached, either $A_{i+1}^{\leftarrow}<_{L E X} A_{i}^{\leftarrow}$ or $B_{i+1}^{\leftarrow}<_{L E X} B_{i}^{\leftarrow}$ Because $\mathcal{A}^{\leftarrow}$ is finite, there is some $k$ for which $A_{k}^{\leftarrow}=\emptyset$ and there is some $k^{\prime}$ for which $B_{k^{\prime}}^{\overleftarrow{ }}=\emptyset$. Define $\Omega=\max \left\{k, k^{\prime}\right\}$, and the process terminates with $A_{\Omega}, B_{\Omega}$ at the latest.

Altogether, $\mu \in \mathbb{M}^{\sigma}(\mathcal{A})$ represents $(S, \mathcal{A}, \succsim)$. By Step 5 and Step 6, $\mu$ is the unique such measure.

\section{Appendix 9}

In this appendix, we prove Theorem 5 .

- Theorem 5: A triple $(S, \mathcal{A}, \succsim)$ is a qualitative probability space satisfying (monotone) continuity and $3^{+}-A S$ if and only if it has a unique representation $\mu \in \mathbb{M}^{\sigma}(\mathcal{A})$ such that for each $A \in \mathcal{A}$,

(i) if $A$ is an atom, $\mu(\cup(\downarrow A))>3 \mu(A)>0$, and

(ii) if $A$ contains no atoms, for each $\lambda \in[0,1]$, there is $B \subseteq A$ such that $\mu(B)=\lambda \mu(A)$.

Proof: It is trivial that our axioms are necessary for such a representation, so let $(S, \mathcal{A}, \succsim)$ be a qualitative probability space satisfying (monotone) continuity and $3^{+}-A S$.

- SteP 1: There is no increasing sequence of atoms.

Assume, by way of contradiction, there is $\left(A_{i}\right) \in\left(\mathcal{A}^{\alpha}\right)^{\mathbb{N}}$ such that $A_{1} \prec A_{2} \prec \ldots$ Then for each pair $i, j \in \mathbb{N}$ with $i>j$, by monotonicity, $A_{i} \succ A_{j} \succsim A_{i} \cap A_{j}$, so since $A_{i}$ is an atom, thus $A_{i} \cap A_{j} \sim \emptyset$. For each $i \in \mathbb{N}$, define $B_{i} \equiv A_{i} \backslash\left(\bigcup_{j<i} A_{j}\right)=A_{i} \backslash\left(\bigcup_{j<i} A_{j} \cap A_{i}\right)$; by monotonicity and separability, $\left(\bigcup_{j<i} A_{i} \cap A_{j}\right) \sim \emptyset$, so by separability $B_{i} \sim A_{i}$. By construction, the $B_{i}$ are pairwise-disjoint. But then for each $i \in \mathbb{N}, B_{i} \sim A_{i} \succsim A_{1}$, so by continuity, $\emptyset=\lim B_{i} \succsim A_{1}$, contradicting that $A_{1}$ is an atom.

- SteP 2: Construct index set $I$ and pairwise-disjoint atoms $\left(A_{i}\right)_{i \in I}$, indexed in nonincreasing order of likelihood, such that $S^{\circ} \equiv S \backslash\left(\cup A_{i}\right)$ contains no atoms.

If there are no atoms, define $I \equiv \emptyset$ and we are done; otherwise, we proceed inductively. For the base step, by Step 1 , there is a $\succsim$-maximum $A_{1}$ in $\mathcal{A}^{\alpha}$, and for each $B \in \mathcal{A}^{\alpha}$ such that $B \subseteq S \backslash A_{1}, A_{1} \succsim B$. For the inductive step, assume that $k \in \mathbb{N}$ and we have pairwise-disjoint atoms $A_{1}, A_{2}, \ldots, A_{k}$ such that

(1) $A_{1} \succsim A_{2} \succsim \ldots \succsim A_{k}$, and

(2) for each $\widetilde{\widetilde{B}} \in \widetilde{\mathcal{A}}^{\alpha}$ such that $B \subseteq S \backslash \bigcup_{i=1}^{k} A_{i}, A_{k} \succsim B$. 
Define $\mathcal{A}_{k}^{\alpha} \subseteq \mathcal{A}^{\alpha}$ by:

$$
\mathcal{A}_{k}^{\alpha} \equiv\left\{B \in \mathcal{A}^{\alpha} \mid B \subseteq S \backslash \bigcup_{i=1}^{k} A_{i}\right\} .
$$

If $\mathcal{A}_{k}^{\alpha}$ is empty, then we are done. If $\mathcal{A}_{k}^{\alpha}$ is nonempty, then by Step 1 it has $\succsim$-maximum $A_{k+1}$. Since $A_{k+1} \in \mathcal{A}^{\alpha}$ and $A_{k+1} \subseteq S \backslash \bigcup_{i=1}^{k} A_{i}$, thus by the inductive hypothesis, $A_{1}, A_{2}, \ldots, A_{k+1}$ are pairwise-disjoint atoms and $A_{1} \succsim A_{2} \succsim \ldots \succsim A_{k+1}$. By construction, for each $B \in \mathcal{A}^{\alpha}$ such that $B \subseteq S \backslash \bigcup_{i=1}^{k+1} A_{i}, A_{k+1} \succsim B$.

Define $S^{\circ} \equiv S \backslash\left(\cup A_{i}\right)$. If construction terminates at some step, then $I=\{1,2, \ldots,|I|\}$; otherwise $I=\mathbb{N}$. If $I$ is empty or finite, then $S^{\circ}$ contains no atoms by construction. If $I=\mathbb{N}$, then assume, by way of contradiction, there is an atom $A \subseteq S^{\circ}$. By construction, for each $i \in \mathbb{N}, A_{i} \succsim A$. But then by continuity, $\emptyset=\lim A_{i} \succsim A$, contradicting that $A$ is an atom.

○ STEP 3: Construct $\left(S^{*}, \mathcal{A}^{*}, \succsim^{*}\right)$ and the mappings $\Psi, \Psi^{\prime}, \Psi^{*}$.

Informally, to construct the catalogued space, we replace each $A_{i}$ with $\{i\}$. Formally, define $S^{*} \equiv S^{\circ} \cup I$. Let $\mathcal{A}^{*}$ be the $\sigma$-algebra generated by $\left(2^{S^{\circ}} \cap \mathcal{A}\right) \cup 2^{I}$; that is, $A \in \mathcal{A}^{*}$ if and only if there are $B_{1} \subseteq S^{\circ}$ and $B_{2} \subseteq I$ such that (i) $B_{1} \in \mathcal{A}$, and (ii) $A=B_{1} \cup B_{2}$.

Let $\mathcal{A}^{\prime} \subseteq \mathcal{A}$ be the collection of events that contain any intersected $A_{i}$ :

$$
\mathcal{A}^{\prime} \equiv\left\{A \in \mathcal{A} \mid \text { for each } i \in I, A \cap A_{i} \in\left\{\emptyset, A_{i}\right\}\right\}
$$

Define $\Psi: \mathcal{A} \rightarrow \mathcal{A}^{\prime}, \Psi^{\prime}: \mathcal{A}^{\prime} \rightarrow \mathcal{A}^{*}$, and $\Psi^{*}: \mathcal{A}^{*} \rightarrow \mathcal{A}^{\prime}$ by:

$$
\begin{aligned}
\Psi(A) & \equiv\left(A \cap S^{\circ}\right) \cup\left(\bigcup\left\{A_{i} \in \mathcal{A} \mid A \cap A_{i} \sim A_{i}\right\}\right) . \\
\Psi^{\prime}(A) & \equiv\left(A \cap S^{\circ}\right) \cup\left\{i \in I \mid A \cap A_{i}=A_{i}\right\} \\
\Psi^{*}(A) & \equiv \Psi^{\prime-1}(A) .
\end{aligned}
$$

Define $\succsim^{*}$ by: for each pair $A, B \in \mathcal{A}^{*}, A \succsim^{*} B$ if and only if $\Psi^{*}(A) \succsim \Psi^{*}(B)$.

○ SteP 4: $\Psi$ preserves likelihood.

We wish to prove that for each $A \in \mathcal{A}, A \sim \Psi(A)$. Indeed, define $B_{0} \equiv A \cap S^{\circ}$ and $C_{0} \equiv \Psi(A) \cap S^{\circ}$, and define $\left(B_{i}\right),\left(C_{i}\right) \in \mathcal{A}^{I}$ recursively by:

$$
\begin{aligned}
& B_{i+1} \equiv B_{i} \cup\left(A \cap A_{i+1}\right) \\
& C_{i+1} \equiv C_{i} \cup\left(\Psi(A) \cap A_{i+1}\right) .
\end{aligned}
$$

We claim that for each $i \in I, B_{i} \sim C_{i}$, which we prove by induction. For the base step, $B_{0}=C_{0}$. For the inductive step, assume that $B_{i} \sim C_{i}$. By definition of $\Psi$, $\left(A \cap A_{i+1}\right) \sim\left(\Psi(A) \cap A_{i+1}\right)$. By construction, $B_{i}$ and $\left(A \cap A_{i+1}\right)$ are disjoint, so by the Domination Lemma, $B_{i+1} \succsim C_{i+1}$. By construction, $C_{i}$ and $\left(\Psi(A) \cap A_{i+1}\right)$ are disjoint, so by the Domination Lemma, $C_{i+1} \succsim B_{i+1}$. Thus $B_{i+1} \sim C_{i+1}$ as desired.

If $I$ is finite, then $A=B_{|I|}$ and $\Psi(A)=C_{|I|}$, so $A \sim \Psi(A)$ as desired. Otherwise, $A=\lim B_{i}$ and $C=\lim C_{i}$, so by the Limit-Order Lemma twice, $A \sim \Psi(A)$ as desired.

○ Step 5: $\Psi, \Psi^{\prime}$, and $\Psi^{*}$ each preserve disjoint pairs. 
We wish to prove that for each of these mappings, the images of any disjoint pair in the domain form a disjoint pair in the co-domain. The trivial proofs for $\Psi^{\prime}$ and $\Psi^{*}$ are omitted.

Let $B, C \in \mathcal{A}$ be disjoint. Since $\left(B \cap S^{\circ}\right)=\left(\Psi(B) \cap S^{\circ}\right)$ and $\left(C \cap S^{\circ}\right)=\left(\Psi(C) \cap S^{\circ}\right)$, thus $\left(\Psi(B) \cap S^{\circ}\right)$ and $\left(\Psi(C) \cap S^{\circ}\right)$ are disjoint.

We claim for each $i \in I,\left(\Psi(B) \cap A_{i}\right)$ and $\left(\Psi(C) \cap A_{i}\right)$ are disjoint. Indeed, let $i \in I$. Assume, by way of contradiction, $\left(B \cap A_{i}\right)$ and $\left(C \cap A_{i}\right)$ are both non-null. Then since $A_{i}$ is an atom, $\left(B \cap A_{i}\right) \sim A_{i}$ and $\left(C \cap A_{i}\right) \sim A_{i} \succ \emptyset$. But then since $\left(B \cap A_{i}\right)$ and $\left(C \cap A_{i}\right)$ are disjoint, thus by the Domination Lemma $\left(B \cap A_{i}\right) \cup\left(C \cap A_{i}\right) \succ A_{i} \cup \emptyset=A_{i}$, contradicting monotonicity. Thus either $\left(B \cap A_{i}\right)$ or $\left(C \cap A_{i}\right)$ is null, so either $\Psi\left(B \cap A_{i}\right)$ or $\Psi\left(C \cap A_{i}\right)$ is empty, so $\Psi\left(B \cap A_{i}\right)$ and $\Psi\left(C \cap A_{i}\right)$ are disjoint, as desired.

Since $S=S^{\circ} \cup\left(\cup A_{i}\right)$, and since $\Psi(B)$ and $\Psi(C)$ do not intersect in $S^{\circ}$ or in any $A_{i}$, thus $\Psi(B)$ and $\Psi(C)$ are disjoint, as desired.

○ SteP 6: Verify $\left(S^{*}, \mathcal{A}^{*}, \succsim^{*}\right)$ is a catalogued qualitative probability space satisfying monotone continuity and $3^{+}-A S$.

We verify each property in sequence:

Order: To see that $\succsim^{*}$ is complete, let $A, B \in \mathcal{A}^{*}$. Since $\succsim$ is complete, $\Psi^{*}(A) \succsim$ $\Psi^{*}(B)$; thus $A \succsim^{*} B$ as desired.

To see that $\succsim^{*}$ is transitive, let $A, B, C \in \mathcal{A}^{*}$ such that $A \succsim^{*} B$ and $B \succsim^{*} C$. Then $\Psi^{*}(A) \succsim \Psi^{*}(B)$ and $\Psi^{*}(B) \succsim \Psi^{*}(C)$, so by transitivity of $\succsim, \Psi^{*}(A) \succsim \Psi^{*}(C)$; thus $A \succsim * C$ as desired.

Separability: Let $A, B, C \in \mathcal{A}^{*}$ such that $A \cap C=B \cap C=\emptyset$. Since $\Psi^{*}$ preserves disjoint pairs, $\Psi^{*}(A) \cap \Psi^{*}(C)=\Psi^{*}(B) \cap \Psi^{*}(C)=\emptyset$. Since $\succsim$ is separable, $\Psi^{*}(A) \succsim \Psi^{*}(B)$ if and only if $\Psi^{*}(A) \cup \Psi^{*}(C) \succsim \Psi^{*}(B) \cup \Psi^{*}(C)$; thus $A \succsim^{*} B$ if and only if $A \cup C \succsim^{*} B \cup C$, as desired.

Monotonicity: Let $A, B \in \mathcal{A}$ with $A \subseteq B$. Then $\Psi^{*}(A) \subseteq \Psi^{*}(B)$. Since $\succsim$ is monotonic, $\Psi^{*}(B) \succsim \Psi^{*}(A)$; thus $B \succsim^{*} A$, as desired.

Nondegeneracy: Since $\succsim$ is nondegenerate, there are $A, B \in \mathcal{A}$ such that $A \succ B$. Since $\Psi$ preserves likelihood, $\Psi(A) \succ \Psi(B)$; thus $\Psi^{\prime}(\Psi(A)) \succ^{*} \Psi^{\prime}(\Psi(B))$, as desired.

Continuity: Let $A \in \mathcal{A}^{*}$ and let $\left(B_{i}\right) \in\left(\mathcal{A}^{*}\right)^{\mathbb{N}}$ be convergent. Define $B \equiv \lim B_{i}$. Clearly, $\left(\Psi^{*}\left(B_{i}\right)\right)$ is convergent with $\Psi^{*}(B)=\lim \Psi^{*}\left(B_{i}\right)$.

If for each $i \in \mathbb{N}, B_{i} \succsim^{*} A$, then for each $i \in \mathbb{N}, \Psi^{*}\left(B_{i}\right) \succsim \Psi^{*}(A)$. Since $\succsim$ is continuous, $\Psi^{*}(B) \succsim \Psi^{*}(A)$; thus $B \succsim^{*} A$, as desired.

If for each $i \in \mathbb{N}, A \succsim^{*} B_{i}$, then by a similar argument $A \succsim^{*} B$, as desired.

$3^{+}$-AS: Let $A \in \mathcal{A}^{*}$ be an atom. Then $\Psi^{*}(A) \in \mathcal{A}$ is an atom. Since $\succsim$ satisfies $3-A S$, there are

(i) pairwise-disjoint $\mathcal{B} \subseteq\left(\downarrow \Psi^{*}(A)\right)$, and

(ii) $\mathcal{B}_{1}, \mathcal{B}_{2}, \mathcal{B}_{3} \subseteq \mathcal{B}^{\prime}$ partitioning $\mathcal{B}$

such that for each $i \in\{1,2,3\},\left(\cup \mathcal{B}_{i}\right) \succ \Psi^{*}(A)$. 
Define $\mathcal{B}^{\prime} \subseteq \mathcal{A}^{\prime}$ and $\mathcal{B}^{*} \subseteq \mathcal{A}^{*}$ by:

$$
\begin{aligned}
\mathcal{B}^{\prime} & \equiv\{\Psi(B) \mid B \in \mathcal{B}\} \\
\mathcal{B}^{*} & \equiv\left\{\Psi^{\prime}(B) \mid B \in \mathcal{B}^{\prime}\right\}
\end{aligned}
$$

Similarly, for each $i \in\{1,2,3\}$, define $\mathcal{B}_{i}^{\prime} \subseteq \mathcal{A}^{\prime}$ and $\mathcal{B}_{i}^{*} \subseteq \mathcal{A}^{*}$ by:

$$
\begin{aligned}
\mathcal{B}_{i}^{\prime} & \equiv\left\{\Psi(B) \mid B \in \mathcal{B}_{i}\right\} \\
\mathcal{B}_{i}^{*} & \equiv\left\{\Psi^{\prime}(B) \mid B \in \mathcal{B}_{i}^{\prime}\right\}
\end{aligned}
$$

Since $\Psi$ preserves likelihood and disjoint pairs,

(i) $\mathcal{B}^{\prime} \subseteq\left(\downarrow \Psi^{*}(A)\right)$,

(ii) $\mathcal{B}^{\prime}$ is pairwise-disjoint, and

(iii) $\mathcal{B}_{1}^{\prime}, \mathcal{B}_{2}^{\prime}, \mathcal{B}_{3}^{\prime} \subseteq \mathcal{B}^{\prime}$ partition $\mathcal{B}^{\prime}$

Moreover, let $i \in\{1,2,3\}$. We first claim $\mathcal{B}_{i}$ is finite or countably infinite. Indeed, there is no non-decreasing sequence $\left(B_{j}\right) \in\left(\mathcal{B}_{i}\right)^{\mathbb{N}}$; else since $\mathcal{B}_{i}$ is pairwise-disjoint, $\emptyset=\lim B_{j}$, so by continuity $\emptyset \succsim B_{1}$, contradicting $B_{1} \in\left(\downarrow \Psi^{*}(A)\right)$. If $\mathcal{B}_{i}$ is not finite, then we can construct $\left(B_{j}\right) \in\left(\mathcal{B}_{i}^{\prime}\right)^{\mathbb{N}}$ by iteratively taking the most-likely remaining event as in Step 2, and since $\mathcal{B}_{i}$ is pairwise-disjoint, $\emptyset=\lim B_{j}$. In this case, $\left\{B_{j}\right\}=\mathcal{B}_{i}$; else there is $B \in \mathcal{B}_{i} \backslash\left\{B_{j}\right\}$, and by construction, for each $j \in \mathbb{N}, B_{j} \succsim B$, so by continuity $\emptyset=\lim B_{j} \succsim B$, contradicting $B \in\left(\downarrow \Psi^{*}(A)\right)$. Thus $\mathcal{B}_{i}$ is finite or countably infinite, as desired.

Label the members of $\mathcal{B}_{i}$ by $\left\{B_{1}, B_{2}, \ldots, B_{j}, \ldots\right\}=\left\{B_{j}\right\}_{j \in J}$, where $J \subseteq \mathbb{N}$ may or may not be finite. For each $j \in J$, define $B_{j}^{\prime} \equiv \Psi\left(B_{j}\right)$, define $C_{j} \equiv \cup_{k \leq j} B_{k}$, and define $C_{j}^{\prime} \equiv \cup_{k \leq j} \Psi\left(B_{k}\right)$.

We claim that for each $j \in J, C_{j} \sim C_{j}^{\prime}$, which we prove by induction through an argument similar to that in Step 4. For the base step, since $\Psi$ preserves likelihood, $C_{1} \sim C_{1}^{\prime}$. For the inductive step, assume that $C_{j} \sim C_{j}^{\prime}$. Since $\Psi$ preserves likelihood, $B_{j+1} \sim B_{j+1}^{\prime}$. By construction, $C_{j}$ and $B_{j+1}$ are disjoint, so by the Domination Lemma, $C_{j+1} \succsim C_{j+1}^{\prime}$. By construction, $C_{j}^{\prime}$ and $B_{j+1}^{\prime}$ are disjoint, so by the Domination Lemma, $C_{j+1}^{\prime} \succsim C_{j+1}$. Thus $C_{j+1} \sim C_{j+1}^{\prime}$, as desired.

If $J$ is finite, then $\cup \mathcal{B}_{i}=C_{|J|}$ and $\cup \mathcal{B}_{i}^{\prime}=C_{|J|}^{\prime}$, so $\cup \mathcal{B}_{i} \sim \cup \mathcal{B}_{i}^{\prime}$. Otherwise, $\mathcal{B}_{i}=\lim C_{j}$ and $\mathcal{B}_{i}^{\prime}=\lim C_{j}^{\prime}$, so by the Limit-Order Lemma twice, $\cup \mathcal{B}_{i} \sim \cup \mathcal{B}_{i}^{\prime}$. Thus $\cup \mathcal{B}_{i}^{\prime} \succ \Psi^{*}(A)$.

By definition of $\Psi^{\prime}$, since $\Psi^{\prime}$ preserves disjoint pairs, and since $\Psi^{\prime}$ is the inverse of $\Psi^{*}$, it follows from the above properties of $\mathcal{B}^{\prime}, \mathcal{B}_{1}^{\prime}, \mathcal{B}_{2}^{\prime}, \mathcal{B}_{3}^{\prime}$ that:

(i) $\mathcal{B}^{*} \subseteq(\downarrow A)$,

(ii) $\mathcal{B}^{*}$ is pairwise-disjoint,

(iii) $\mathcal{B}_{1}^{*}, \mathcal{B}_{2}^{*}, \mathcal{B}_{3}^{*} \subseteq \mathcal{B}^{\prime}$ partition $\mathcal{B}^{*}$, and

(iv) for each $i \in\{1,2,3\}, \cup \mathcal{B}_{i}^{*} \succ^{*} A$, as desired.

Catalogued: To see that $\left(S^{*}, \mathcal{A}^{*}, \succsim^{*}\right)$ is catalogued, simply take $S^{\oplus}=I$.

○ STEP 7: $\left(S^{*}, \mathcal{A}^{*}, \succsim^{*}\right)$ has representation $\mu^{*} \in \mathbb{M}^{\sigma}\left(\mathcal{A}^{*}\right)$ with the desired properties.

Since $\left(S^{*}, \mathcal{A}^{*}, \succsim^{*}\right)$ is a catalogued qualitative probability space satisfying continuity and $3^{+}-A S$, this is immediate from Theorem 2 and Theorem 4 .

○ Step 8: $(S, \mathcal{A}, \succsim)$ has unique representation $\mu \in \mathbb{M}^{\sigma}(\mathcal{A})$ with the desired properties. 
For each $A \in \mathcal{A}^{*}$, define $\mu\left(\Psi^{*}(A)\right) \equiv \mu^{*}(A)$. For each $i \in I$ and each $B \subseteq A_{i}$, define $\mu(B) \equiv \mu\left(A_{i}\right)$ if $B \sim A_{i}$ and define $\mu(B) \equiv 0$ otherwise. It is straightforward to complete the definition of $\mu$ to be a $\sigma$-measure and to verify its properties.

\section{References}

Abdellaoui, M. And Wakker, P. (2005). "The Likelihood Method for Decision under Uncertainty." Theory and Decisions 58, 3-76.

Adams, E. (1962). "On Rational Betting Systems." Archiv für Mathematische Logik und Grundlagenforschung $6,7-18$ and 112-128.

Allais, M. (1953). "Le Comportement de l'Homme Rationnel devant le Risque, Critique des Postulats et Axiomes de l'Ecole Américaine." Econometrica 21, 503-546.

Allais, M. (1979). "The So-Called Allais Paradox and Rational Decisions Under Uncertainty." In: Expected Utility Hypotheses and the Allais Paradox. Editors: Allais, M. and Hagen, O. Dodrecht, the Netherlands: D. Reidel Publishing Company.

Alon, S. AND Lehrer, E. (2014). "Subjective multi-prior probability: A representation of a partial likelihood relation." Journal of Economic Theory 151, 476-492.

Anscombe, F., Aumann, R. (1963). "A definition of subjective probability." Annals of Mathematical Statistics 34, 199-205.

Aumann, R. (1962). "Utility theory without the completeness axiom." Econometrica 30, 445-462.

Arrow, K. (1970). Essays in the Theory of Risk-Bearing. Chicago, IL: Markham.

Banach, S. (1932). Théorie des opérations linéaires [in French]. Warsaw, Poland: Fundusz Kultury Narodowej.

Banach, S. and Kuratowski, C. (1929). "Sur une généralisation du problème de la mesure [in French]." Fundamenta Mathematicae 14, 127-131.

Barbanel, J. And TAYlor, A. (1995). "Preference relations and measures in the context of fair division." Proceedings of the American Mathematical Society 123, 2061-2070.

BAyes, T. (1763). "An Essay toward solving a Problem in the Doctrine of Chances." Communicated by Price, R. Philosophical Transactions of the Royal Society of London 53, 370-418.

Berliant, M. (1986). "A utility representation for a preference relation on a $\sigma$-algebra." Econometrica $54,359-362$.

Berliant, M. (1985). "Equilibrium Models with Land: A Criticism and an Alternative." Regional Science and Urban Economics 15, 325-340.

Berliant, M., Thomson, W., And Dunz, K. (1992). "On the fair division of a heterogeneous commodity." Journal of Mathematical Economics 21, 201-206.

Bernstein, S. (1917). "Attempt at an axiomatic foundation of probability theory [in Russian]." Communications of the Kharkov Mathematical Society [in Russian] 15, 209-274. Translation In: Sheynin, O. (2005). Probability and Statistics: Russian Papers of the Soviet Period. Berlin, Germany: NG Verlag.

Birkhoff, G. (1935). "On the structure of abstract algebras." Proceedings of the Cambridge Philosophical Society 31, 433-454.

Birkhoff, G. (1937). "Rings of sets." Duke Mathematical Journal 3, 443-454. 
Bleichrodt, H., Rohde, K., And Wakker, P. (2008). "Koopmans' constant discounting for intertemporal choice: A simplification and a generalization." Journal of Mathematical Psychology 52, 341-347.

Boole, G. (1851). "On the Theory of Probabilities, and in particular on Mitchell's Problem of the Distribution of the Fixed Stars." The London, Edinburgh, and Dublin Philosophical Magazine and Journal of Science Supplement to Series 4 Volume 1, 521-530.

Boole, G. (1854). "Reply to some Observations published by $M r$. Wilbraham in the Philosophical Magazine, vol. vii. p. 465, on the Theory of Chances developed in Professor Boole's 'Laws of Thought."' The London, Edinburgh, and Dublin Philosophical Magazine and Journal of Science, Series 4 Volume 8, $87-91$.

Buskes, G., De Pagter, B., And van RooiJ, A. (2008). "The Loomis-Sikorski Theorem revisited." Algebra universalis 58, 413-426.

CAntor, G. (1883). "Über unendliche, lineare Punktmannigfaltigkeiten V [in German]." Mathematische Annalen 21, 545-591.

Cantor, G. (1895). "Beiträge zur Begründung der transfiniten Mengenlehre [in German]." Mathematische Annalen 46, 481-512.

Cettolin, E. And Riedl, A. (2013). "Revealed Incomplete Preferences under Uncertainty." Working paper.

Chateauneuf, A. (1985). "On the Existence of a Probability Measure Compatible with a Total Preorder on a Boolean Algebra." Journal of Mathematical Economics 14, 43-52.

Chateauneuf, A. And Jaffray, J. (1984). "Archimedean qualitative probabilities." Journal of Mathematical Psychology 28, 191-204.

Chateauneuf, A., Maccheroni, F., Marinacci, M., and Tallon, J. (2005). "Monotone continuous multiple priors." Economic Theory 26, 973-982.

Chew, S. And Sagi, J. (2006). "Event exchangeability: probabilistic sophistication without continuity or monotonicity." Econometrica 74, 771-786.

Debreu, G. (1954). "Representation of a preference ordering by a numerical utility function." In: Decision Processes.. Editors: Thrall, R. Coombs, C., and Davis, R. San Francisco, CA: John Wiley \& Sons.

Debreu, G. (1959). "Topological Methods in Cardinal Utility Theory." In: Mathematical Methods in the Social Sciences. Editors: Arrow, K., Karlin, S., and Suppes, P. Stanford, CA: Stanford University Press.

Debreu, G. (1964). "Continuity Properties of Paretian Utility." International Economic Review 5, 285293.

Domotor, Z. (1969). "Probabilistic relational structures and their applications." Technical Report Number 144, Institute for Mathematical Studies in the Social Sciences. Standard, CA: Stanford University.

EliAZ, K. AND OK, E. (2006). "Indifference or indecisiveness? Choice-theoretic foundations of incomplete preferences." Games and Economic Behavior 56, 61-86.

Ellsberg, D. (1961). "Risk, Ambiguity, and the Savage Axioms." The Quarterly Journal of Economics 75, 643-669.

Epstein, L. And Zhang, J. (2001). "Subjective probabilities on subjectively unambiguous events." Econometrica 69, 265-306. 
Ergin, H. And Gul, F. (2009). "A theory of subjective compound lotteries." Journal of Economic Theory 144, 899-929.

EuClid. (circa 300 BC). Elements. Translation: Heath, T. (1908). The thirteen books of Euclid's Elements. Cambridge, United Kingdom: Cambridge University Press.

DE Finetri, B. (1937). "La prévision: ses lois logiques, ses sources subjectives [in French]." Annales d'Institut Henri Poincaré 7, 1-68.

FARIS, W. (2007). Real Analysis Structures. Manuscript.

Fishburn, P. (1970). Utility Theory for Decision Making. New York, NY: John Wiley \& Sons, Inc.

Fishburn, P. (1986). "The Axioms of Subjective Probability." Statistical Science 1, 335-358.

Garey, M. And Johnson, D. (1979). Computers and Intractibility: A Guide to the Theory of NPCompleteness. New York, NY: W.H. Freeman and Company.

Gul, F. (1992). "Savage's Theorem with a Finite Number of States." Journal of Economic Theory 57, 99-110.

GilboA, I. And Schmeidler, D. (1989). "Maxmin expected utility with a non-unique prior." Journal of Mathematical Economics 18, 141-153.

Halmos, P. (1947). "On the set of values of a finite measure." Bulletin of the American Mathematical Society 53, 138-141.

Halmos, P. (1963). Lectures on Boolean Algebras. New York, NY: Van Nostrand.

Halpern, J. And LÉvy, A. (1964). "The Boolean prime ideal theorem does not imply the axiom of choice." Proceedings of the Symposium in Pure Mathematics of the American Mathematical Society 13, 83-134.

HilberT, D. (1900). "Mathematische probleme [in German]." Nachrichten von der Gesellschaft der Wissenschaften zu Göttingen, Mathematisch-Physikalische Klasse Volume 1900, 253-297. Translation: Hilbert, D. (1902). "Mathematical Problems." Translator: Mary Winston Newson. Bulletin of the American Mathematical Society 8, 437-479.

KAKEyA, S. (1914). "On the set of partial sums of an infinite series." Proceedings of the Tokyo Mathematico-Physical Society 7, 250-251.

KAKEYA, S. (1915). "On the partial sums of an infinite series." Science reports of the Tôhoku Imperial University 3, 159-163.

Knight, F. (1921). Risk, Uncertainty, and Profit. Boston, Massachusetts: Houghton Mifflin Company.

Kochov, A. (2013). "Geometric Discounting in Discrete, Infinite-Horizon Choice Problems." Working paper.

Kolmogoroff, A. (1933). Grundbegriffe der Wahrscheinlichkeitsrechnung [in German]. Berlin, Germany: Julius Springer. Translation: Kolmogorov, A. (1956). Foundations of the Theory of Probability, Second English Edition. Translator: Nathan Morrison. New York, New York: Chelsea Publishing Company.

Kolmogorov, A. (1948). "Obituary: Evgeny Evgenievich Slutsky (in Russian)." Translation: (2002). Translator: Oscar Sheynin. The Mathematical Scientist 27, 67-74.

Komornik, V. (2015). Private communication.

Koopman, B. (1940). "The axioms and algebra of intuitive probability." Annals of Mathematics 41, 269-292. 
Koopmans, T. (1960). "Stationary Ordinal Utility and Impatience." Econometrica 28, 287-309.

Koopmans, T. (1972). "Representations of preference orderings with independent components of consumption, and representations of preference orderings over time." In: Decision and Organization: a volume in honor of Jacob Marschak. Editors: McGuire, C. and Radner, R. Amsterdam, the Netherlands: North-Holland Publishing Company.

Kopylov, I. (2007). "Subjective probabilities on 'small' domains." Journal of Economic Theory 113, 236-265.

Kopylov, I. (2010). "Simple axioms for countably additive subjective probability." Journal of Mathematical Economics 46, 867-876.

Kraft, C., Pratt, J., And Seidenberg, A. (1959). "Intuitive Probability on Finite Sets." Annals of Mathematical Statistics 30, 408-419.

Krantz, D., Luce, R., Suppes, P., And Tversky, A. (1971). Foundations of Measurement Volume I: Additive and Polynomial Representations. New York, NY: Academic Press.

Kreps, D. (1988). Notes on the theory of choice. Boulder, CO: Westview Press, Inc.

Laibson, D. (1997). "Golden Eggs and Hyperbolic Discounting." The Quarterly Journal of Economics 112, 443-477.

Loewenstein, G. and Prelec, D. (1992). "Anomalies in intertemporal choice: Evidence and an interpretation." The Quarterly Journal of Economics 107, 573-597.

Loomis, L. (1947). "On the representation of $\sigma$-complete Boolean algebras." Bulletin of the American Mathematical Society 53, 757-760.

Machina, M., Schmeidler, D. (1992). "A More Robust Definition of Subjective Probability." Econometrica $60,745-780$.

Mackey, G. (1957). "Borel structures in groups and their duals." Transactions of the American Mathematical Society 85, 134-165.

Mandler, M. (2005). "Incomplete preferences and rational intransitivity of choice." Games and Economic Behavior 50, 255-277.

MARINACCI, M. (1993). "On the Ranges of Baire and Borel Measures, with Applications to Fine and Tight Comparative Probabilities." Manuscript.

NeHring, K. (2009). "Imprecise probabilistic beliefs as a context for decision-making under ambiguity." Journal of Economic Theory 144, 1054-1091.

von Neumann, J., Morgenstern, O. (1947). The Theory of Games and Economic Behavior. Princeton, NJ: Princeton University Press.

Nunke, R. and Savage, L. (1952). "On the set of values of a nonatomic, finitely additive, finite measure." Proceedings of the American Mathematical Society 3, 217-218.

Olea, J. and Strzalecki, T. (2014). "Axiomatization and measurement of Quasi-hyperbolic Discounting." Quarterly Journal of Economics 129, 1449-1499.

Peano, G. (1889). Arithmetices principia: nova methodo. Rome, Italy: Fratres Bocca.

Ramsey, F. (1931). "Truth and Probability." In: Foundations of Mathematics and other Logical Essays. Editor: Braithwaite, R. London: Kegan Paul, Trench, Trubner, and Co., Ltd.

RÉNYI, A. (1957). "Representations for real numbers and their ergodic properties." Acta Mathematica Hungarica 8, 477-493. 
Samuelson, P. (1937). "A Note on Measurement of Utility." The Review of Economic Studies 4, 155161.

Savage, L. (1954). The Foundations of Statistics. Wiley, NY: Dover Publications.

Savage, L. (1961). "The Foundations of Statistics Reconsidered." In: Proceedings of the Fourth Berkeley Symposium of Mathematical Statistics and Probability, Volume 1: Contributions to the Theory of Statistics. Berkeley, California: University of California Press.

Savage, L. (1972). The Foundations of Statistics: Second Revised Edition. Wiley, NY: Dover Publications.

Scott, D. (1964). "Measurement Models and Linear Inequalities." Journal of Mathematical Psychology $1,233-247$.

Schmeidler, D. (1989). "Subjective Probability and Expected Utility without Additivity." Econometrica $57,571-587$.

Seidenfeld, T. And Schervish, M. (1983). "A Conflict Between Finite Additivity and Avoiding Dutch Books." Philosophy of Science 50, 398-412.

Sheynin, O. (2005). Probability and Statistics: Russian Papers of the Soviet Period. Berlin, Germany: NG Verlag.

Sheynin, O. (2010). Evgeny Slutsky: Collected Statistical Papers. Berlin, Germany: NG Verlag.

Sikorski, R. (1960). Boolean Algebras. Berlin, Germany: Springer-Verlag.

Slutsky, E. (1922). "On the Logical Foundation of the Calculus of Probability (in Russian)." Vestnik Statistiki 12, 13-21.

Slutsky, E. (1925). "On the Law of Large Numbers (in Russian)." Vestnik Statistiki 22, 1-55.

Steinhaus, H. (1948). "The problem of fair division." Econometrica 16, 101-104.

Stinchcombe, M. (1997). "Countably Additive Subjective Probabilities." The Review of Economic Studies 64, 125-146.

Stone, M. (1936). "The Theory of Representations of Boolean Algebras." Transactions of the American Mathematical Society 40, 37-111.

Ulam, S. (1930). "Zur Masstheorie in der allgemeinen Mengenlehre." Fundamenta Mathematicae 16, $140-150$

Villegas, C. (1964). "On Quantitative Probability $\sigma$-Algebras." Annals of Mathematical Statistics 35, $1787-1796$.

Villegas, C. (1967). "On Qualitative Probability." The American Mathematical Monthly 74, 661-669.

DE VRies, M. ANd Komornik, V. (2009). "Unique expansions of real numbers." Advances in Mathematics 221, 390-427.

Wakker, P. (1981). "Agreeing Probability Measures for Comparative Probability Structures." The Annals of Statistics 9, 658-662.

Wakker, P. (1989). Additive Representations of Preferences, A New Foundation of Decision Analysis. Dodrecht, the Netherlands: Kluwer Academic Publishers.

WAKKer, P. (1993a). "Clarification of some mathematical misunderstandings about Savage's foundations of statistics, 1954." Mathematical Social Sciences 25, 199-202.

WAKker, P. (1993b). "Savage's Axioms Usually Imply Violation of Strict Stochastic Dominance." The Review of Economic Studies 60, 487-493. 